PSI-1454/

TR-2211

\title{
High Altitude Aerial Natural Gas Leak Detection System
}

\author{
Program Final Report \\ Covering the Period \\ October 2004 through December 2006
}

\author{
Richard T. Wainner, Mickey B. Frish, B. David Green, \\ Matthew C. Laderer, Mark G. Allen, and Joseph R. Morency
}

April 2007

Contract No. DE-FC26-04NT42268

\author{
Submitted by: \\ Physical Sciences Inc. \\ 20 New England Business Center \\ Andover, MA 01810-1077 \\ Submitted to: \\ Department of Energy \\ National Energy Technology Laboratory \\ 626 Cochrans Mill Road \\ P.O. Box 10940 \\ Pittsburgh, PA 15236-0940
}

This Program Final Report was prepared with the support of the U.S. Department of Energy, under Award No. DE-FC2604NT42268. However, any opinions, findings, conclusions, or recommendations expressed herein are those of the author(s) and do not necessarily reflect the view of the DOE. 



\section{DISCLAIMER}

This report was prepared as an account of work sponsored by an agency of the United States Government. Neither the United States Government nor any agency thereof, nor any of their employees, makes any warranty, express or implied, or assumes any legal liability or responsibility for the accuracy, completeness, or usefulness of any information, apparatus, product, or process disclosed, or represents that its use would not infringe privately owned rights. Reference herein to any specific commercial product, process, or service by trade name, trademark, manufacturer, or otherwise does not necessarily constitute or imply its endorsement, recommendation, or favoring by the United States Government or any agency thereof. The views and opinions of authors expressed herein do not necessarily state or reflect those of the United States Government or any agency thereof. 



\begin{abstract}
The objective of this program was to develop and demonstrate a cost-effective and power-efficient advanced standoff sensing technology able to detect and quantify, from a highaltitude $(>10,000 \mathrm{ft})$ aircraft, natural gas leaking from a high-pressure pipeline. The advanced technology is based on an enhanced version of the Remote Methane Leak Detector (RMLD) platform developed previously by Physical Sciences Inc. (PSI). The RMLD combines a telecommunications-style diode laser, fiber-optic components, and low-cost DSP electronics with the well-understood principles of Wavelength Modulation Spectroscopy (WMS), to indicate the presence of natural gas located between the operator and a topographic target. The transceiver transmits a laser beam onto a topographic target and receives some of the laser light reflected by the target. The controller processes the received light signal to deduce the amount of methane in the laser's path. For use in the airborne platform, we modified three aspects of the RMLD, by: 1) inserting an Erbium-doped optical fiber laser amplifier to increase the transmitted laser power from $10 \mathrm{~mW}$ to $5 \mathrm{~W} ; 2$ ) increasing the optical receiver diameter from $10 \mathrm{~cm}$ to 25 $\mathrm{cm}$; and 3) altering the laser wavelength from $1653 \mathrm{~nm}$ to $1618 \mathrm{~nm}$. The modified RMLD system provides a path-integrated methane concentration sensitivity $\sim 5000 \mathrm{ppm}-\mathrm{m}$, sufficient to detect the presence of a leak from a high capacity transmission line while discriminating against attenuation by ambient methane. In ground-based simulations of the aerial leak detection scenario, we demonstrated the ability to measure methane leaks within the laser beam path when it illuminates a topographic target $2000 \mathrm{~m}$ away. We also demonstrated simulated leak detection from ranges of $200 \mathrm{~m}$ using the $25 \mathrm{~cm}$ optical receiver without the fiber amplifier.
\end{abstract}




\section{TABLE OF CONTENTS}

$\underline{\text { Section }}$

Page

1 .

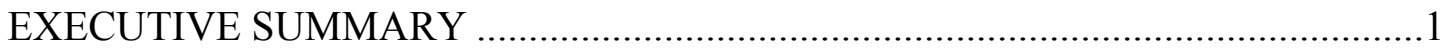

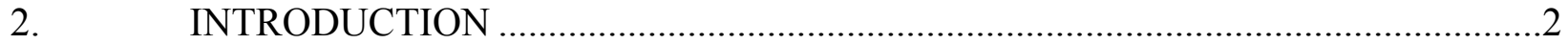

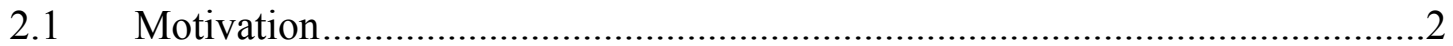

2.2 Technical Approach ..............................................................................2

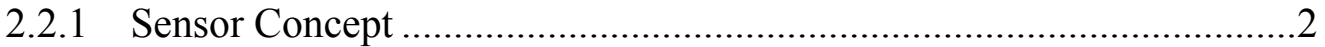

2.2.2 Measurement Details ..................................................................

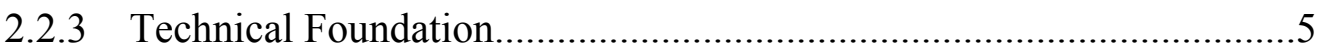

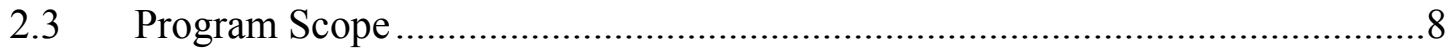

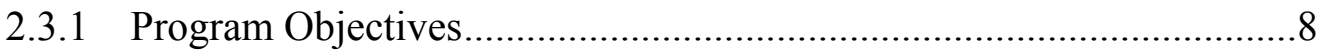

2.3.2 Program Tasks .............................................................................

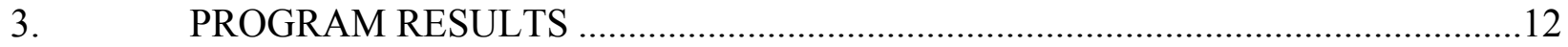

3.1 Program Results Summary .....................................................................12

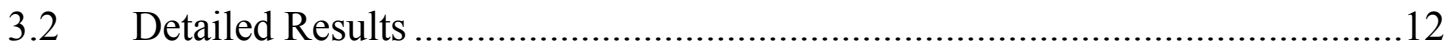

3.2.1 Task 1: Research Management Plan ......................................................12

3.2.2 Task 2: Technology Status Assessment ……………………...............13

3.2.3 Task 3: System Architecture and Specifications ..................................13

3.2.4 Task 4: System Design, Acquisition, and Assembly ...........................13

3.2.5 Task 5: Laboratory and Outdoor Ground Testing................................20

3.2.6 Task 6: Flight Test Preparation.............................................................45

3.2.7 Task 7: Simulated Flight Test...........................................................49

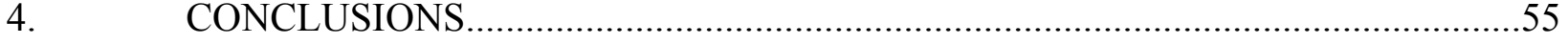

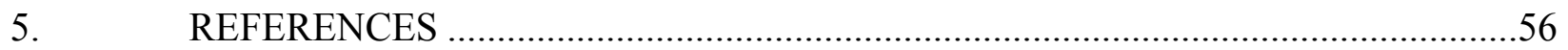
APPENDICES

A. Research Management Plan .....................................................................57

B. Technology Status Assessment.......................................................................67

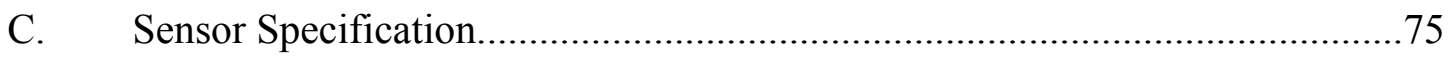

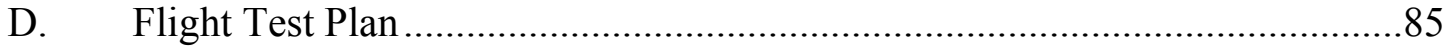




\section{LIST OF FIGURES}

Figure No.

$\underline{\text { Page }}$

1. Photographs of PSI's remote methane leak detector (RMLD) production version (left) and advanced prototype during field testing (right) 3

2. Schematic of airborne amplified RMLD sensor concept..........................................4

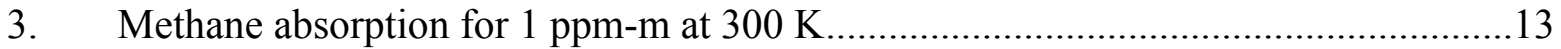

4. Detailed examination of atmospheric absorption at $300 \mathrm{~K}, 6100 \mathrm{~m}$ pathlength...........14

5. Modeled signal/noise ratio for a $1000 \mathrm{ppm}-\mathrm{m} \mathrm{CH}_{4}$ plume versus sensor rangeto-target.

6. EDFA Output Spectrum................................................................................... 16

7. Gamm Air's Cessna 207, cabin with photographic equipment installed, and exterior view of camera port

8. Celestron C10-N telescope (left) and Edmund Scientific Model NT39-244

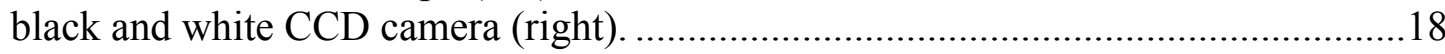

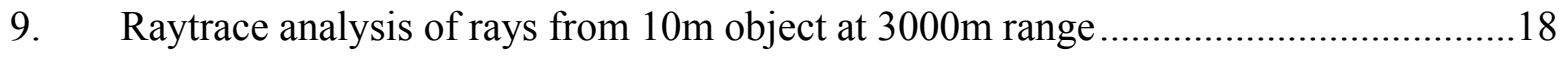

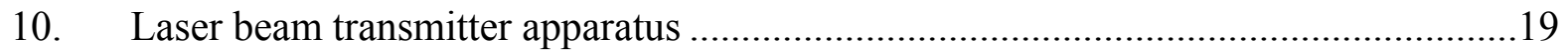

11. Raytrace images at the detector plane (and related collection efficiencies and focal plane locations) for various sensor-to-target ranges .....................................19

12. Airborne RMLD system mounting configuration.............................................20

13. Schematic of laboratory benchtop hardware for airborne RMLD component characterization

14. WMS concentration signal for signals received with the unamplified laser and benchtop transceiver from a door at $5 \mathrm{~m}$ distance, with (red)and without (green) an $\sim 10 \mathrm{~cm}$ thick bag of pure methane in the beam path

15. Seed laser power transmitted through $50 \mathrm{~cm}$ cell with (blue) no gas, and (red) 75 torr neat methane.

16. HITRAN-calculated methane spectral feature selected for the high-altitude leak detector at various pressures

17. Measured absorbance of seed laser power versus time for 75 torr neat methane in $50 \mathrm{~cm}$ cell 


\section{LIST OF FIGURES (Continued)}

Figure No. $\quad \underline{\text { Page }}$

18. Measured absorbance versus time for 760 torr neat methane in a $50 \mathrm{~cm}$ cell .............23

19. Measured signal from the "seed" laser $(10 \mathrm{kHz}, 14 \mathrm{~mA}$ modulation) through a $2 \mathrm{GHz}$ etalon (left) and the resultant calculation of wavelength modulation

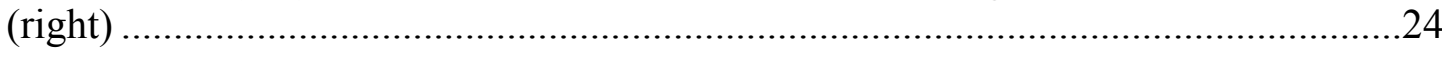

20. 1f, $2 \mathrm{f}$, and concentration, measured using the seed laser in a $50 \mathrm{~cm}$ cell .......................24

21. Amplifier output power versus pump laser current settings for fixed seed laser input power of $18.6 \mathrm{~mW}$......................................................................................25

22. Laser amplifier output versus pump laser settings during modulated seed laser input

23. Ratio of modulation amplitude to average power for amplified laser, compared to same ratio for seed laser, as function of amplifier gain

24. Temporal variation of $2 \mathrm{f} / 1 \mathrm{f}$ resulting from amplitude modulation distortion in the fiber amplifier

25. Portion of amplified laser power transmitted through $50 \mathrm{~cm}$ cell with (blue) no gas, and (red) 75 torr neat methane.

26. (green) Absorbance of 75 torr neat methane, calculated from data of Figure 25 ........29

27. Measured absorbance, using amplified laser, of 760 torr of (red) neat methane and (blue) $5 \%$ methane in $50 \mathrm{~cm}$ cell at 760 torr

28. 1f, $2 \mathrm{f}$, and concentration measured using the amplified laser......................................30

29. Tripod-mounted transceiver and electronics in box truck ...........................................31

30. Absorbance generated using the telescope as a calibration cell with $10 \% \mathrm{CH}_{4}$ in $\mathrm{N}_{2}$, for both amplified and unamplified lasers at $\sim 5 \mathrm{~m}$ target range..........................31

31. Camera view of plywood target with retroreflective tape at $200 \mathrm{~m}$ range....................32

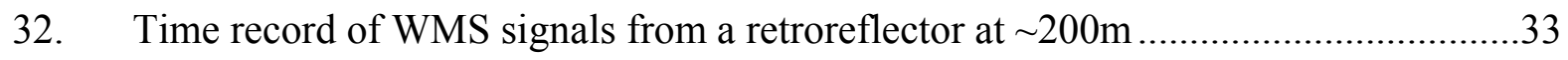

33. (left) Signals.recorded directly from detector/preamp combo (retroreflector target), including (black) before the $10 \% \mathrm{CH}_{4}$ calibration gas is turned on and (red) at a point when the telescope is nearly filled with the gas $(208,000 \mathrm{ppm}-$ m). (right) $\mathrm{CH}_{4}$ absorbance calculated from the two recorded signals. 


\section{LIST OF FIGURES (Continued)}

Figure No.

$\underline{\text { Page }}$

34. (left) Signals.recorded directly from detector/preamp combo (retroreflector target), including (black) before the $10 \% \mathrm{CH}_{4}$ calibration gas is turned on and (red) at a point when the telescope is partially filled with the gas (133,000ppm-m). (right) $\mathrm{CH}_{4}$ absorbance calculated from the two recorded signals

35. Time record of WMS signals from a plywood target at $\sim 200 \mathrm{~m}$ using the EDFA-amplified laser. At $\sim 180 \mathrm{sec}, 10 \% \mathrm{CH}_{4}$ (balance $\mathrm{N}_{2}$ ) begins flowing into the telescope

36. (left) Signals.recorded directly from detector/preamp combo (plywood target) using the EDFA-amplified laser, including (black) before the $10 \% \mathrm{CH}_{4}$ calibration gas is turned on and (red) at a point when the telescope is at an equilibrium concentration of calibration gas (222,000ppm-m). (right) $\mathrm{CH}_{4}$ absorbance calculated from the two recorded signals.

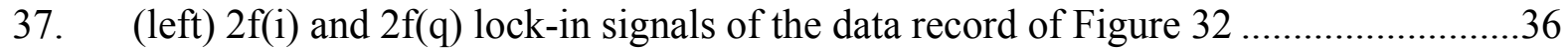

38. Time record of WMS signals from a plywood target at $\sim 200 \mathrm{~m}$ using the EDFA-amplified laser.

39. Water tower as viewed by transceiver video camera .38

40. Record of 1 f signals logged while performing vertical scans across the water tower

41. Time record of WMS signals from a water tower at $\sim 2000 \mathrm{~m}$ using the EDFAamplified laser

42. Time record of WMS $2 \mathrm{f}$ quadrature component signals from a water tower at $\sim 2000 \mathrm{~m}$ using the EDFA-amplified laser

43. Benchtop experimental setup for laboratory EDFA distortion analysis

44. 65-hour time record of WMS signals from a beam dump employing the EDFA-amplified laser.

45. Seed layer (black) and amplified laser (red) power waveforms at modulation frequencies from 20 to $100,000 \mathrm{~Hz}$......

46. WMS signals recorded at $100 \mathrm{kHz}$ modulation frequency

47. Output signal (power) from the EDFA versus wavelength via injection current (left) and temperature (right) laser tuning. 


\section{LIST OF FIGURES (Continued)}

Figure No. $\quad \underline{\text { Page }}$

48. Aerial view of PSI facility and employed sensor-to-target paths ...............................46

49. View from sensor transceiver to measurement location for the $115 \mathrm{~m}$ path (left) and $185 \mathrm{~m}$ path (right).....................................................................................47

50. Methane target assembly mounted to the roof of an automobile..................................48

51. Recorded camera images from simulated flight test experiments at $115 \mathrm{~m}$ range (left) and 185m range (right).........................................................................48

52. Graphical user interface employed in simulated flight test experiments

53. $10 \mathrm{~Hz}$ data records of $\mathrm{CH}_{4}$ concentration signals from the airborne remote methane leak detector (aRMLD) detecting a calibrated 11,500ppm-m gas sample passing through its field of view at $115 \mathrm{~m}$ range at three different speeds.

54. Calculated detection limits for the airborne remote methane leak detector (aRMLD) versus target speed, based on the SNR of data records such as in Figure 53

55. Zoom of $10 \mathrm{~Hz}$ data record of $\mathrm{CH}_{4}$ concentration signals from the airborne remote methane leak detector (aRMLD) detecting a calibrated 11,500ppm-m gas sample passing at $10 \mathrm{mph}$

56. Recorded maximum (red) and rms noise signals (with (yellow) and without (green) noise spikes included) for the airborne remote methane leak detector (aRMLD) versus target speed

57. Calculated detection limits for the airborne remote methane leak detector (aRMLD) versus target speed, based on the SNR of data records such as in Figure 7, with noise spikes removed.

58. $10 \mathrm{~Hz}$ data records of $\mathrm{CH}_{4}$ concentration signals from the airborne remote methane leak detector (aRMLD) detecting a calibrated 10,000ppm-m gas sample at a $185 \mathrm{~m}$ range.

59. $10 \mathrm{~Hz}$ data records of $\mathrm{CH}_{4}$ concentration signals from the airborne remote methane leak detector (aRMLD) detecting a calibrated 10,000ppm-m gas sample passing through its field of view at a $185 \mathrm{~m}$ range at three different speeds. 


\section{EXECUTIVE SUMMARY}

The objective of this program was to develop and demonstrate a cost-effective and power-efficient advanced standoff sensing technology able to detect and quantify, from a highaltitude (> 10,000 ft) aircraft, natural gas leaking from a high-pressure pipeline. The advanced technology is based on an enhanced version of the Remote Methane Leak Detector (RMLD) platform developed previously by Physical Sciences Inc. (PSI). The RMLD combines a telecommunications-style diode laser, fiber-optic components, and low-cost DSP electronics with the well-understood principles of Wavelength Modulation Spectroscopy (WMS), to indicate the presence of natural gas located between the operator and a topographic target. The system includes an optical transceiver and an electronic controller. The transceiver transmits a laser beam onto a topographic target and receives some of the laser light reflected by the target. The controller processes the received light signal to deduce the amount of methane in the laser's path.

The currently-available lightweight, handheld, battery-powered RMLD provides a maximum range to the topographic target of $100 \mathrm{ft}$. For use in the airborne platform, we modified three aspects of the RMLD, by: 1) inserting an Erbium-doped optical fiber laser amplifier to increase the transmitted laser power from $10 \mathrm{~mW}$ to $5 \mathrm{~W} ; 2$ ) increasing the optical receiver diameter from $10 \mathrm{~cm}$ to $25 \mathrm{~cm}$; and 3) altering the laser wavelength from $1653 \mathrm{~nm}$ to $1618 \mathrm{~nm}$. The modified RMLD system provides a path-integrated methane concentration sensitivity $\sim 5000 \mathrm{ppm}-\mathrm{m}$, sufficient to detect the presence of a leak from a high capacity transmission line while discriminating against attenuation by ambient methane.

A significant portion of the project was devoted to characterizing the performance of the amplified TDLAS system. To our knowledge, this was the first time that Erbium-doped fiber amplifier (EDFA) has been utilized in conjunction with Wavelength Modulation Spectroscopy. We learned that, at the conditions where we operated the system, the characteristics of the amplifier interact with the laser modulation to degrade the measurement sensitivity. In particular, amplifier thermal drifts create an offset in the methane measurement that varies over time, which limits the absolute accuracy of the methane measurement. However, for the leak detection application, we compensate for this slow thermal drift by activating leak alarms based on relatively rapid changes in measured methane.

Although the original project plan envisioned a flight test of the system, we were unable by the end of the project to secure an acceptable test site. Instead, we performed several groundbased simulations of the aerial leak detection scenario. In these simulations, we demonstrated the ability to measure methane leaks within the laser beam path when it illuminates a topographic target $2000 \mathrm{~m}$ away. We also demonstrated simulated leak detection from ranges of $200 \mathrm{~m}$ using the $25 \mathrm{~cm}$ optical receiver without the fiber amplifier. This configuration enables aerial survey at altitudes up to approximately $750 \mathrm{~m}$ with detection limits $\leq 200 \mathrm{ppm}-\mathrm{m}$. 


\section{INTRODUCTION}

\section{$2.1 \quad$ Motivation}

The US natural gas transmission system comprises approximately 250,000 miles of pipeline, 1700 transmission stations and 17,000 compressors. This transmission system serves local distribution companies that operate some 500-1000 gate stations supplying roughly 132,000 surface metering and pressure regulation sites stationed along 1,000,000 miles of distribution pipeline terminating at 61,000,000 end-user customer meters. Maintaining the security and integrity of this system is a continual process of searching for, locating, and repairing leaks.

Leak surveying is very labor intensive, in part because all currently available natural gas detectors must be positioned within a leak plume to detect the leak. In relation to this challenge, and prior to this program, Physical Sciences Inc. (PSI), in conjunction with Heath Consultants (Houston, TX) and the Northeast Gas Association (New York, NY), and with funding from PSE\&G (NJ), SoCal Gas (CA), and the US EPA and DoE, developed an optical methane detector, namely the Remote Methane Leak Detector (RMLD), that provides stand-off detection of leaks with detection capabilities comparable to commonly-used flame ionization detectors. The stand-off range of the RMLD sensor is $\sim 100 \mathrm{ft}$. While several large research programs have investigated the suitability of higher power, usually mid-IR laser-based systems for longer range sensing, this technology has never left the hands of the inventing organization due to the cost and complexity of the associated components. Thus, despite consistent ranking as a highly desired, unmet remote sensing need, the US Natural Gas Industry presently has no access to long range (or high-altitude) remote sensing capability.

In this program, PSI proposed to addresses this need directly with a compact, turn-key, sensor for aerial survey, with possible full autonomy if employed in an unmanned aerial vehicle (UAV). While certainly more expensive than daily leak survey tools, the high-power stand-off sensor will allow regional or national consortia of gas distribution companies to jointly-own or lease the sensor when large area surveys are required. Such capability is expected to vastly improve the efficiency of high capacity distribution line surveys in remote areas and to provide new capability for emergency response in the event of a natural disaster or terrorist activity.

\subsection{Technical Approach}

\subsubsection{Sensor Concept}

The Remote Methane Leak Detector (RMLD), shown in use by Figure 1, is based on the established spectroscopic measurement technology known as Tunable Diode Laser Absorption Spectroscopy (TDLAS). The RMLD includes a handheld optical transceiver and a shouldermounted controller. The transceiver transmits an eyesafe laser beam onto topographic targets up to $100 \mathrm{ft}$. distant, and receives some of the laser light reflected by the target. The controller processes the received light signal to deduce the amount of methane in the laser's path. The entire system weighs a total of approximately $6 \mathrm{lbs}$. Self-contained rechargeable batteries power the device for more than 8 hours continuously on one charge. Field tests of advanced prototype RMLD units commenced with several gas distribution companies in March 2003, with excellent results, and production units have been available from Heath Consultants since Dec 2004. 

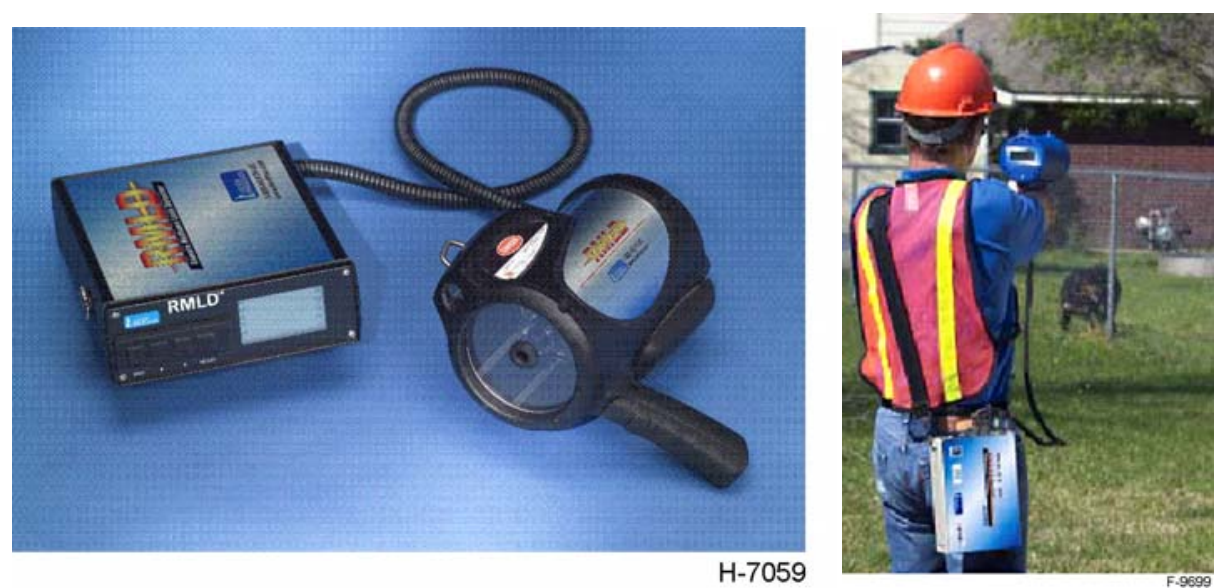

Figure 1. Photographs of PSI's remote methane leak detector (RMLD) production version (left) and advanced prototype during field testing (right).

Physical Sciences Inc. (PSI) proposed to utilize and extend the technology embedded within the RMLD to build and demonstrate a system for standoff sensing, from high altitudes, of natural gas distribution/transmission pipeline leaks. The solid-state, near-IR laser within the RMLD was to be enhanced with a scalable, high-power optical fiber amplifier to provide a compact, power-efficient sensor to be flown upon an aerial platform. In the proposed scope, PSI planned to assemble and flight test a prototype sensor on a UAV having an operational ceiling of $10,000 \mathrm{ft}$. It was later agreed between DoE and PSI that the proposed alternative manned flight test would be a more logical and feasible Phase I testing ground for the prototype. This demonstration was intended to prove the concept and lay the foundation for scaling the device to achieve leak detection from altitudes in excess of 50,000 ft.

Specifically, the sensor was to entail using an existing RMLD electronics board (comprising laser drive electronics and signal processing electronics) with a tunable diode laser at 1.618 micron, coupled to a Er-Doped Fiber Amplifier (EDFA) using single-mode fibers. A 5W EDFA unit was commercially available in a standard 3 in. height, 19 in. rack-mount configuration and weighs approximately $30 \mathrm{lbs}$. A lens assembly was to collect the output from the EDFA and project towards earth a laser beam having a divergence of $6 \mathrm{mrad}$, which, from 10,000 ft will illuminate spot on the ground of $10 \mathrm{~m}$ diameter (or $50 \mathrm{~m}$ from $50,000 \mathrm{ft}$ ). We note that the laser intensity at the ground for this design is approximately $6 \mu \mathrm{W} / \mathrm{cm}^{2}$ - far below eye safety concerns. A receiver telescope, with a 10 in. (or equivalent) diameter, images the illuminated spot onto the near-IR photodetector. The current RMLD circuitry and software will receive the detector signal and process it. Much like the RMLD's two-component design, the laser, EDFA, EDFA power supply, and electronics are all enclosed in a common mechanical housing along with a small portable computer for data recording, and the rest of the components comprise the system transceiver, connected via electrical and fiber cables. Figure 2 illustrates the originally proposed sensor schematic. The most significant technical aspect of the technical approach to the problem is the addition of the high-power EDFA and a majority of the program's early efforts were focused on characterizing the performance of and understanding the limitations of this device when coupled to the overall system. 


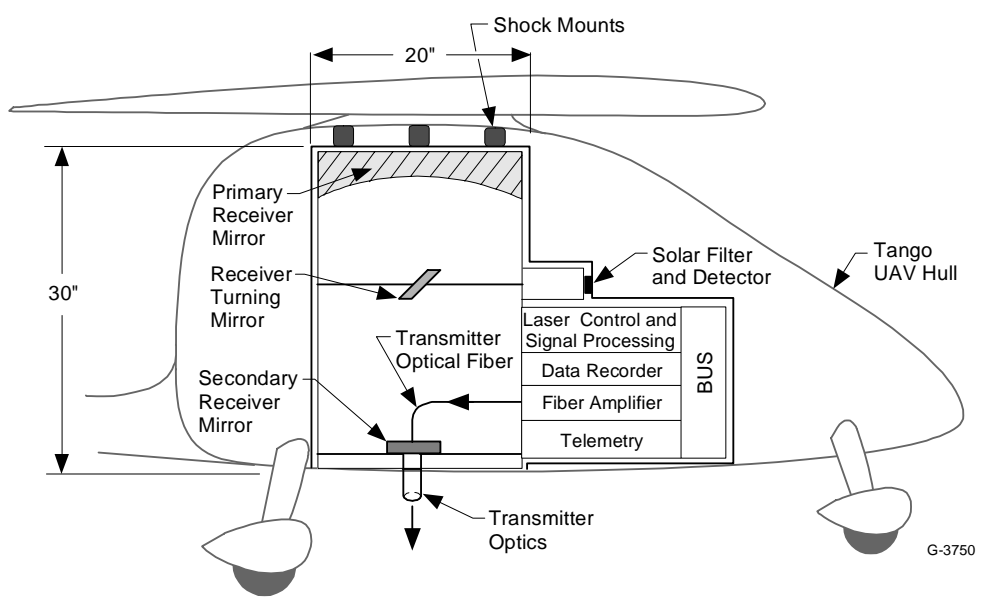

Figure 2. Schematic of airborne amplified RMLD sensor concept.

\subsubsection{Measurement Details}

The RMLD combines a telecommunications-style diode laser, fiber-optic components, and low-cost DSP electronics with the well-understood principles of Wavelength Modulation Spectroscopy (WMS), to indicate, with a sensitivity of 5 ppm-m and $10 \mathrm{~Hz}$ response, the presence of natural gas located between the operator and a topographic target (such as pavement, grass, building walls, etc. The handheld RMLD was designed for a maximum range to the topographic target of $100 \mathrm{ft}$ to accommodate several operational requirements, including weight, size, and power consumption. With this design, laser power collected from targets beyond $100 \mathrm{ft}$ $(30 \mathrm{~m})$ is insufficient to provide a signal-to-noise ratio useful for detecting low-grade leaks. Furthermore, because the RMLD is designed to be comparable in sensitivity to FID, it is also sensitive to the natural methane in the ambient atmosphere, which is typically present at concentrations of about $2 \mathrm{ppm}$. Since the RMLD measures path-integrated concentration, the ambient methane can contribute up to 60 ppm-m (2 ppm x $30 \mathrm{~m})$ of signal, which is comparable to the signal due to a small leak. Operating over longer ranges could thus lead to an inability to discriminate small leaks from ambient methane.

The airborne RMLD is designed to overcome these limitations via an amplification of the laser output power and a reduction of the sensitivity to methane. The prototype version was designed to operate at altitudes up to $10,000 \mathrm{ft}(3 \mathrm{~km})$, where ambient methane would produce a nearly-constant background signal of about 6000 ppm-m. The system design anticipated a signal-to-noise ratio of unity corresponding to about $100 \mathrm{ppm}-\mathrm{m}$, the so-called minimum detection limit. Thus, the prototype version was designed to sense leak plumes having minimum path-integrated methane concentrations of $1000 \mathrm{ppm}-\mathrm{m}$. Over flat-terrain, a variation of this magnitude likely indicates a leak. Thus, this detection scenario was deemed sufficient to detect the presence of a leak from a high capacity transmission line. 


\subsubsection{Technical Foundation}

\section{Tunable Diode Laser Absorption Spectroscopy}

TDLAS instruments rely on well-known spectroscopic principles and sensitive detection techniques coupled with advanced diode lasers, and often with optical fibers. ${ }^{1-4}$ The principles are straightforward: Gas molecules absorb energy at specific wavelengths in the electromagnetic spectrum. At wavelengths slightly different than these absorption lines, there is essentially no absorption. Specifically, when the laser frequency (reciprocal wavelength) is tuned to correspond to a particular absorption transition of the target gas molecule, the transmitted light is attenuated according to the Beer-Lambert relation:

$$
I_{v}=I_{v, 0} \exp \left[-S(T) g\left(v-v_{0}\right) N \ell\right]
$$

where $I_{v}$ is the transmitted intensity at frequency $v$ after propagating through a gas path $\ell, I_{v, 0}$ is the initial laser intensity, $\mathrm{S}(\mathrm{T})$ is the temperature-dependent absorption line-strength (a fundamental spectroscopic property of the molecule), $\mathrm{N}$ is the target species number density, and $\mathrm{g}\left(v-v_{0}\right)$ is the absorption lineshape (describing the spread in frequency of the transition strength). The argument of the exponential function is the fractional change in the laser intensity across the measurement path and is conventionally known as the absorbance. By (1) transmitting a beam of light through a gas mixture sample containing a quantity of the target gas, (2) tuning the beam's wavelength to one of the target gas's absorption lines, and (3) accurately measuring the absorption of that beam, one can deduce the concentration of target gas molecules integrated over the beam's path length. This measurement is often expressed in units of ppm-m.

Practical and robust commercial TDLAS instrumentation came into existence during the 1990 's, made possible by the advent of reliable monochromatic near-infrared (NIR, 1.2 to $2.5 \mu \mathrm{m}$, or 4000 to $8500 \mathrm{~cm}^{-1}$ ) diode lasers that operate continuously and unattended near room temperature. These lasers (specifically the distributed feedback, or DFB, variety that include a grating-like optical element which forces each laser to emit light at a specified NIR wavelength) offer linewidths less than $0.003 \mathrm{~cm}^{-1}$, which is considerably narrower than molecular absorption linewidths that are typically $0.1 \mathrm{~cm}^{-1}$ at atmospheric pressure. Furthermore, by accurately controlling the laser temperature and the electrical current that powers the laser (the "injection current"), the laser wavelength may be tuned rapidly and precisely over a range of about $\pm 2 \mathrm{~nm}$ around its specified wavelength. Typically, each TDL system is built using a laser having a specific design wavelength chosen to optimize the sensitivity to a particular target gas. The wavelength is selected to correspond to a specific absorption line of the target analyte gas that is free of interfering absorption from other molecules.

\section{$\underline{\text { Wavelength Selection }}$}

In the RMLD, the wavelength is near $1.654 \mu \mathrm{m}$, one of methane's strongest near-IR absorption lines. For the airborne sensor, the laser wavelength is changed to enable optical penetration through ambient methane while retaining sensitivity to pipeline leaks. From $50,000 \mathrm{ft}$, the stable atmosphere contributes a methane signal proportional to a column density of $30,000 \mathrm{ppm}-\mathrm{m}$. At the standard $1.654 \mu \mathrm{m}$ wavelength, the ambient methane results in nearly 
$30 \%$ absorption of the laser power - nearly 3000 times larger than the detection limit of the RMLD. Such large absorption will obscure the signal resulting from a gas leak. However, by changing the wavelength to $1.618 \mu \mathrm{m}$, we access a methane absorption line that is $1 \%$ the strength of the $1.654 \mu \mathrm{m}$ line, resulting in an atmospheric background of $0.3 \%$ optical absorption and only about 30 times the sensor's detection limit. This reduction of total atmospheric attenuation is an important feature of the approach in that it reduces the effect of variations in the path-integrated atmospheric background (associated with varying surface topology and altitude) to a smaller optical signal. At this wavelength, the noise-floor of the sensor corresponds to a path-integrated detection limit of $1000 \mathrm{ppm}-\mathrm{m}$. Thus, the sensor will be able to identify leaks of 0.1\% methane in a $1 \mathbf{m}$ deep plume. This is 40 times lower than the explosive limit threshold.

Shifting the detection wavelength from 1.653 to 1.617 microns has several other significant advantages. The chosen wavelength is free from interfering absorption by any standard atmospheric gases, as well as trace gases associated with petrochemical refineries (other than methane, of course), transportation systems, utility power plants, and other industrial operations. Also, additional ambient sources of methane such as livestock, sewer gas, and crop production are typically well below the 1000 ppm-m detection limit and will not be confused with gas leaks.

\section{Wavelength Modulation Spectroscopy}

Fast, sensitive detection of methane is accomplished using the technique of Wavelength Modulation Spectroscopy (WMS), wherein the laser's fast tuning capability is exploited to rapidly and repeatedly scan the wavelength across the selected gas absorption line. While this periodic wavelength modulation occurs, the fraction of emitted laser power that is transmitted through the atmosphere is monitored with a photodetector. When the wavelength is tuned to be off of the methane absorption line, the transmitted power is higher than when it is on the line. Because each cycle of the modulation causes the wavelength to cross the absorption feature twice, the resulting amplitude modulated signal is periodic with a fundamental frequency of twice the wavelength modulation frequency. In the literature, the fundamental component is called the $2 \mathrm{f}$ signal. Phase sensitive (lock-in) detection accurately measures the amplitude of the $2 \mathrm{f}$ signal, which depends on both the power of the transmitted beam and the path-integrated concentration of methane. $2 \mathrm{f}$ signals representing absorption of $1 / 100,000$ of the average received laser power are detected routinely by this technique. The average value of the received laser power, $\mathrm{P}_{\mathrm{DC}}$, is measured separately and utilized to normalize the $2 \mathrm{f}$ signal. The resulting ratio depends only on path-integrated methane concentration.

\section{Remote Sensing}

All of the circuitry and processing hardware required to operate the laser and implement WMS is, in the RMLD, contained on a single 6 inch square printed circuit board. All of the laser control, thermal control, signal processing, and data reporting functions are performed on this board, which draws only $1.5 \mathrm{~W}$ of power. The laser source is mounted on the board. The laser output light is transmitted via optical fiber to a separate optical transceiver (see Figure 1), which projects the laser beam and receives the reflected light. The transmitted laser power is limited to $10 \mathrm{~mW}$ for eye-safety considerations. 
To adapt the RMLD for high altitude use in the airborne platform, three aspects are modified: 1) The transmitted laser power is increased by use of an optical fiber amplifier; 2) The size of the optical receiver is increased; and 3) The laser wavelength is changed. Equation (1), which relates the received laser power to the transmitted power, optical receiver size, and operating distance, provides the rationale for the first two changes:

$$
P_{D C}=\left(\frac{A_{c o l} R_{\text {dif }} \eta_{\text {opt }}}{R^{2}}\right) P_{\text {out }}
$$

where:

$$
\begin{aligned}
\mathrm{A}_{\text {col }} & =\text { effective area of optical receiver in } \mathrm{m}^{2} \\
\mathrm{R}_{\text {dif }} & =\text { differential reflectance of topographic targets } \\
\eta_{\text {opt }} & =\text { optical efficiency } \\
\mathrm{R} & =\text { distance to topographic target in } \mathrm{m} \\
\mathrm{P}_{\text {out }} & =\text { laser output power in } \mathrm{W}
\end{aligned}
$$

The RMLD achieves a $5 \mathrm{ppm}-\mathrm{m}$ detection limit at $30 \mathrm{~m}$ using absorption from one of the strongest near-IR transitions of methane, a 4-in. diameter receiver optic, and $\mathrm{P}_{\text {out }}=10 \mathrm{~mW}$. Equation (1) shows that the collected power scales as the inverse of distance squared, so to achieve a comparable detected photocurrent at $50,000 \mathrm{ft}(\sim 15,000 \mathrm{~m})$, we would require $2.5 \mathrm{~kW}$ of laser power with the same 4 -in. receiver, but would need only $125 \mathrm{~W}$ with an 18 -in. diameter receiver. This laser power and mirror diameter is achievable with scalable fiber amplifier technology and a compact sensor payload consistent with anticipated future UAV's. They are, however, beyond the scope of this program, where we limit the laser power to $5 \mathrm{~W}$ and the effective mirror diameter to $10 \mathrm{in}$., sufficient to demonstrate leak detection from an aircraft flying at 10,000 ft. The present prototype can be scaled up in EDFA output power for higher altitudes and specific UAV platforms.

\section{Erbium-Doped Fiber Amplifier}

Finally, and most importantly, the shorter operating wavelength allows us to take advantage of rapid and ongoing progress in telecommunication fiber-amplifier technology to achieve the needed laser output power. Erbium-doped fiber amplifier (EDFA) technology is widely used in the optical telecommunication to boost the operating power of semiconductor lasers operating in the Wavelength Division Multiplexing (WDM) bands from 1.530 to 1.620 microns. Using well-behaved single-mode fibers, high-efficiency and long-life diode pump lasers, commercial EDFA's are available today with output power up to $150 \mathrm{~W} .^{5}$ Recent advances in fiber laser research have produced devices operating in excess of $1 \mathrm{~kW}{ }^{6}$ These compact devices have been specifically engineered for the demanding requirements of long-haul telecommunication applications, including $>10 \%$ wall-plug power efficiency, $>50,000$ hours operating lifetimes, all air-cooled packaging. No other laser technology can provide these specifications. We employ a commercial $5 W$ EDFA in the prototype sensor. 


\subsection{Program Scope}

\subsubsection{Program Objectives}

The principal objective of the proposed program is to develop and demonstrate costeffective and power-efficient advanced remote sensing technology able to detect and quantify, from an airborne platform, natural gas leaking from a high-pressure pipeline. An extension of already-proven diode laser-based standoff detection, the technology demonstrated in this program was originally intended to be scalable to use on UAVs operating at 50,000 ft and higher.

Physical Sciences Inc. (PSI) was to utilize and extend the technology embedded within the handheld, battery-powered laser-based Remote Methane Leak Detector (RMLD) product, developed by PSI, to build and demonstrate a system for such airborne standoff natural gas sensing. The solid-state, near-IR laser within the RMLD was to be enhanced with a scalable, high-power optical fiber amplifier to provide a compact, power-efficient sensor amenable to employment in an aircraft. In the revised proposed program, PSI was to assemble and flight test a prototype sensor on a manned aircraft having an operational ceiling of 10,000 ft. This demonstration was to prove the concept and lay the foundation for scaling the device, in a subsequent phase, to achieve leak detection from altitudes in excess of 50,000 ft.

The specific Program Objectives were to:

1. Define the overall system architecture and specifications.

2. Construct a flightworthy prototype sensor.

3. Perform laboratory and ground-based outdoor sensor characterization tests.

4. Prepare for and perform a flight test of the sensor.

5. Communicate the results to DoE/NETL

The technical structure of the program was divided into the following seven Program Tasks:

1. Create a Research Management Plan.

2. Perform a Technology Status Assessment.

3. Define the overall system architecture and specifications.

4. Design, specify and acquire components for, and assemble a prototype sensor.

5. Perform laboratory and ground-based outdoor sensor characterization tests.

6. Make preparations for sensor installation and flight testing.

7. Perform the flight test, data analysis, and altitude scaling.

The actual program deviated from plan at Task 7. Unfortunately, PSI received notification just prior to the scheduled flight test that the leak-providing pipeline operator was withdrawing from the planned test. PSI was unable to secure an alternative test site where a significant leak could be created, and therefore abandoned the planned flight test. In lieu of this test, PSI performed a ground-based simulation of a low-altitude aerial survey scenario. This simulated flight test is described in the following section and the results of the tests are documented in Section 3. 
As detailed in Section 3 below, most of the program objectives were met. Several technical issues were identified that would require engineering improvements in the laser amplifier to match the predicted sensor performance for high-altitude ( $>1000 \mathrm{ft})$ aerial survey, though the sensor as constructed is predicted to be employable at such altitudes with somewhat lesser sensitivity characteristics. However, the simulated flight test data illustrate that lowaltitude leak survey should have excellent leak survey applicability.

\subsubsection{Program Tasks}

As listed above, the program comprised seven tasks, the sum of which, plus the present and previous reports, are dedicated to the completion of the abovementioned technical objectives. One deviation from the proposed plan was required so the Tasks reported here are as they were performed in the program.

\section{Task 1. Create a Research Management Plan}

This task was intended to establish a plan that guided the program work, schedule, and budget, facilitated communication among team members, and enabled progress to be tracked. PSI was to develop a work breakdown structure (WBS) and supporting narrative that concisely addressed the overall project. The narrative was to summarize the technical objectives and technical approach for each Task and, where appropriate, for each subtask. The plan was to include schedules and expected expenditures for each Task, plus all major milestones and decision points. The report was to be submitted to the DOE Contracting Officer's Technical Representative (COTR) for review and comment, and be subsequently finalized incorporating any DoE input.

\section{Task 2. Perform a Technology Status Assessment}

In this Task, PSI was to review and communicate the state-of-the-art of the technology to be developed in this program to clearly communicate the advancements that the research is intended to achieve. PSI was to review the state-of-the-art of airborne surveying for natural gas pipeline leaks and submit a Technology Status Assessment summary report. The short report was to include both positive and negative aspects of each existing technology. This report was to be submitted to the DOE Contracting Officer's Technical Representative (COR) for review and comment, and be subsequently finalized incorporating any DoE input.

\section{Task 3. System architecture and specifications}

The objective of this Task was to create a written document describing, in the form of a set of specifications, the technology to be developed meeting the program goals. This specification document would guide the design of the technology system, serve as a basis for ongoing discussions about the technology, and could be modified or refined as the technology development progressed. PSI was to identify the major system components and the aircraft to be used for flight tests in Task 7. The major components were to be selected to be compatible with aircraft size, weight, and power requirements. Based on these components, PSI would determine the expected detection range, sensitivity, footprint at ground, and operational speed. This 
information would be documented in a Preliminary Specification. A draft of the specification document was to be submitted to the DOE Contracting Officer's Technical Representative (COR) for review and comment, and subsequently to be modified as the program progressed, incorporating any DoE input.

\section{Task 4. System design, acquisition, and assembly}

The goal of this Task was to design and build the Experimental Prototype (EP) leak sensor that would be flight tested in Task 7. This Task was comprised of the following six Subtasks intended to result in a robust a verified device suitable for field testing:

Benchtop sensor assembly and preliminary laboratory testing - Major components assembled in a benchtop laboratory configuration and tested to verify functionality and collective performance.

Preliminary Airborne Package Design - Preliminary design with top level assembly drawings of the airborne sensor configuration.

Preliminary Design Review - Preliminary design presented to an audience of experts for review and critique, and the design revised as appropriate.

Detailed Design of the Engineering Prototype - Final design with top level assembly drawings and bill of materials (BoM) of the airborne sensor configuration.

Design Review - Final design presented to an audience of experts for review and critique, and the design revised as appropriate.

Assemble Engineering Prototype - Assemble the sensor according to the revised final design.

\section{Task 5. Laboratory and outdoor ground testing}

In this Task, PSI was to test and optimize the performance the EP system built in Task 4 and evaluate the readiness for flight testing. The EP system would be tested over long horizontal paths, with the laser beam safely transmitted to distant topographic targets of convenience. The system was to be calibrated and optimized for evaluation of noise contributions, methane sensitivity, interferences, and collection efficiency. Detection algorithms would be accommodated to overcome rapidly changing background signals. The algorithms (improved over initial RMLD algorithm development) would be provided to this program as part of the PSI cost sharing contribution. Ground test results were to be compared against the system specifications (as defined in Task 3 ) and the system and specifications refined as required. PSI would determine whether the system as built would meet the requirements for airborne testing. The data and conclusions will be presented to the DOE Contracting Officer's Technical Representative (COTR) for review and comment. Upon completion of the review, a decision to proceed with flight testing or not would be taken. 


\section{Task 6. Flight test preparation}

PSI was to prepare the Engineering Prototype system for flight testing. This would include coordinating with the aircraft operator to specify details such as power connections, installation supports, safety considerations and flight test protocols. PSI and the aircraft operator would define a limited set of shock and vibration tests that the sensor system must survive prior to flight to assure flight safety. Upon completion of the safety tests and any re-configuration of the sensor to accommodate aircraft installation, a final ground test just prior to flight would be completed to assure and optimize sensor performance. In this Task, PSI would also prepare test protocols, arrange test sites, and contract with the aircraft provider to conduct two flight test series, each with methane cloud flyovers of at least three different altitudes.

\section{Task 7. Simulated flight test}

In lieu of the cancelled flight test, PSI was to conduct a ground-based simulation of a low-altitude (short-range) flight test. The experiment would employ a methane-filled plastic bag mounted on a automobile that drives through an unamplified sensor laser beam at speeds comparable to a slow-flying aerial survey aircraft. PSI would analyze the data from these experiments to determine sensitivity, altitude scaling and compare the unamplified EP actual performance against design optimums.

To complete the program requirements, aside from the technical Tasks listed above (including the three documents of Tasks 1-3), PSI was also to deliver Financial Status Reports on a quarterly basis, Progress Reports on a semi-annual basis, and a Final Technical Report (this document). 


\section{PROGRAM RESULTS}

\subsection{Program Results Summary}

As further detailed in the submitted semi-annual reports and below in Section 3.2, this program covered the following list of efforts and experiments:

- Research management plan

- Technology status assessment

- Spectral analysis, wavelength selection, and sensitivity estimate

- Amplifier selection and characteristics

- Aircraft selection

- Optical components selection

- Transceiver components configuration

- System specification

- DMD algorithm development

- Seed laser characterization

- Amplifier characterization

- Assembly and alignment of the high-altitude optical transceiver

- Combination of the transceiver and amplified laser to form the complete sensor system

- Performance of outdoor ground field tests

- Measurement of signals from long range $(2000 \mathrm{~m})$

- Detection of transient methane leaks

- Prototye system performance characterization

- Exploration of amplifier operating parameter space for noise and drift reduction

- Completion of flight test preparations

- Creation of surrogate flight test and simulated flight test plans for cancelled flight test

- Performance of simulated (ground-based moving target) flight tests with unamplified laser

- Data analysis and sensitivity estimate calculations

\subsection{Detailed Results}

The details of the results of the above efforts are broken out in this Section by program Task.

\subsubsection{Task 1: Research Management Plan}

The Research Management Plan was the first Task completed and served as a guide for program organization and progress. This document is attached to this report as Appendix A. It includes the program Task list, proposed timeline, cost and spending breakdowns, and lists of milestones and deliverables. 


\subsubsection{Task 2: Technology Status Assessment}

The Technology Status Assessment is attached to this report as Appendix B. Its purpose was to evaluate the state-of-the-art in laser-based and aerial natural gas leak detection, and to understand how the enhanced RMLD technology to be developed in this program would provide a significant advancement to the field.

\subsubsection{Task 3: System Architecture and Specifications}

An initial Sensor Specification document was created after some of the earlier elements of Task 4, which included the selection of various components of the optical system. This document was further revised a few times during the early design stage and the most recent version is attached to this report as Appendix C. It represents the expected generic design and performance of the ideal system, as modeled, and does not incorporate any of the real-world performance limitations observed experimentally with the hardware employed in this program.

\subsubsection{Task 4: System Design, Acquisition, and Assembly}

\section{Spectral Analysis, Wavelength Selection, and Sensitivity Estimate}

This project requires EDFA technology, which is limited to wavelengths below $1625 \mathrm{~nm}$, and access to methane absorption lines that are free from atmospheric interference. Figure 3 shows the methane absorbance in the $1614-1625 \mathrm{~nm}$ spectral region, computed for $1 \mathrm{ppm}-\mathrm{m} \mathrm{CH} \mathrm{CH}_{4}$ at $300 \mathrm{~K}$ using the HITRAN 96 database and TRANSCD plotting package. These methane features are approximately two orders of magnitude weaker than the standard RMLD absorption line at $1654 \mathrm{~nm}$. A well-isolated line is located at $1618.0 \mathrm{~nm}$. As illustrated in Figure 4, this line is also distinct from nearby water and carbon dioxide lines. The $1618 \mathrm{~nm}$ line is, therefore, the preferred line for the airborne RMLD.

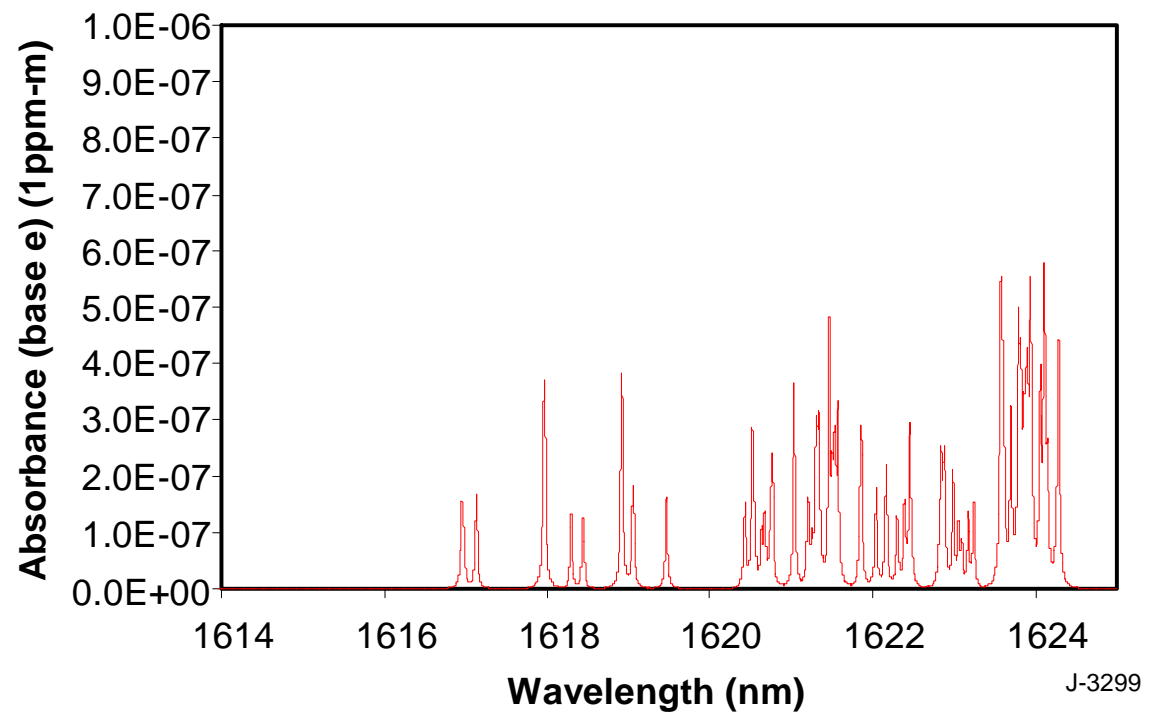

Figure 3. Methane absorption for 1 ppm-m at $300 \mathrm{~K}$. 


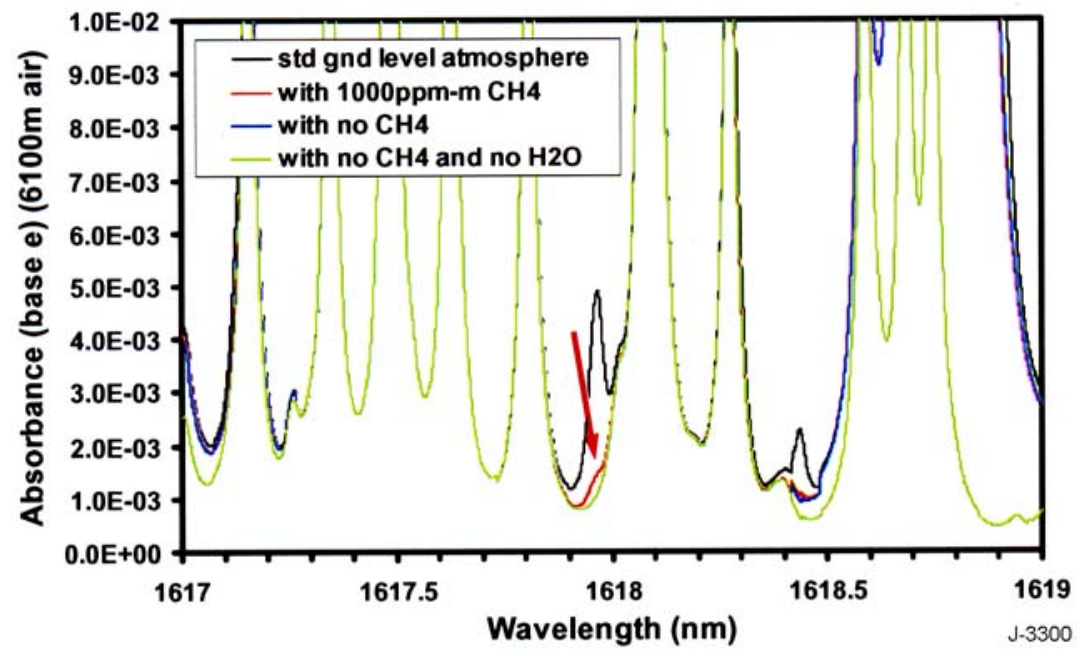

Figure 4. Detailed examination of atmospheric absorption at $300 \mathrm{~K}, 6100 \mathrm{~m}$ pathlength. The target methane feature is on the shoulder of the stronger $\mathrm{CO}_{2}$ transition near $1618 \mathrm{~nm}$.

Figure 4 plots the atmospheric absorbance at a pathlength of 10,000 ft (6100 m optical round trip), for the spectral region around $1618 \mathrm{~nm}$ for (a) standard ambient air concentrations, including $1.7 \mathrm{ppm} \mathrm{CH}_{4}$ (10370ppm-m), (b) a "sample leak" $1000 \mathrm{ppm}-\mathrm{m} \mathrm{CH}_{4}$ contribution (no background $\mathrm{CH}_{4}$ ), (c) with no $\mathrm{CH}_{4}$ contribution, and (d) with no $\mathrm{CH}_{4}$ and no $\mathrm{H}_{2} \mathrm{O}$ contribution (to illustrate the location of the water lines). The RMLD platform has a minimum detectable absorbance of about $10^{-4}$. At $1618 \mathrm{~nm}$, a path-integrated methane concentration of 250 ppm-m provides an equivalent $10^{-4}$ absorbance. The $1000 \mathrm{ppm}-\mathrm{m}$ "leak" yields a signal that is 4 times the RMLD noise floor $\left(4 \times 10^{-4}\right.$ absorbance) at $1618 \mathrm{~nm}$ and is barely visible on the shoulder of the $\mathrm{CO}_{2}$ line in Figure 4. As illustrated, the atmospheric $\mathrm{CO}_{2}$ absorbance is $\sim 1.0 \mathrm{x}$ $10^{-3}$ at $1618 \mathrm{~nm}$ for this pathlength and is representative of a 10,000ft altitude survey, but the $\mathrm{CH}_{4}$ concentration decreases with altitude, so the atmospheric methane absorbance will actually be about $1.2 \times 10^{-3}$ (significantly lower than the $3.8 \times 10^{-3}$ illustrated (black curve)). This background $\mathrm{CH}_{4}$ concentration is 12 times the noise floor of the RMLD unit, corresponding to about $3000 \mathrm{ppm}-\mathrm{m}$. For an aircraft flying at this fixed altitude, this atmospheric absorbance is a constant signal upon which variations due to leaks or changes in terrain are superimposed. Assuming terrain changes by less than $10 \%$ of the flight altitude (i.e. altitude above ground is constant to within $10 \%$ ), then increases in methane concentration of nominally $20 \%$ above the atmospheric background are attributable to leaks. Thus, we expect with the airborne RMLD to detect leaks that produce plumes having path-integrated concentrations larger than about 600 ppm-m. Since the laser beam passes through a gas cloud twice on its path from and back to the transceiver, this can be thought of as a single-pass path-integrated concentration of 300 ppm-m (with a 125 ppm-m anticipated noise limit).

This $10^{-4}$ limiting absorbance is typically observed for the $10 \mathrm{~Hz}$ data rate and detector noise limiting condition (low return signal). The calculated noise-limited concentrations above are also in good agreement with modeling of the signals and noise sources in the WMS process, as shown in Figure 5, where the signal/noise ratio (SNR) for a $1000 \mathrm{ppm}-\mathrm{m} \mathrm{CH}_{4}$ plume is in the 2 to 4 range for the detector noise-limited signal range. 


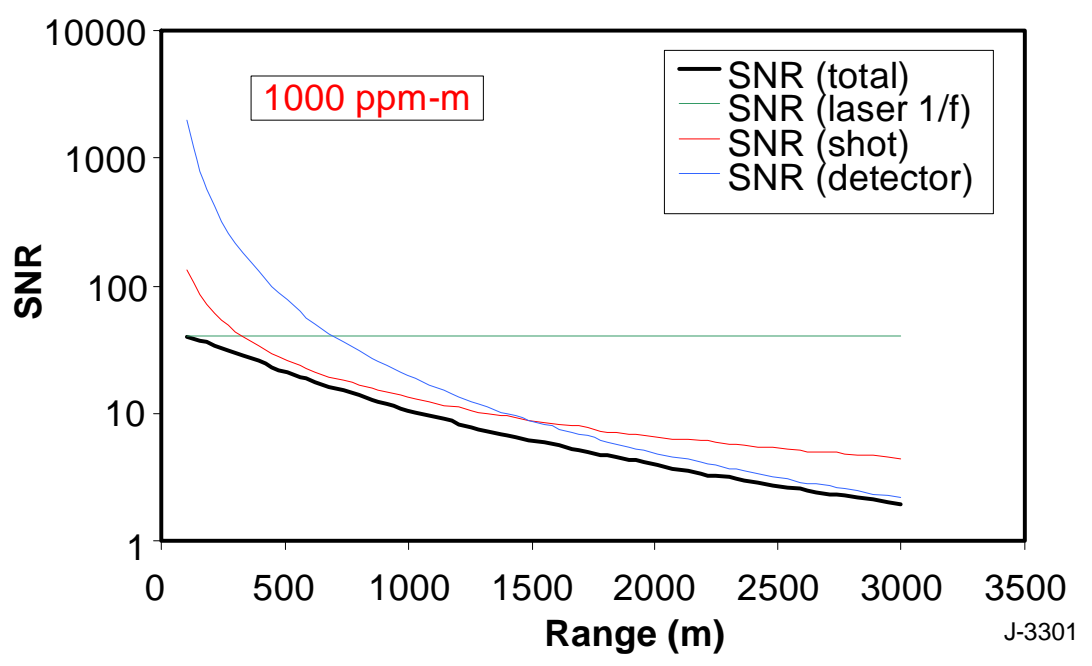

Figure 5. Modeled signal/noise ratio for a 1000ppm- $\mathrm{m} \mathrm{CH}_{4}$ plume versus sensor range-totarget. In this model, a differential reflectance of $0.2 \mathrm{sr}^{-1}$ was employed, as well as a $40 \mathrm{~nm}$ bandwidth sunlight blocking filter and a $1.5 \mathrm{~mm}$ InGaAs detector.

In summary, in this Task we compared the spectral absorbance from methane with absorbance by other atmospheric molecules and settled on a wavelength for detecting methane that minimizes cross-sensitivities. With this wavelength selection, we were able to estimate the sensitivity to methane leaks (as described above), and to specify and order the required laser amplifier (described below).

\section{$\underline{\text { Laser Amplifier Selection and Characteristics }}$}

The wavelength of our laser, $1618 \mathrm{~nm}$, is somewhat beyond the standard telecommunications wavelengths range where commercial EDFAs are readily available.

Obtaining at 5W EDFA at that wavelength was a small extension of the state-of-the-art. We obtained quotes for the device from two vendors, Keopsys Inc. (Clinton, NJ) and IPG Corp. (Sturbridge, MA). Based on their previous experience working at that wavelength, performance specifications, cost, and delivery schedule, we chose Keopsys. The quoted specifications of the EDFA that we ordered are:

- Operating Wavelength: 1616-1618nm

- Input Power: $\geq 10 \mathrm{~mW}$

- $\quad$ Input Linewidth: $>1 \mathrm{MHz}$

- Output Power: 5W CW (signal), over operating wavelength range.

- Output Bandwidth: $<1 \mathrm{GHz}$

- $\quad$ Beam Quality: $\mathrm{M}^{2}<1.1$

- Power Stability: $<5 \%$ peak to peak over 4 hours at fixed temperature and output power

- Input isolation (>20dB)

- Output isolator

- Fiber Type: Standard single mode

- Input / Output Fiber Length: 1m 
- Input Connectors FC/APC

- Output Connectors FC/APC with expanded mode fiber termination

- Supply Voltage: $28 \mathrm{~V}$ DC

- Power Consumption: $<130 \mathrm{~W}$

- Operating Temperature: $5^{\circ} \mathrm{C} /+35^{\circ} \mathrm{C}$

- Storage Temperature: $-20^{\circ} \mathrm{C} /+70^{\circ} \mathrm{C}$

- Size: 446x448x88mm (19' rack-mount)

The unit was delivered during the first week of May 2005. Figure 5, acquired by Keopsys prior to shipment, shows the spectral output of the EDFA when seeded with $10 \mathrm{~mW}$ of input power at $1617.8 \mathrm{~nm}$ supplied by an External Cavity Diode Laser (ECDL). The output power plotted on the ordinate of Figure 6 is attenuated; the actual peak power is $5.1 \mathrm{~W}(37 \mathrm{dBm})$.

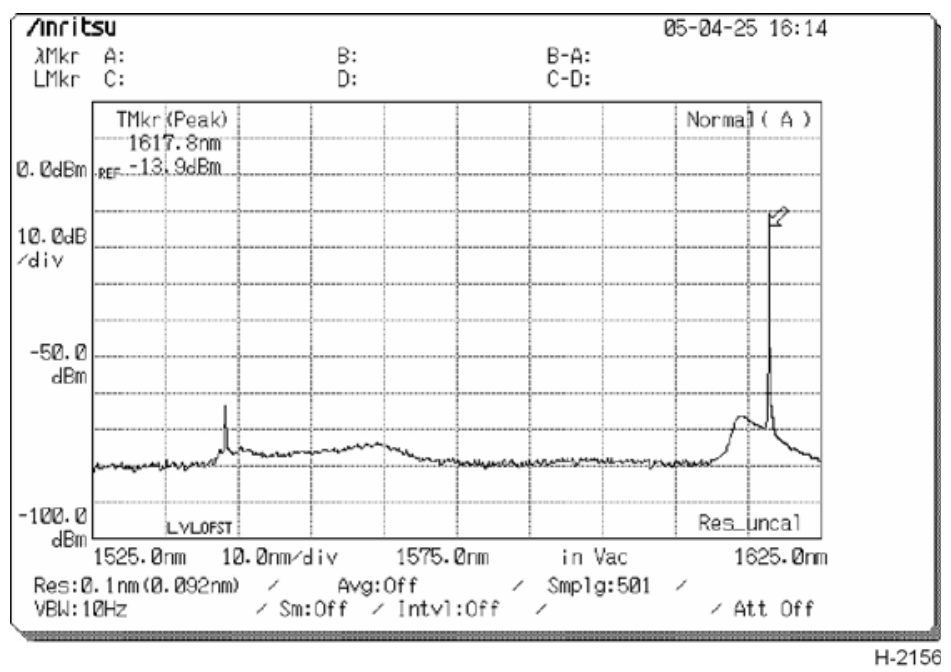

Figure 6. EDFA Output Spectrum

\section{$\underline{\text { Aircraft Selection }}$}

In the Aircraft Selection task, we searched for aircraft suitable for testing the airborne leak detection system and identified two aircraft for potential use in testing the airborne RMLD. One was a Cessna O-2 operated by Aurora Air Services of Manassas, VA and the other was a Cessna 207 operated by Gamm Air of Pottstown, PA. Though both have floor penetrations for accommodating aerial survey equipment and electrical power, $\mathrm{AC}$ and $\mathrm{DC}$, we settled on the Gamm Air platform (see Figure 7) as ideal. Its factory-installed penetration is 22 inches in diameter, large enough to accommodate our initial transceiver design that incorporates a 10" diameter receiver and components to be mounted around the receiver. In contrast, the Aurora aircraft has a rectangular penetration smaller than our receiver. The aircraft would have required additional modification to fully meet our requirements. 

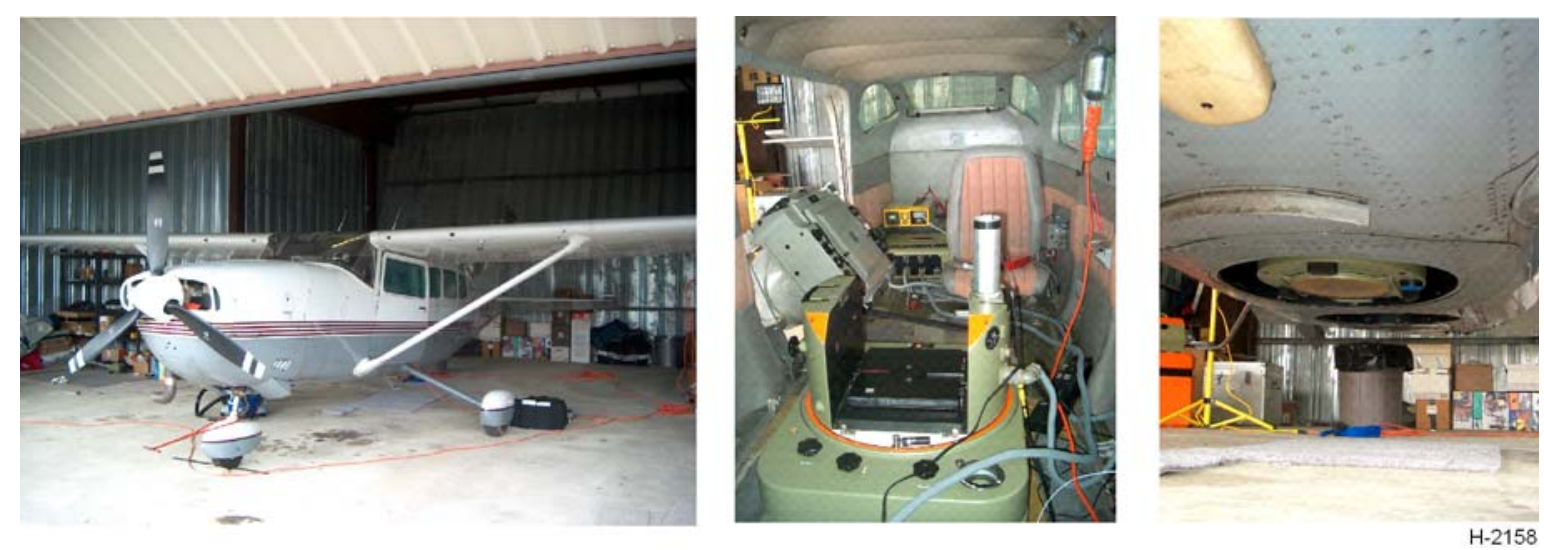

Figure 7. Gamm Air's Cessna 207, cabin with photographic equipment installed, and exterior view of camera port

With knowledge of the aircraft dimensions and utilities, we could specify and purchase the components for the optical transceiver and design a configuration for straightforward installation in the aircraft.

\section{Optical Components Selection}

Based on the availability of the Cessna 207 and its capability to accommodate a 10 inch diameter 48 inch long optical receiver, we specified and purchased optical components to complete the configuration described in the Sensor Specification attached as Appendix C. The optical receiver is a Celestron C10-N Newtonian telescope, featuring a 10" diameter, $1200 \mathrm{~mm}$ focal length $(\mathrm{F} / 4.7)$ parabolic primary mirror. In use, the telescope eyepiece is replaced with the RMLD photodetector. The selected camera is an Edmund Scientific Model NT39-244 black and white CCD camera $(36 \times 36 \times 63 \mathrm{~mm})$ with $60 \mathrm{~Hz}$ video output. These two components are shown in Figure 8. The selected camera lens was an Edmund Scientific Model Y56-531 lens having a $50 \mathrm{~mm}$ focal length and $7.3^{\circ}$ field of view. This permitted a field of view significantly $(\sim 36 \mathrm{x})$ wider than the designed laser beam footprint, whose initial designed divergence was $\sim 0.2^{\circ}$ (3.33mrad). A BNC cable connects the analog camera output signal to a $1^{\text {st }}$ Vision Inc. Model VCE-B5A01 frame grabber. This is a PCMCIA card that plugs into a laptop computer. C-callable subroutines were supplied with the frame grabber.

The optical transmitter (laser launch) was designed around a single component collimation optic, an off-axis parabolic mirror (Janos A8037) that collects and collimates the output laser beam from the EDFA output fiber, turning the beam $90^{\circ}$ in the process. The limiting collimatibility (or beam divergence) of the launch is dictated by the output fiber diameter $(\mathrm{a}=$ $40 \mu \mathrm{m})$ ) and the focal length of the collimating lens via the relation $\theta_{\text {div }}=\mathrm{a} / \mathrm{f}$, dictating a focal length of at least $12 \mathrm{~mm}$ was required. The selected mirror has a 2 " focal length and 1" diameter. The $\sim 0.22 \mathrm{NA}$ of the output fiber yields a beam diameter at the optic of $2 \mathrm{x} \mathrm{x} \mathrm{NA}=22 \mathrm{~mm}$. 

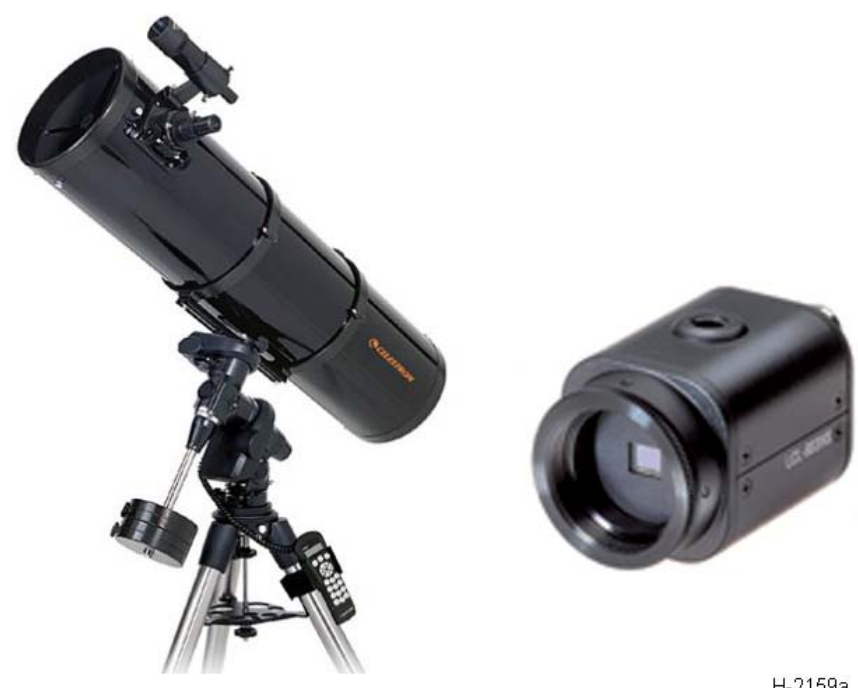

H-2159a

Figure 8. Celestron C10-N telescope (left) and Edmund Scientific Model NT39-244 black and white CCD camera (right).

With a magnification $(\mathrm{m})$ of 2500 at the $3000 \mathrm{~m}$ range $(\mathrm{m}=3000 \mathrm{~m} / 1.2 \mathrm{~m}$ f. 1.$)$, the image of the $10 \mathrm{~m}$ laser spot at the detector is $\sim 4 \mathrm{~mm}$ in diameter. Initially, a $5 \mathrm{~mm}$ InGaAs detector was considered for simplicity's sake, but for better noise performance, a secondary focusing lens (6.24mm f.1., 0.4 NA) was employed near the image plane to focus onto the familiar (RMLD) $1.5 \mathrm{~mm}$ detector. This is illustrated in the optical raytrace modeling (Zemax) shown in Figure 9. Also, just in front of the lens position, we employed a 1" diameter bandpass filter (Spectrogon) with a center wavelength of $1615 \mathrm{~nm}$ and bandpass (FWHM) of $40 \mathrm{~nm}$ for reduction of sunlight at the detector.

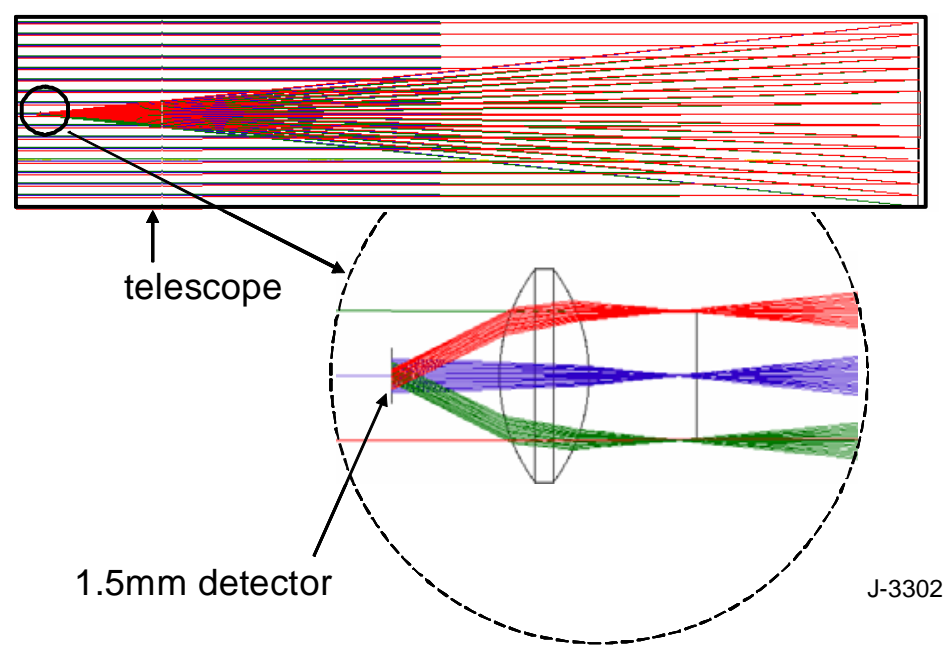

Figure 9. Raytrace analysis of rays from $10 \mathrm{~m}$ object at $3000 \mathrm{~m}$ range. Blue rays are from the object center. Red and green rays are from points at the edge of the object (5m offcenter). 


\section{Optomechanical Design}

We designed a simple mounting apparatus for the laser launch that mounts on the side of the receiver telescope body, illustrated by Figure 10, to attach and align the transmitter components (and thereby the laser beam) with the telescope field of view. The optical fiber termination attaches to a standard fiber bulkhead mount and transmits the beam into a cylindrical channel that ends at the off-axis parabolic mirror. The bulkhead screws into a threaded section of the cylinder, enabling precise positioning of the fiber with respect to the mirror, thereby enabling precise control over the divergence of the laser beam reflected from the mirror. The transmitter assembly mounts to a pair of plates with screw adjustments enabling horizontal and azimuthal alignment of the transmitter optical axis with the receiver optical axis. With a required pointing accuracy of $1 \mathrm{~m}$ at the $3000 \mathrm{~m}$ altitude $(300 \mu \mathrm{rad})$, fine pitch adjustors of $600 \mu \mathrm{rad} / \mathrm{turn}$ were incorporated.
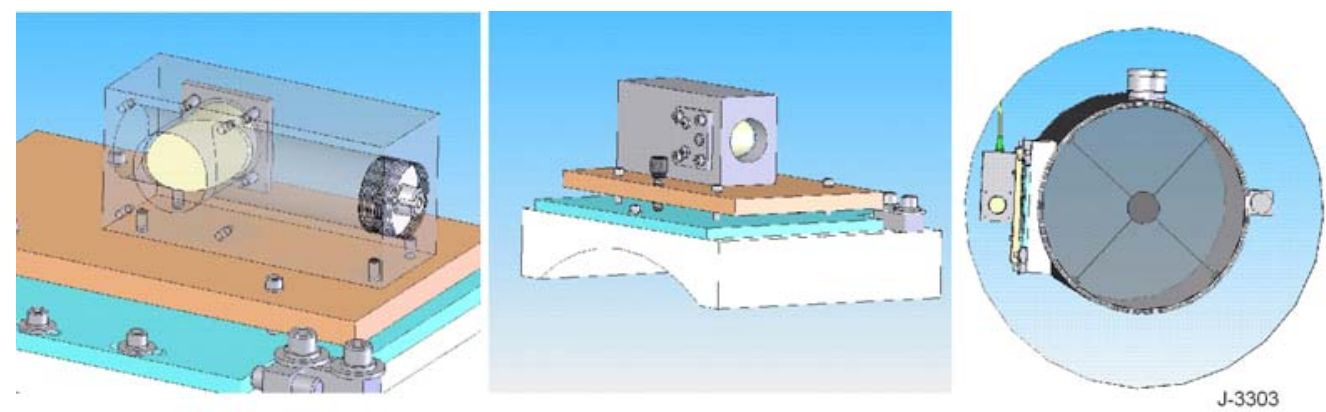

Figure 10. Laser beam transmitter apparatus.

With the beam launch thus positioned $\sim 8$ " off the center of the telescope, some lack of overlap between transmitted beam and received field of view was anticipated for shorter ranges. With the beam centered on the optical axis of the telescope at $3000 \mathrm{~m}$, the anticipated collection efficiencies for different ranges are as illustrated in the raytrace results of Figure 11. In practice, including the short-range flight test simulation detailed in Section 3.7, the short range measurements had the laser centered in the telescope field of view at $\sim 200 \mathrm{~m}$ and the detector backed out as far as possible, bringing the collection efficiency back to the $\sim 100 \%$ level.

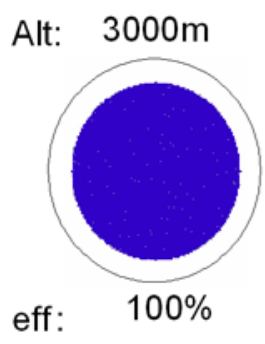

focus: $1200 \mathrm{~mm}$

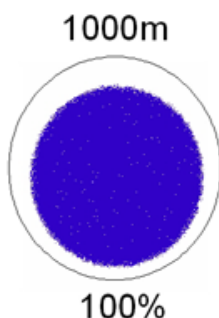

$1201 \mathrm{~mm}$

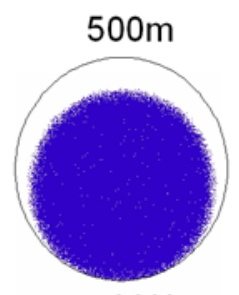

$99 \%$

$1203 \mathrm{~mm}$

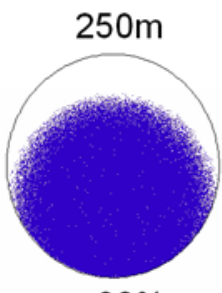

$90 \%$

$1206 \mathrm{~mm}$

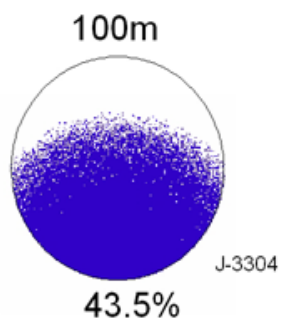

$1215 \mathrm{~mm}$

Figure 11. Raytrace images at the detector plane (and related collection efficiencies and focal plane locations) for various sensor-to-target ranges. 
Figure 12 illustrates the overall sensor configuration for installing the system in the Gamm Air Cessna 207. The EDFA and other electronics will mount in a rack securely located directly next to the optical transceiver. The $1 \mathrm{~m}$ length of the EDFA output fiber limits the separation between the two system components.

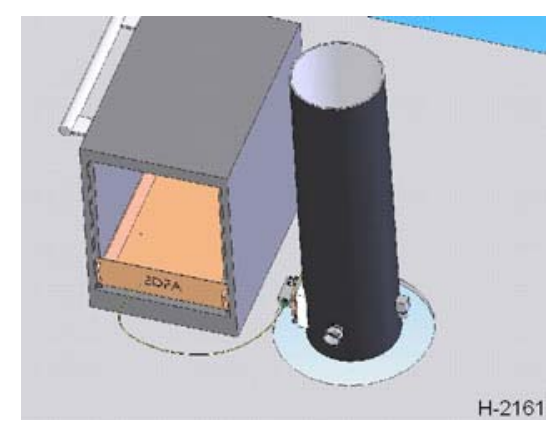

Figure 12. Airborne RMLD system mounting configuration

\subsubsection{Task 5: Laboratory and Outdoor Testing}

\section{Seed Laser Characterization}

PSI has contributed to this program the seed laser and RMLD control platform that provides the input signal to the EDFA and analyzes the output of the receiver. In this Subtask, we completed building and testing this system. The seed laser provides $22 \mathrm{~mW}$ of laser power at the $1618 \mathrm{~nm}$ wavelength, more than double the $10 \mathrm{~mW}$ needed for input to the EDFA. For characterization purposes, we coupled this unamplified platform to a benchtop version of an RMLD transceiver as well as an optical cell. A schematic of most of the components utilized for the laboratory benchtop experiments is shown in Figure 13.

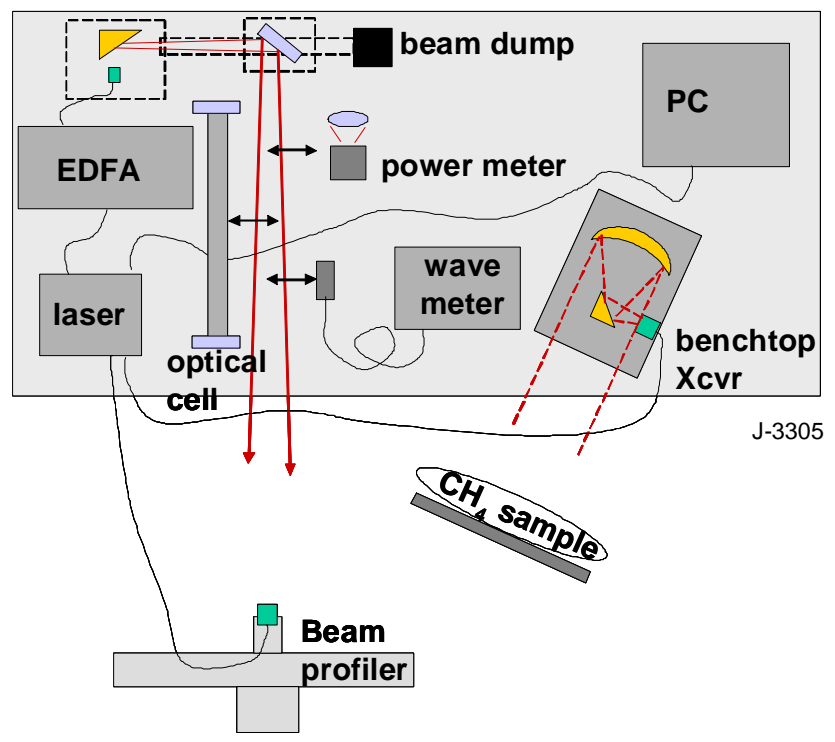

Figure 13. Schematic of laboratory benchtop hardware for airborne RMLD component characterization. 
Initial coupling of the unamplified system to the benchtop transceiver verified the ability to sense methane. We tuned the laser transmitter system to modulate across the selected methane line with laser operating parameters of average current $=120 \mathrm{~mA}$, modulation current $=14 \mathrm{~mA}$, and temperature $=34.4 \mathrm{C}$. We aimed the transceiver at a gray door about $5 \mathrm{~m}$ from the transceiver and launched the full $22 \mathrm{~mW}$ of laser power. The transceiver received about $50 \mathrm{nW}$ of return power. We filled a transparent Tedlar bag to a thickness of about $10 \mathrm{~cm}$ with $100 \%$ methane. Thus, when the laser beam passes back and forth through the thickness of the bag, it transits about 200,000 ppm-m of methane. Figure 14 illustrates the path-integrated concentration output by the system. The red data show the data with the bag inserted in the optical path. We used these data as a very coarse initial calibration - in other words, we set the system calibration constant so the average value of these data is $200,000 \mathrm{ppm}$. We then removed the methane bag from the optical path and measured the system noise in units of ppm-m, calculated using the coarse calibration. The record of this data is shown in green. They indicate an rms noise level of $490 \mathrm{ppm}-\mathrm{m}$, comparable to our expectations. We will perform a more detailed and precise system calibration after assembling the fully amplified apparatus.

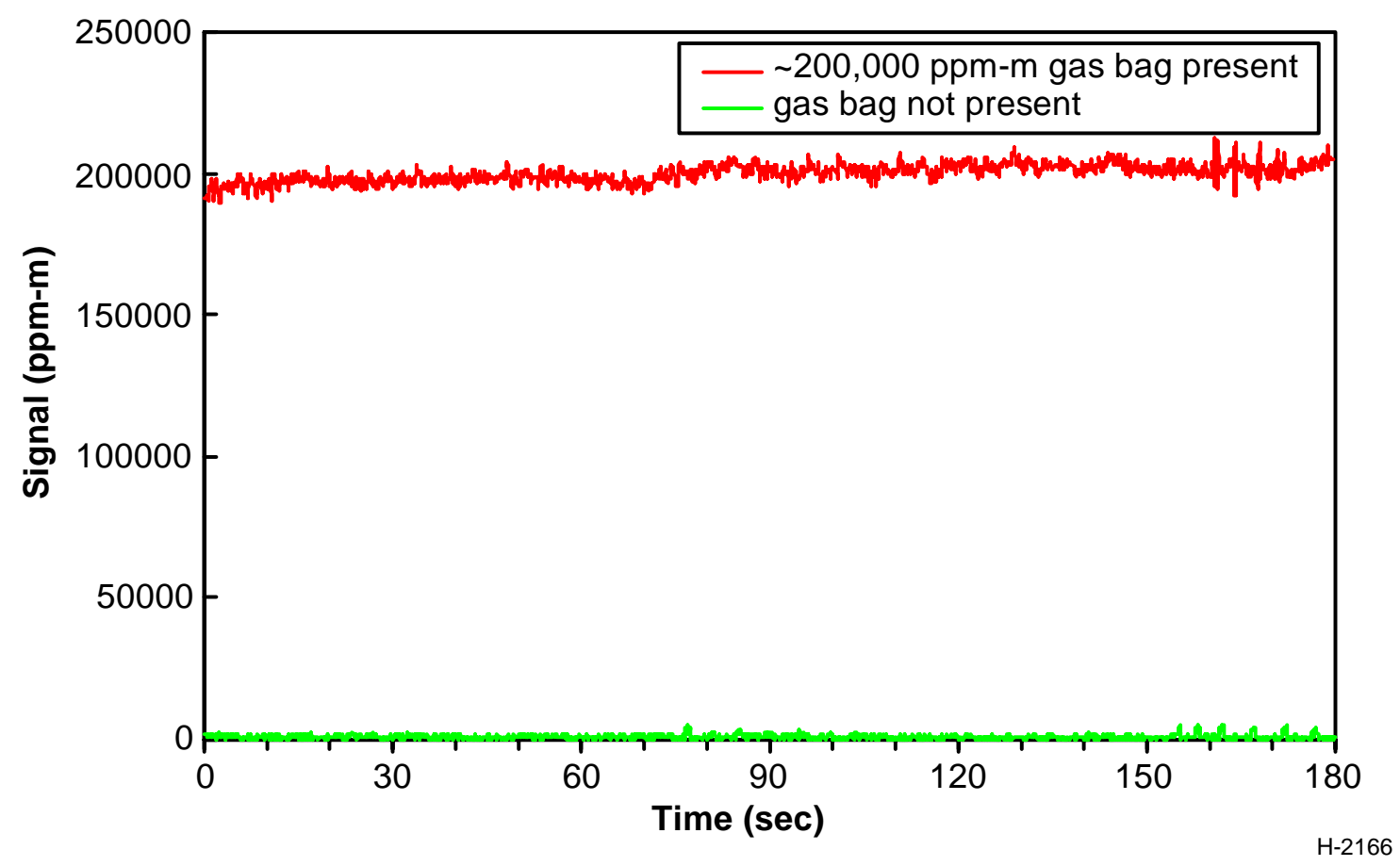

Figure 14. WMS concentration signal for signals received with the unamplified laser and benchtop transceiver from a door at $5 \mathrm{~m}$ distance, with (red)and without (green) an $\sim 10 \mathrm{~cm}$ thick bag of pure methane in the beam path.

Further, and more detailed calibration and characterization, was performed with the unamplified beam directed through the $50 \mathrm{~cm}$ optical cell to a detector at the other end. It should be remembered that a by-product of the wavelength modulation process is a concomitant amplitude modulation of the laser power. As Figure 15 (blue line) illustrates, the amplitudemodulated power transmitted by the seed laser in the absence of target gas in the optical path (cell) is a nearly perfect sinusoid containing no obvious harmonic components. This sinusoid 


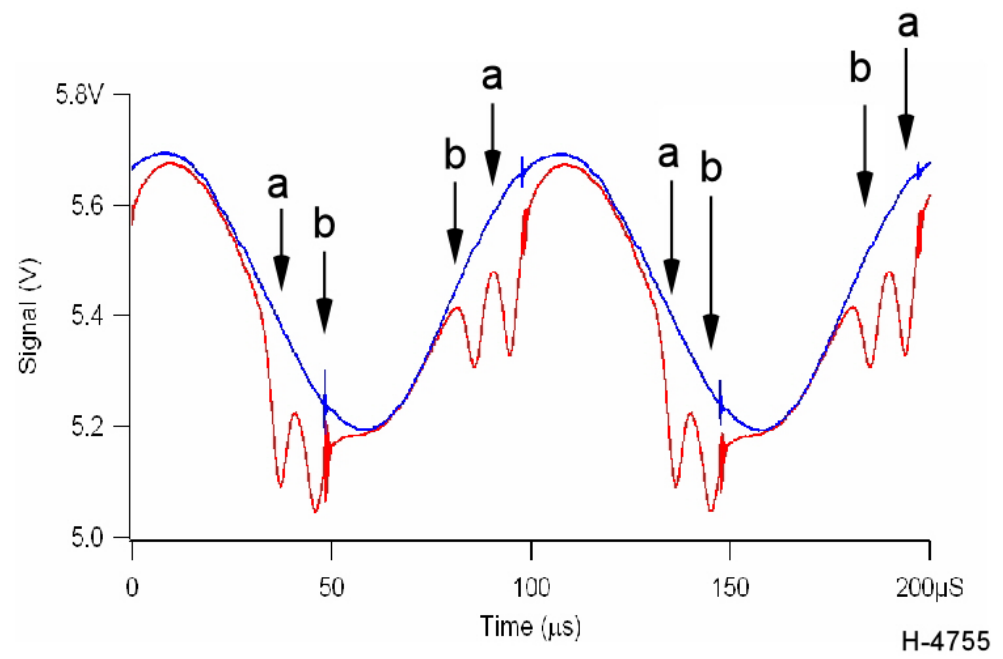

Figure 15. Seed laser power transmitted through $50 \mathrm{~cm}$ cell with (blue) no gas, and (red) 75 torr neat methane. Methane absorption lines, corresponding to those shown in Figure 16, are indicated. $\mathrm{a}=6180.59 \mathrm{~cm}^{-1}, \mathrm{~b}=6180.66 \mathrm{~cm}^{-1}$.

can also be viewed as a measure of laser wavelength versus time. Figure 15 (red line) illustrates the signal received upon transmitting the laser beam through a $50 \mathrm{~cm}$ path of $100 \%$ methane at 75 torr pressure. Absorption by the methane absorption feature diminishes the laser power each time the wavelength crosses it. In comparison, Figure 16 shows the absorbance vs frequency of the $6180.6 \mathrm{~cm}^{-1}$ methane spectral feature, calculated using the HITRAN database. Note that this feature, which appears to be a single line at atmospheric pressure, resolves into a pair of lines at reduced pressure. Figure 17 shows the absorbance versus time deduced from the two curves in Figure 15. The agreement with calculations of Figure 16 is excellent. Figure 18 shows the absorbance versus time with the methane pressure increased to 760 torr (1 atm).

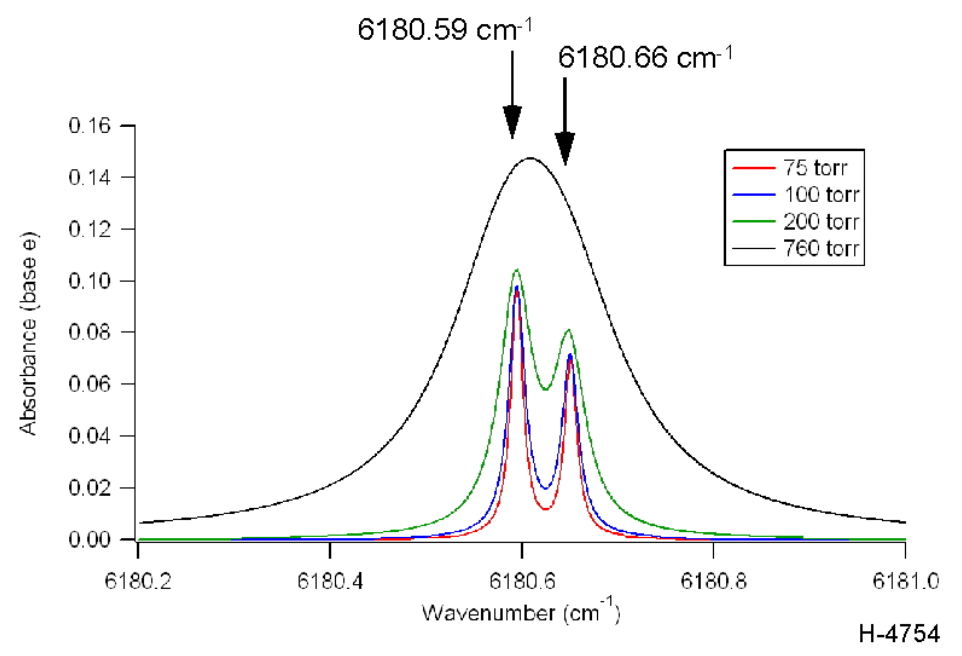

Figure 16. HITRAN-calculated methane spectral feature selected for the high-altitude leak detector at various pressures. Calculation assumes $100 \%$ methane over a $50 \mathrm{~cm}$ path. 


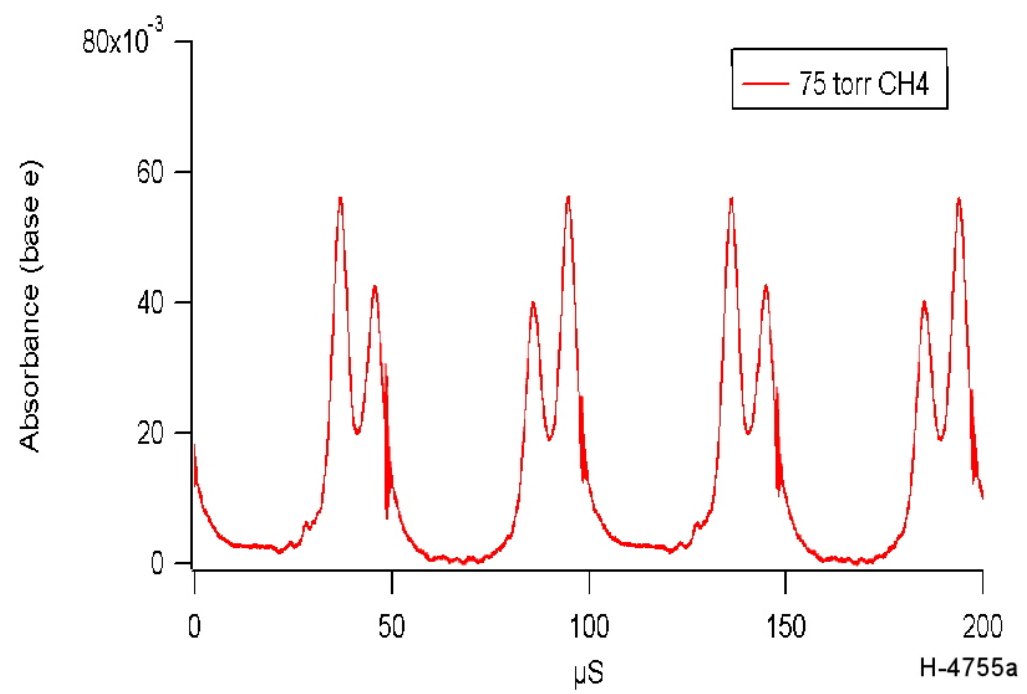

Figure 17. Measured absorbance of seed laser power versus time for 75 torr neat methane in $50 \mathrm{~cm}$ cell. Calculated from raw data shown in Figure 15.

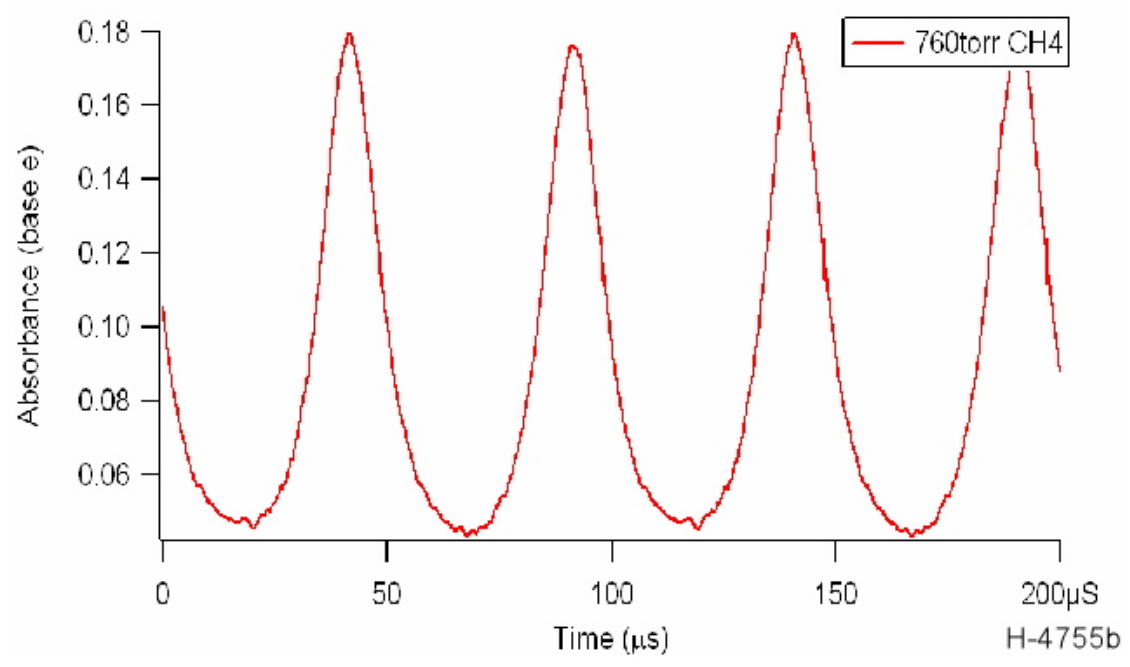

Figure 18. Measured absorbance versus time for 760 torr neat methane in a $50 \mathrm{~cm}$ cell.

Calibration of wavelength versus time during the modulation of the laser can be deduced via a comparison of Figures 16 and 17 or can be measured directly for greater accuracy, especially as the function is not linear. This is achieved by directly illuminating the dectector with the laser beam, as above, but inserting a calibrated etalon in the beam path. Figure 19 illustrates the received signal through a $2 \mathrm{GHz}$ etalon (left) and the fit function of wavelength versus time for the $10 \mathrm{kHz}$ modulation (right). An approximate $0.2 \mathrm{~cm}^{-1}$ wavelength modulation is evident for this $14 \mathrm{~mA}$ modulation of injection current. 

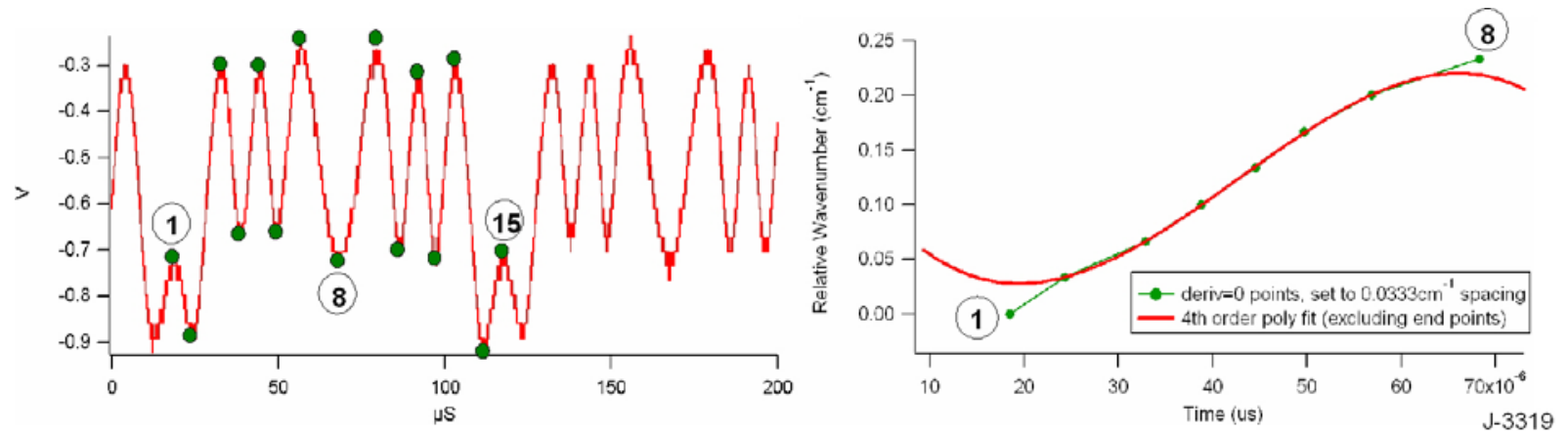

Figure 19. Measured signal from the "seed" laser $(10 \mathrm{kHz}, 14 \mathrm{~mA}$ modulation) through a $2 \mathrm{GHz}$ etalon (left) and the resultant calculation of wavelength modulation (right).

Note that in these WMS signals, the wavelength crosses the methane absorption feature twice for each modulation cycle. Therefore, when methane is in the optical path, the amplitude modulation of the received laser power contains a periodic component having precisely twice the wavelength modulation frequency and fixed in phase relative to the wavelength modulation. In WMS, phase-sensitive (i.e. lock-in) amplification precisely measures the amplitudes of the fundamental sinusoid and this second harmonic component. These are called the $1 \mathrm{f}$ and $2 \mathrm{f}$ signals. The $2 \mathrm{f}$ signal is proportional to the absorbance and thus to the methane concentration, and the $1 \mathrm{f}$ signal is an excellent normalizing signal as it is proportional to total collected laser power (note that the total DC signal at the detector may also be proportional to total returned laser power, but may instead have a contribution from sunlight and also does not have the noiserejecting characteristics of a lock-in signal). Figure 20 shows the $1 \mathrm{f}, 2 \mathrm{f}$, and computed concentration acquired during a ten minute period when transmitting the seed laser beam across the $50 \mathrm{~cm}$ gas cell. During the first 5 minutes, the cell is filled with $100 \%$ methane at $1 \mathrm{~atm}$. The cell is evacuated for the subsequent period. The noise and stability in this configuration meets expectations. The rms deviation during the evacuated period is $\sim 250 \mathrm{ppm}-\mathrm{m}$, which compares favorably with the projected sensitivity of $<1000$ ppm-m.

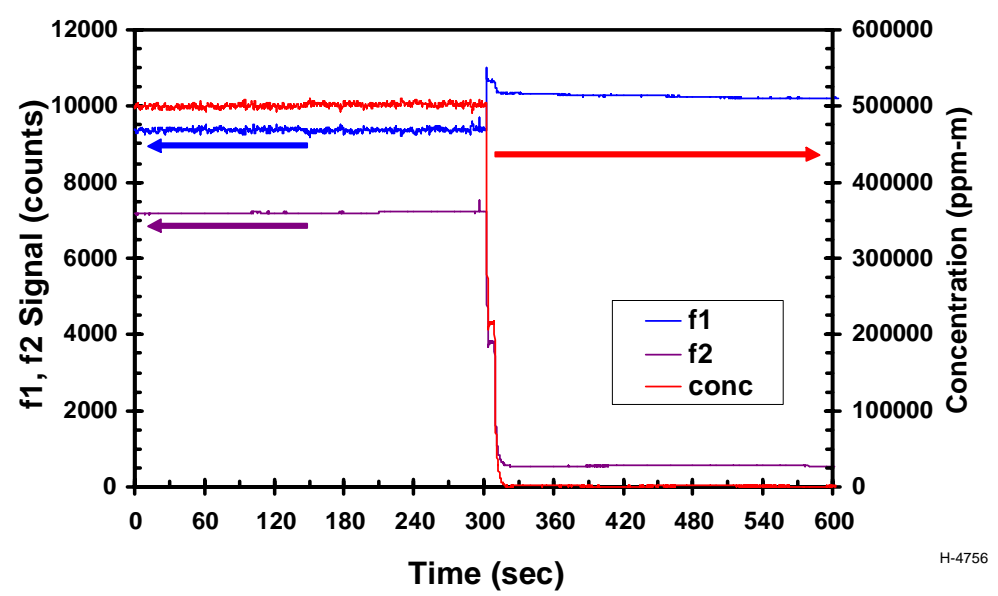

Figure 20. 1f, $2 \mathrm{f}$, and concentration, measured using the seed laser in a $50 \mathrm{~cm}$ cell. Concentration is computed as conc $=(\mathrm{f} 2$ - offset $) / \mathrm{fl} *$ cal, where offset and cal are constants. Actual concentration is $100 \%(500,000 \mathrm{ppm}-\mathrm{m})$ for $\mathrm{t}<300 \mathrm{~s}$, and $0 \%$ for $\mathrm{t}>300 \mathrm{~s}$. 


\section{$\underline{\text { EDFA Characterization }}$}

Gain

The fiber optic laser amplifier is a multi-stage device. The nominal input is $\geq 10 \mathrm{~mW}$ of power from the external seed laser mounted in an RMLD control board. The input then passes through three amplification stages. Each fiber amplifier stage is pumped by a $980 \mathrm{~nm}$ diode laser. The pump laser power for the first stage is fixed, but the user is able to control the pump power, and thus the amplifier gain, for the second two stages. Figure 21 shows the power output by the amplifier as a function of the third stage pump power (represented by the current used to drive the pump laser, which is called Laser Diode 2) for two settings of the second stage pump power (represented as the current driving LD1). The seed laser input for all data in Figure 21 was $18.6 \mathrm{~mW}(12.7 \mathrm{dBm})$, with no modulation, at $1618.0 \mathrm{~nm}$. Figure 21 shows that amplifier output power follows the expected trend: the second stage is essentially saturated and output power is linearly dependent on the gain of the final stage. We had expected the peak output power to be $4.8 \mathrm{~W}$ rather than $3.0 \mathrm{~W}$, but have later deduced this to be an error in calibration of our power meter.

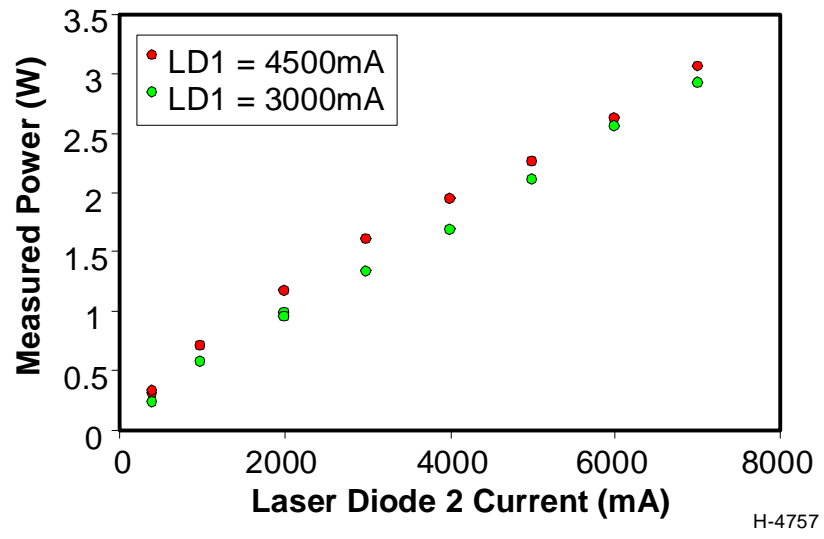

Figure 21. Amplifier output power versus pump laser current settings for fixed seed laser input power of $18.6 \mathrm{~mW}$.

\section{Amplitude Modulation}

Figures 22(a)-(c) show measurements of amplifier output power as functions of time in response to modulation of the seed laser input. These data were acquired by directing the amplifier output beam at a black scattering target and collecting a small amount of the scattered power with a $1 \mathrm{~mm}$ diameter InGaAs detector followed by an electronic pre-amplifier. The preamplifier output was recorded by a digital storage oscilloscope. The oscilloscope was synchronized with the modulation of the seed laser. The seed laser was monitored by using an optical fiber splitter to extract about $5 \%$ of the seed laser power into a second path that terminates at a second detector and pre-amplifier which provides input to a second oscilloscope channel. The seed laser signal is shown as one trace in Figure 22(a). Note that signal magnitude increases in a negative (downward) direction. 


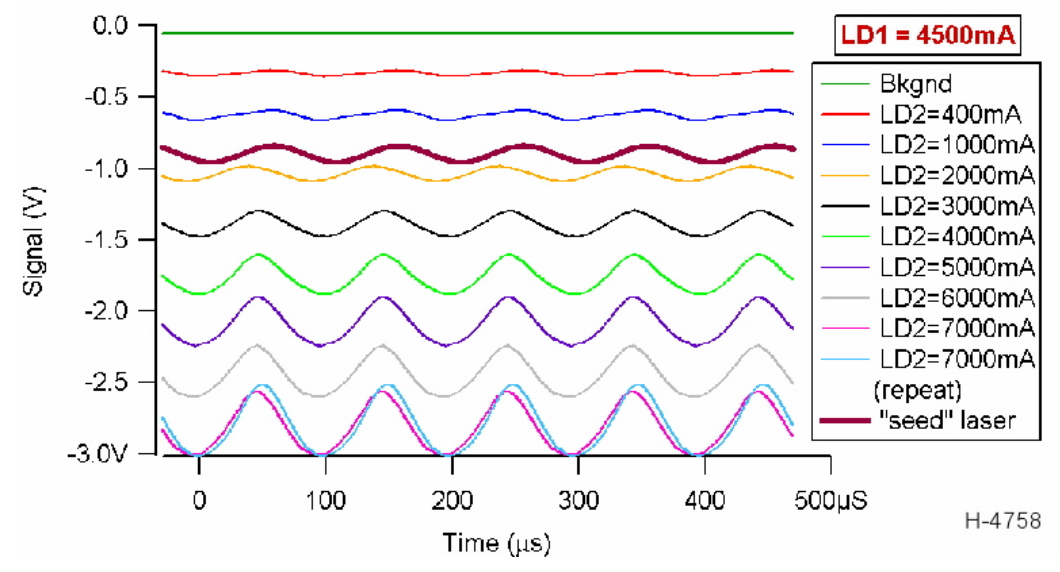

(a)

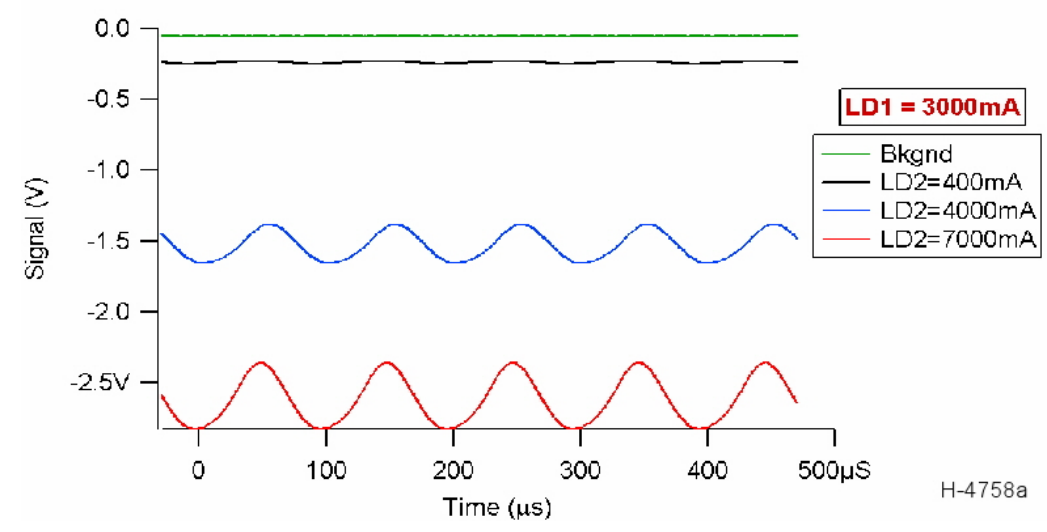

(b)

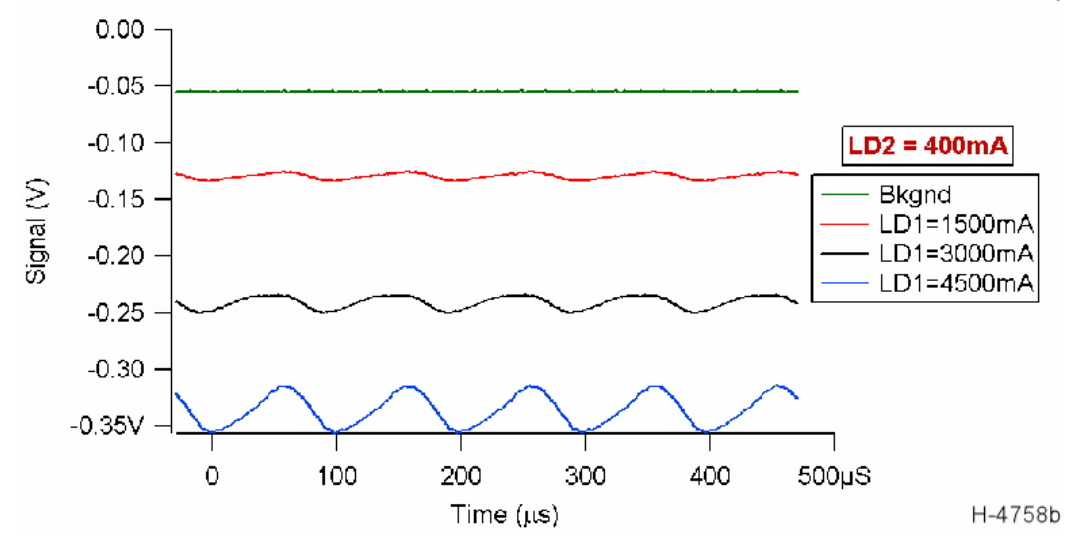

(c)

Figure 22. Laser amplifier output versus pump laser settings during modulated seed laser input. 
The data of Figure 22 reveal several amplifier characteristics: 1) The amplifier output power exhibits amplitude modulation that roughly follows the amplitude modulation of the seed laser. This observation was not previously expected. Indeed, since Figure 21 indicates that the second stage of the amplifier appears to be saturated such that the output of the final stage is nearly independent of second stage gain, we had anticipated that variations of seed laser power would not be transmitted through the amplifier. Furthermore, the gain of the amplitude modulation is approximately the same as the overall amplifier gain. This is illustrated by Figure 23, where we plot the ratio of the modulation amplitude to the average power output from the amplifier, divided by the same ratio for the seed laser. This ratio is close to unity for all operating conditions (except very low gain).

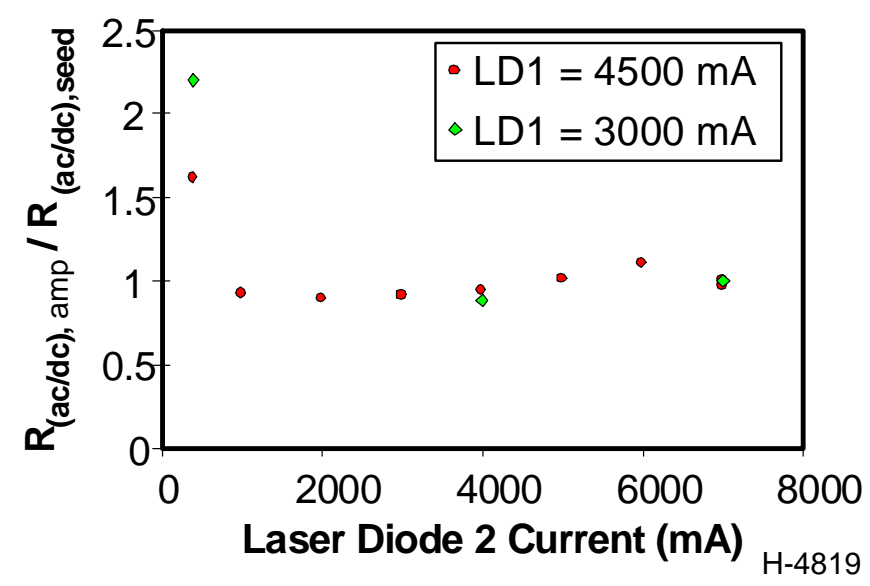

Figure 23. Ratio of modulation amplitude to average power for amplified laser, compared to same ratio for seed laser, as function of amplifier gain.

Although the seed laser amplitude modulation is transmitted by the amplifier, the amplifier output is not a perfect replica of the input. Visual examination of the data in Figure 22 shows that the amplifier output is generally shifted in phase compared to the input, and the output is distorted, i.e., the waveforms are not perfect sinusoids like the input. The implication of this latter observation is that the amplifier output power contains frequency components which can contribute to the $2 \mathrm{f}$ signal utilized to measure methane. If the distortion was invariant over time, then it would contribute a fixed offset to $2 \mathrm{f}$ that could be removed by subtraction. However, the distortion actually varies over time and can therefore be confused with a signal due to methane. The bottom trace in Figure 22(a) shows the amplifier output at maximum gain gathered at two distinct times separated by a few seconds. The deviation from perfect overlap is the indicator that the amplifier distortion varies over time. This variability is quantified in Figure 24, which plots the ratio $2 \mathrm{f} / 1 \mathrm{f}$ for various settings of amplifier gain. We note that $1 \mathrm{f}$, which is a measure of the amplitude modulation at the fundamental frequency, is stable to better than $3 \%$. However, even in the best case (maximum gain), the $2 \mathrm{f}$ signal exhibits drifts, with periods of minutes, having magnitudes that are nearly $20 \%$ of the average value. The implication of this drift for gas measurement will be discussed below, but first we describe the amplifier response to wavelength modulation. 


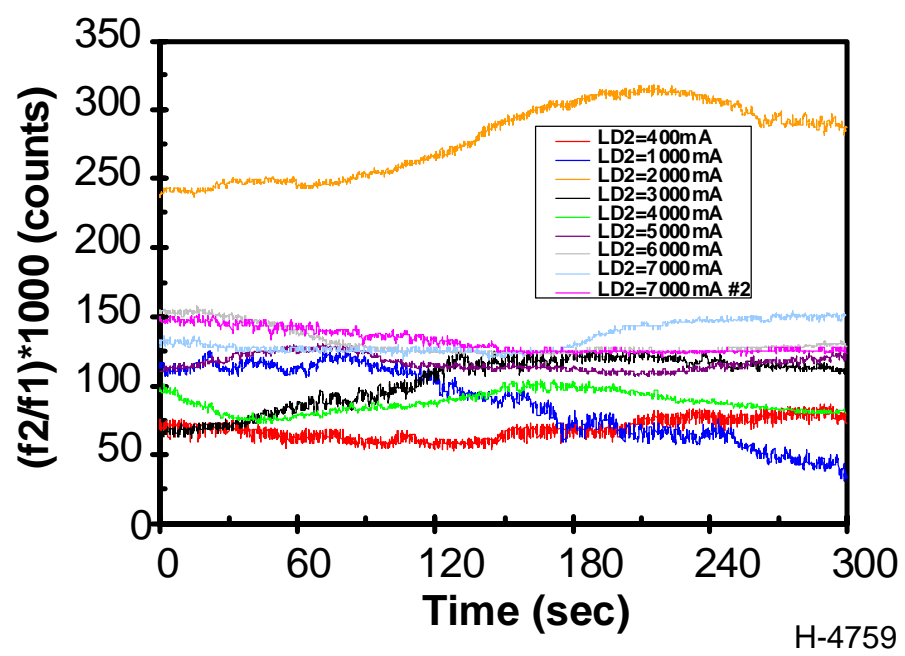

Figure 24. Temporal variation of $2 \mathrm{f} / 1 \mathrm{f}$ resulting from amplitude modulation distortion in the fiber amplifier.

\section{Wavelength Modulation}

Figure 15 presented the power from the seed laser transmitted through a gas sampling cell with and without a 75 torr methane fill, and Figure 17 presented the absorbance calculated from the data of Figure 15. Figures 25 and 26 present similar data, using a portion of the laser beam output from the amplifier rather than the seed laser beam. To gather these data, the amplifier output beam was collimated by a lens and directed at a glass window oriented at a 45 deg angle relative to the beam propagation axis. Approximately 4\% of the laser power reflected from the window surface and was directed into the gas cell; the remainder passed through the window and was collected by an absorber. The portion transmitted through the gas cell was subsequently focused onto the same photodetector and preamplifier combination utilized for the seed laser measurements. As in Figure 15, the absorption by the methane is evident in Figure 25. Indeed, the phase of the absorption signal relative to timing pulses (seen as vertical lines in the signals of Figure 25) is identical to that observed with the seed laser. This indicates that, unlike the amplitude modulation, the wavelength modulation of the amplifier output tracks the seed laser precisely in time. Figure 26 compares the calculated absorbance using the amplified laser beam with that using the seed laser. Again, it is clear that the two signals overlap perfectly in time, but it is also clear that the shape of the absorbance signals using the amplified laser does not smoothly fall to a minimum during the portions of the wavelength modulation when the wavelength is far from the absorption lines. This phenomenon results from the temporal variation of the amplitude modulation distortion.

Figure 27 shows the absorbance with pressure increased to $1 \mathrm{~atm}$. The red curve may be compared with Figure 18. Again, the distortion due to the amplifier is clear. 


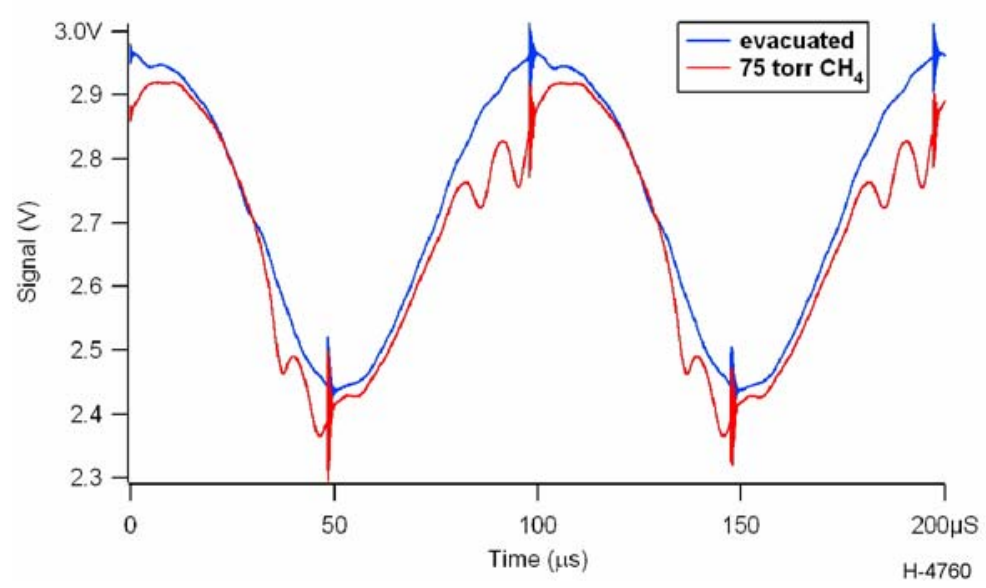

Figure 25. Portion of amplified laser power transmitted through $50 \mathrm{~cm}$ cell with (blue) no gas, and (red) 75 torr neat methane. Compare with Figure 15.

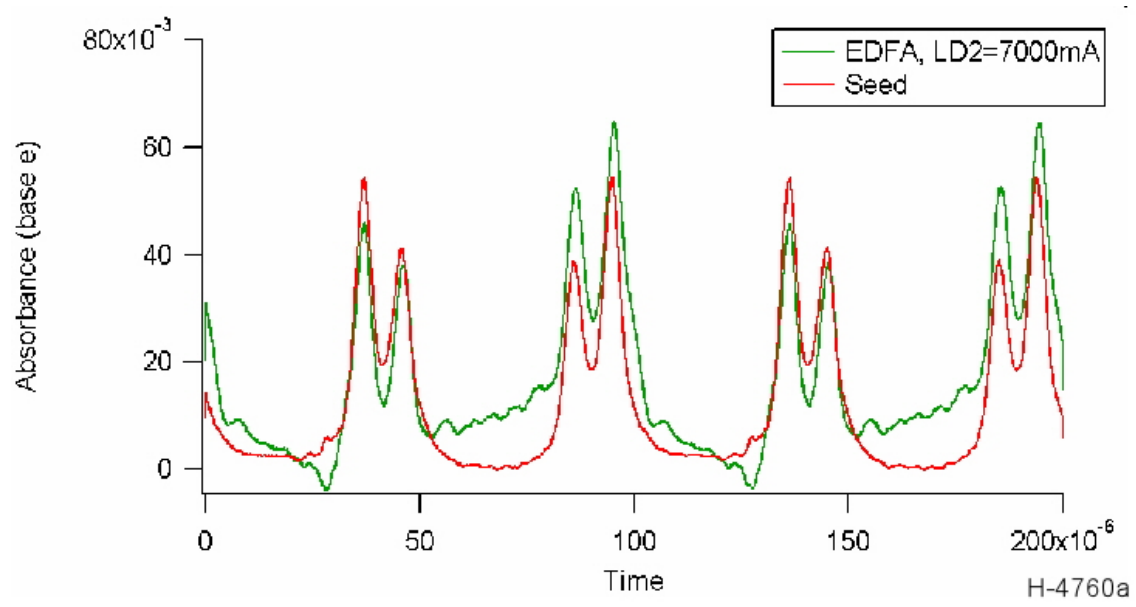

Figure 26. (green) Absorbance of 75 torr neat methane, calculated from data of Figure 25. (red) Overlay of data of Figure 17.

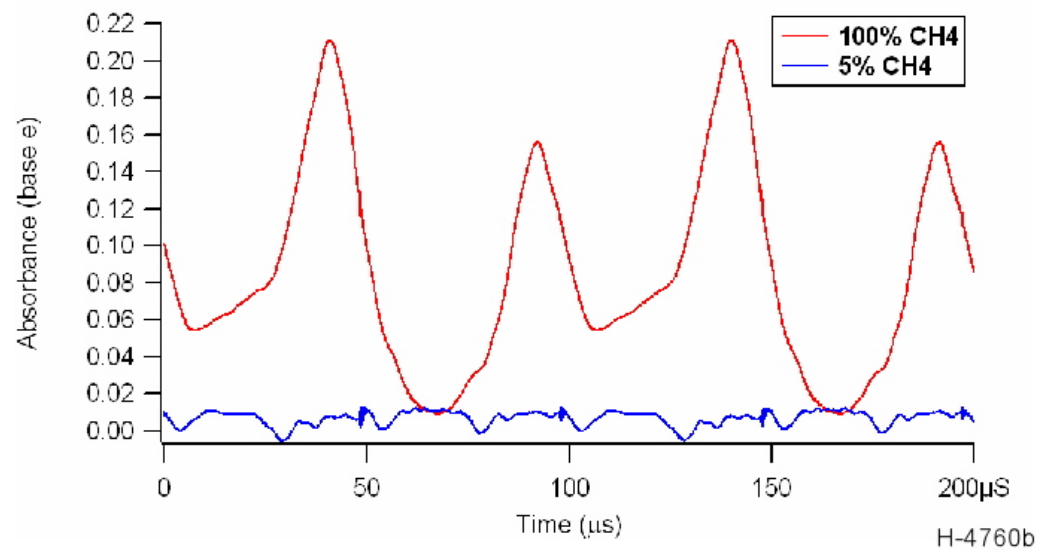

Figure 27. Measured absorbance, using amplified laser, of 760 torr of (red) neat methane and (blue) 5\% methane in $50 \mathrm{~cm}$ cell at 760 torr. Compare with Figure 18. 
Figure 28 plots the 1f, $2 \mathrm{f}$, and concentration values using the amplified laser, first with $50 \%$ methane at 1 atm and subsequently in vacuum. These data may be compared with Figure 20. Note that the rms concentration deviation of the data acquired during the evacuated period is about $15,000 \mathrm{ppm}-\mathrm{m}$. This includes the drift with time scales of minutes plus the random noise. The rms amplitude of the random noise is approximately $5000 \mathrm{ppm}-\mathrm{m}$.

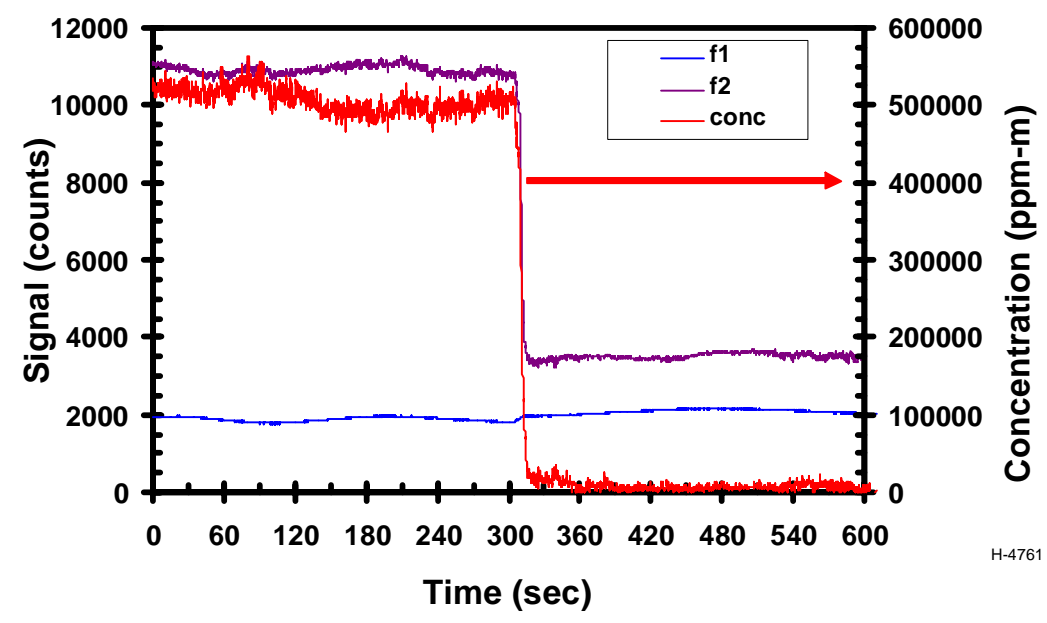

Figure 28. 1f, 2f, and concentration measured using the amplified laser. Compare with Figure 20.

The detection algorithm utilized by the RMLD identifies gas leaks by rapid changes in methane concentration as the laser beam sweeps across a leak plume. These rapid changes occur on time scales of one second or less. Thus, while the relatively slow drift observed with the amplified laser impact the absolute accuracy of measuring methane concentration, it has significantly less impact on the ability to detect a gas leak. Thus, we conclude that gas leaks having plumes that provide a single-pass path-integrated concentration of 2500 ppm-m (half the detection limit of 5000 ppm-m recognizing that the laser beam transits the plume twice on its round trip from and back to the transceiver) can be detected using the amplified laser. This detection limit is roughly one order of magnitude worse than could be achieved if the amplified laser beam achieved the same amplitude modulation characteristic as the seed laser. Nevertheless, we expect that this sensitivity will be adequate to detect the large gas leaks for which the airborne sensor is intended.

\section{Outdoor Ground Field Tests - Assembly and Calibration}

The system was assembled in accordance with the design drawings and is shown (with tripod-mounted transceiver) in the photographs of Figure 29. The tripod, with fine-adjust threeaxis rotation, facilitates aiming at distant objects. The fiber amplifier is mounted on a shockmounted rack installed within a plastic housing suitable for shipping. The entire system is shown mounted in the rear of a box truck, which we have utilized for preliminary outdoor testing. Electrical power for the amplifier is derived from the truck's electrical system. An inverter 

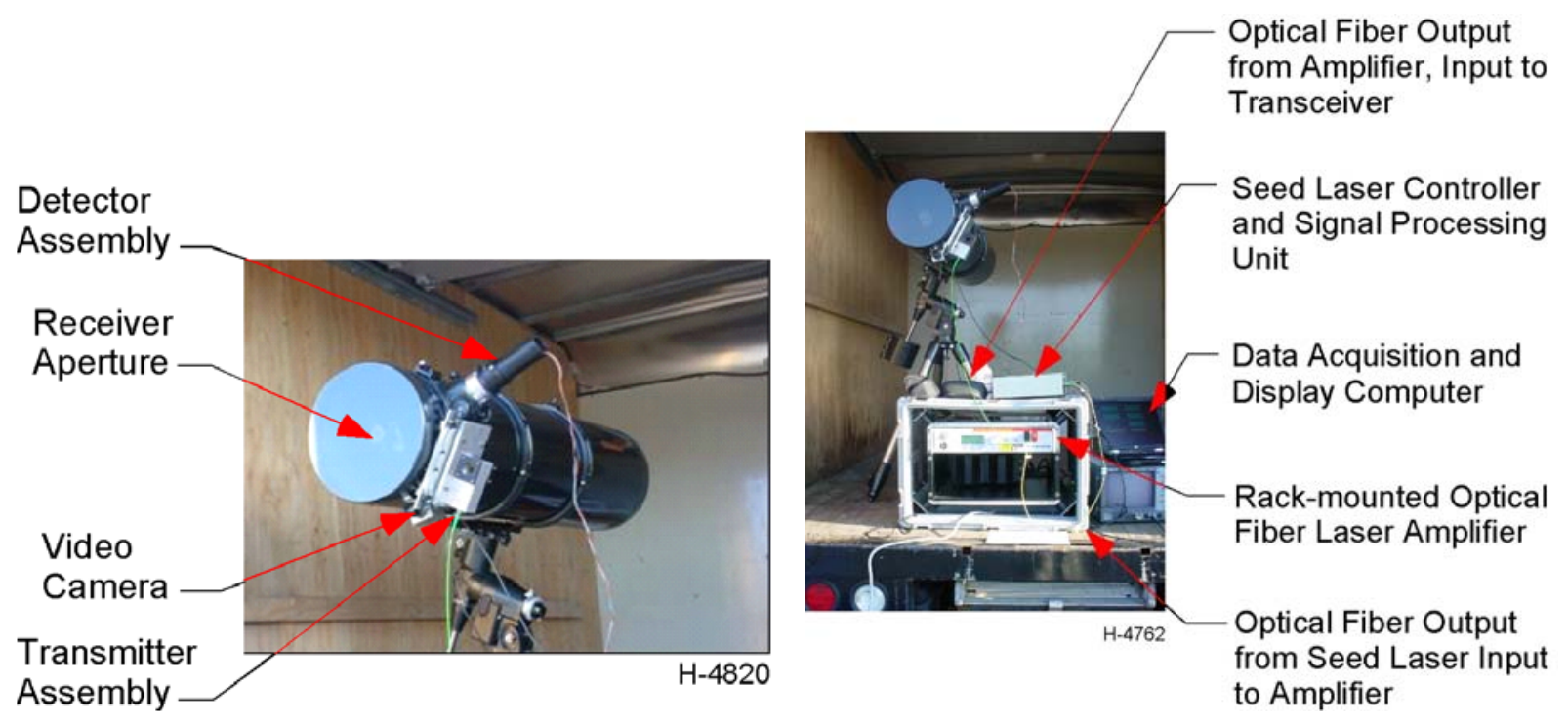

Figure 29. Tripod-mounted transceiver and electronics in box truck.

converts the truck's $12 \mathrm{Vdc}$ supply to $120 \mathrm{Vac}$, and a power supply converts the $120 \mathrm{Vac}$ to the $28 \mathrm{Vdc}$ required by the amplifier. (When utilized on an aircraft, the $28 \mathrm{Vdc}$ power will be provided directly by the aircraft electrical system).

Also evident in Figure 29 is a plastic film secured over the aperture of the telescope. With a gas line plumbed into the body of the telescope, this allowed us to purge the telescope with a calibration gas of known $\mathrm{CH}_{4}$ content and calibrate WMS signals in the outdoor environment. This method was checked against short-range $(\sim 5 \mathrm{~m})$ measurements made in the lab. For these measurements, the laser aim and detector position were optimized for this short range. Figure 30 shows absorbance spectra calculated from signals with and without the calibration gas present in the cell, for both the seed laser and the amplified laser. As the telescope is designed for an infinite conjugate, nearfield collection of photons is inefficient. Thus, the absorbance calculated from the seed laser signals is noisier, as a result of signals that are weak enough to get close to the electronic noise limit. However, both absorbance calculations agree, and the magnitude of

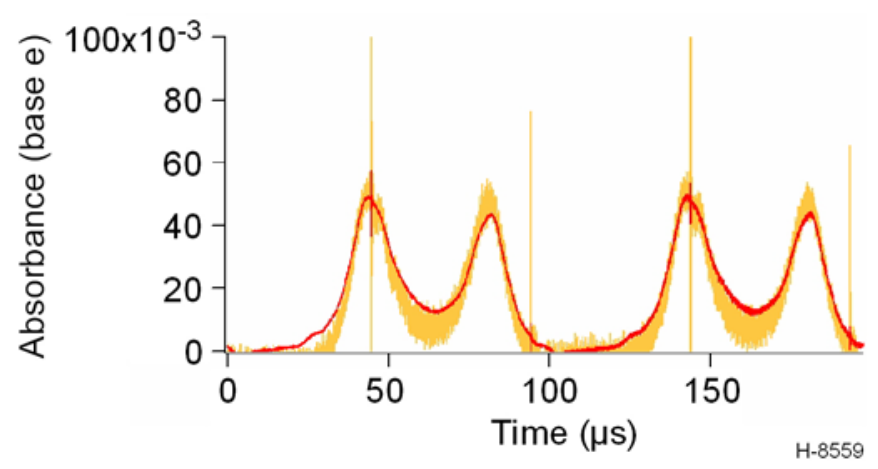

Figure 30. Absorbance generated using the telescope as a calibration cell with $10 \% \mathrm{CH}_{4}$ in $\mathrm{N}_{2}$, for both amplified and unamplified lasers at $\sim 5 \mathrm{~m}$ target range. 
$45 \times 10^{-3}$ corresponds to $126,000 \mathrm{ppm}-\mathrm{m}\left(2.3 \mathrm{~m}\right.$ path, $5.4 \% \mathrm{CH}_{4}$ (gas was shut off before completely purging the telescope)) with an absorption coefficient of $0.36 \mathrm{~atm}^{-1} \mathrm{~m}^{-1}$. WMS signals were recorded simultaneous to these direct signal measurements, also. Again, the observed signal to noise ratio on EDFA-generated signals yields a noise-equivalent $\mathrm{CH}_{4}$ leak sensitivity of 5000ppm-m.

\section{Outdoor Ground Field Tests - Short Range Measurements}

Initial outdoor tests were conducted at a moderate range of $\sim 200 \mathrm{~m}$. In this scenario, the sensor points horizontally across the available extent of an empty lot to a $1 \mathrm{~m} \mathrm{x} 1 \mathrm{~m}$ plywood target. This is somewhat larger than the footprint of the 'collimated' laser beam at this range. One side of this reversible target is bare plywood and the other has retroreflective tape applied. The retroreflective target allows for a strong reflection of a visible alignment laser or the unamplified IR laser. Figure 31 illustrates the CCD camera's view of this target when the telescope has the target in the center of its field of view. As the camera has a wide field of view, it was deemed not necessary to incorporate aim adjustment to put the target in the center of its view. Also, regarding the odd picture angle, the rotational orientation of the telescope (and thus the camera image) is made convenient to the laser launch aim adjustment rather than the camera image. Note that the green circle represents the telescope's ocular field of view (with eyepiece), but the detector field of view is just larger than that of the laser footprint, as shown in Figure 11.

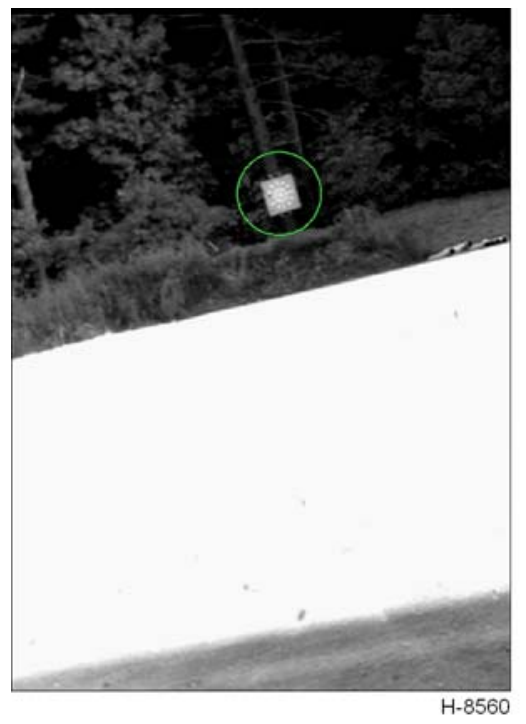

Figure 31. Camera view of plywood target with retroreflective tape at $200 \mathrm{~m}$ range. Telescope ocular field of view is illustrated by the green circle.

After aligning the telescope and the collimated visible laser onto the retroreflective target by eye, fine adjustment to laser aim and detector focal position was achieved by maximizing the detected 1f signal amplitude from the unamplified 'seed' laser reflected by the target. An example of this is evident at the beginning of the time record of WMS signals shown in Figure 32. In this experiment, the sensor is detecting light from the seed laser reflected by the retroreflective target. At $200 \mathrm{sec}$, the calibrated purge gas $\left(10 \% \mathrm{CH}_{4}\right)$ is turned on and begins 


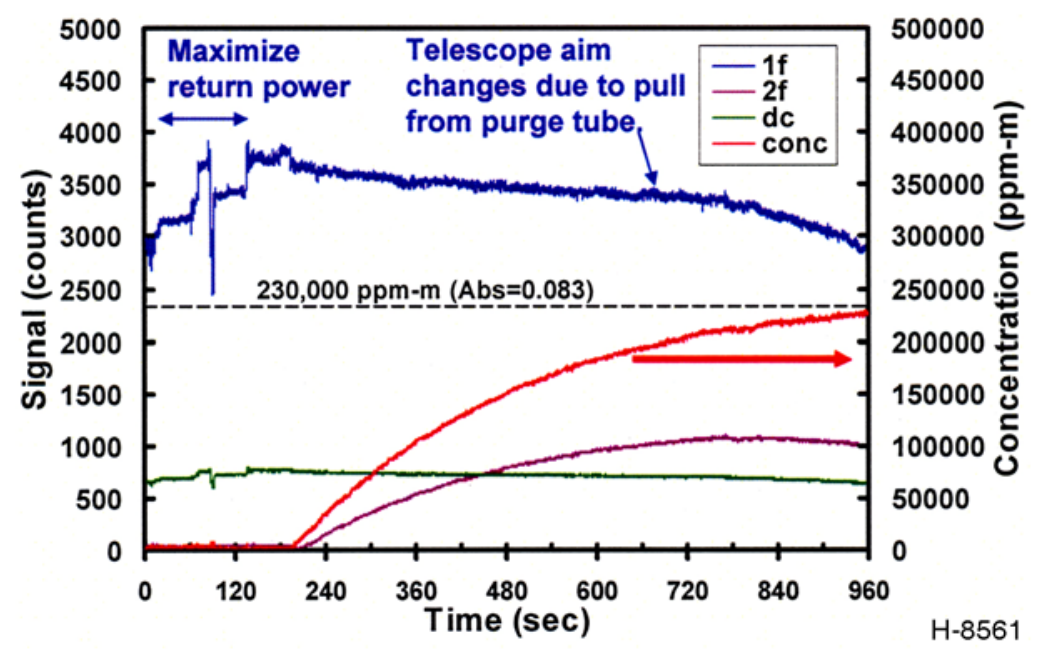

Figure 32. Time record of WMS signals from a retroreflector at $\sim 200 \mathrm{~m}$. At $\sim 200 \mathrm{sec}, 10 \% \mathrm{CH}_{4}$ (balance $\mathrm{N}_{2}$ ) begins flowing into the telescope.

flowing into the telescope body. If the gas were allowed to flow indefinitely, the telescope should contain entirely calibration gas and, given the $2.3 \mathrm{~m}$ optical path inside the telescope, produce a path-integrated concentration of 230,000 ppm-m and an absorbance of 0.083 . Unintentionally, but interestingly illustrated by this experiment, is the insensitivity of the sensor to return power. The telescope was slowly being pulled out of optimal alignment to the retroreflective target by some pressure from the attached purge tube, but the $\mathrm{CH}_{4}(2 \mathrm{f} / 1 \mathrm{f})$ signal is unaffected. Toward the end of this file, when the telescope was $\sim 90 \%$ filled with calibration gas, a calibrating record of direct absorbance on the signal was made. This calculated absorbance and the measured signals that generated it are shown in Figure 33. Note that, as evident in the absorbance calculation, some baseline instability is present in the signal (compare to Figure 18). This is probably due to a drift in a small interfering etalon contribution. Such an effect is not an interference to WMS signals, unless it happens to fall at exactly the $2 \mathrm{f}$ frequency. Baseline aside, the nominal recorded 0.075 absorbance $(=208,000 \mathrm{ppm}-\mathrm{m})$ was matched to the temporally corresponding $2 \mathrm{f} / 1 \mathrm{f}$ counts to produce the calibration utilized in the right-hand "concentration" axis of Figure 32. Similar signals were collected from smaller concentrations (see Figure 34) (abs $=0.045=\sim 125,000 \mathrm{ppm}-\mathrm{m}$ ) and from the bare plywood target. The small baseline drift is still present to different extents, but the calibration of $2 \mathrm{f} / 1 \mathrm{f}$ counts to concentration is consistent with the initial calculation (specifically, this was 626,000 ppm-m per 2f/1f). 

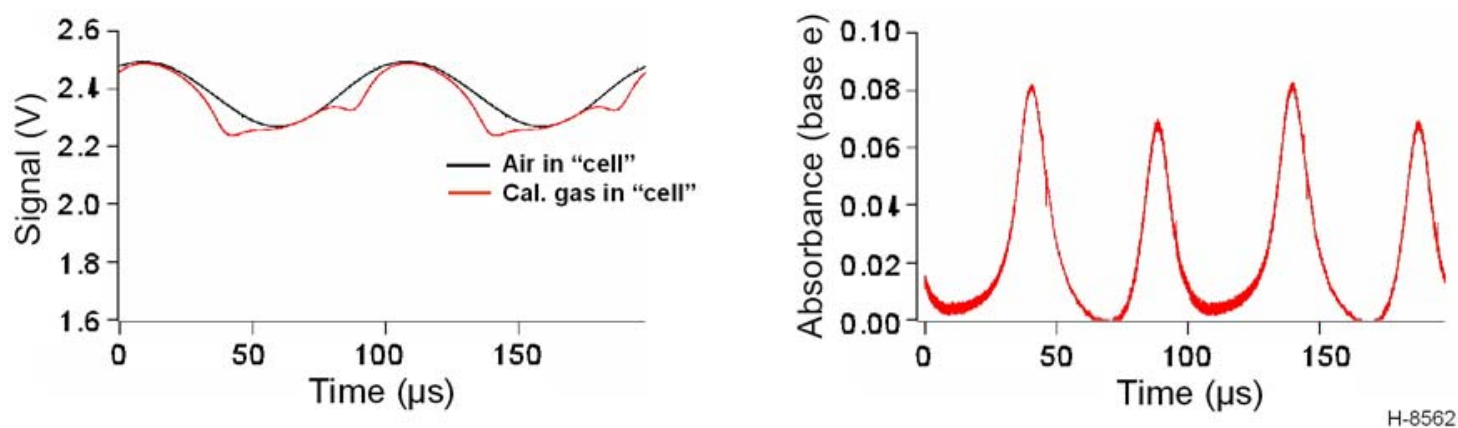

Figure 33. (left) Signals.recorded directly from detector/preamp combo (retroreflector target), including (black) before the $10 \% \mathrm{CH}_{4}$ calibration gas is turned on and (red) at a point when the telescope is nearly filled with the gas (208,000ppm-m). (right) $\mathrm{CH}_{4}$ absorbance calculated from the two recorded signals.
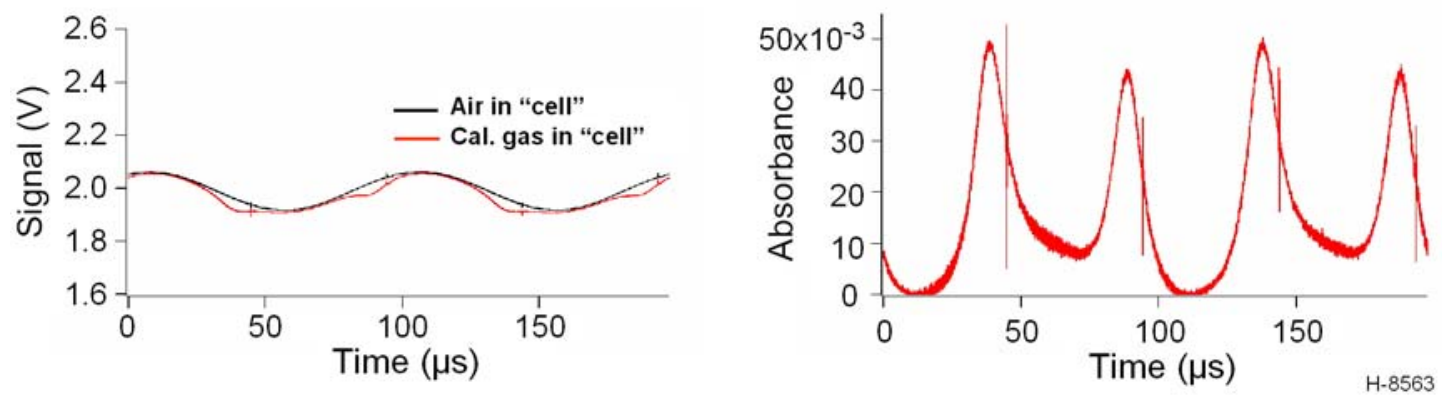

Figure 34. (left) Signals.recorded directly from detector/preamp combo (retroreflector target), including (black) before the $10 \% \mathrm{CH}_{4}$ calibration gas is turned on and (red) at a point when the telescope is partially filled with the gas (133,000ppm-m). (right) $\mathrm{CH}_{4}$ absorbance calculated from the two recorded signals.

The next step was to record baseline signals, again at $200 \mathrm{~m}$, with the amplified laser, and then to measure the response to methane, just as with the seed laser above. Signals were recorded from the amplified beam reflecting off the plywood target. The power in this beam $(>4 \mathrm{~W})$ is more than sufficient to record strong signals from this dull target. As one can see by the $1 \mathrm{f}$ counts in Figure 35, the signal strength of the amplified beam from the plywood target is about twice as strong as that of the seed laser from the retroreflector. In terms of actual received optical power, this is $\sim 1.4 \mu \mathrm{W}$ versus $0.6 \mu \mathrm{W}$. These signals were recorded after about an hour's warm- up time for the EDFA to come to thermal equilibrium after being ramped to full power. At $180 \mathrm{sec}$, the calibration gas is again turned on and begins flowing into the telescope body. In less than 10min, a concentration equilibrium is achieved in the tube. Two points should be noted in regard to the calculation of the concentration signal (red curve): (1) the calculation uses the same calibration constant (ppm-m per $2 \mathrm{f} / 1 \mathrm{f}$ count ratio) as calculated from the seed laser data above, and (2) the $\sim 1000$ counts of $2 \mathrm{f}$ offset (no $\mathrm{CH}_{4}$ present) has been subtracted vectorially from the recorded $2 \mathrm{f}$ quadrature lock-in components, $2 \mathrm{f}(\mathrm{i})$ and $2 \mathrm{f}(\mathrm{q})$. The lock-in signals $2 \mathrm{f}(\mathrm{i})$ and $2 \mathrm{f}(\mathrm{q})$ are the $2 \mathrm{f}$ signal amplitudes detected 'in-phase' with the laser modulation and ' $90^{\circ}$ out of phase' from the 


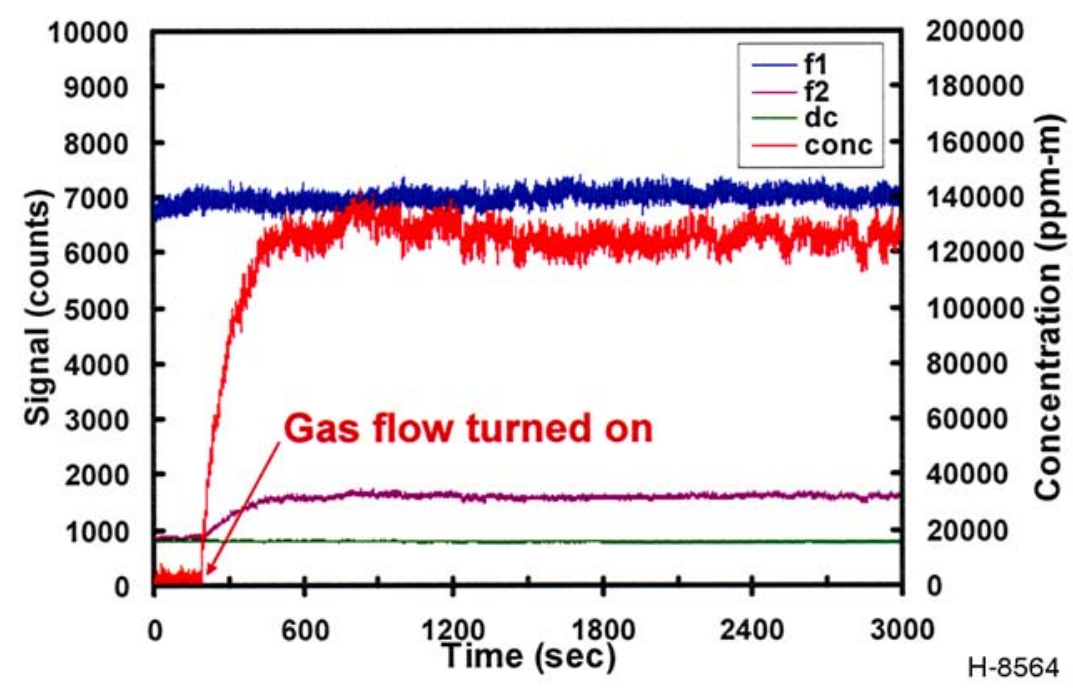

Figure 35. Time record of WMS signals from a plywood target at $\sim 200 \mathrm{~m}$ using the EDFAamplified laser. At $\sim 180 \mathrm{sec}, 10 \% \mathrm{CH}_{4}$ (balance $\mathrm{N}_{2}$ ) begins flowing into the telescope.

laser modulation, respectively. The $\sim 1000$ counts of $2 \mathrm{f}$ magnitude with no $\mathrm{CH}_{4}$ present results from the amplified beam having a waveform somewhat distorted from the input waveform. Unfortunately, this distortion changes in time. Short-term noise (on the order of $1 \mathrm{sec}$ ) on the $2 \mathrm{f} / 1 \mathrm{f}$ offset is $\sim \pm 1 \%$, equivalent to $\sim \pm 2000 \mathrm{ppm}-\mathrm{m}$. Long-term drift (hours to days. See later in this report.) on the $2 \mathrm{f} / 1 \mathrm{f}$ offset is around $\pm 10 \%$, equivalent to $\sim \pm 20,000 \mathrm{ppm}-\mathrm{m}$. The full offset magnitude is on the order of $200,000 \mathrm{ppm}-\mathrm{m}$. These path-integrated concentration equivalences also take into account the fact that lock-in detection of $\mathrm{CH}_{4}$ on the amplified signals is $\sim 2 \mathrm{x}$ less responsive than on the seed laser signals. For example, note that during the period of $\mathrm{CH}_{4}$ equilibrium a $\sim 126,000 \mathrm{ppm}-\mathrm{mm}$ signal is generated using the seed laser-generated calibration. A check on direct absorption signals recorded during this period (see Figure 36) shows that the path-integrated absorbance is $\sim 0.080(=222,000 \mathrm{ppm}-\mathrm{m})$.
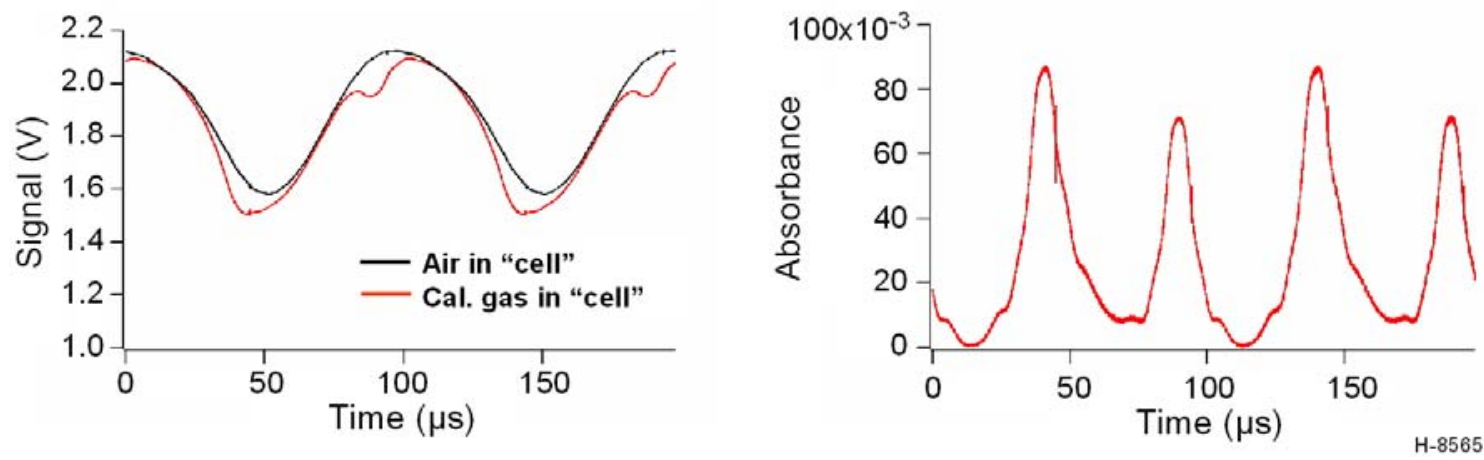

Figure 36. (left) Signals.recorded directly from detector/preamp combo (plywood target) using the EDFA-amplified laser, including (black) before the $10 \% \mathrm{CH}_{4}$ calibration gas is turned on and (red) at a point when the telescope is at an equilibrium concentration of calibration gas $(222,000 \mathrm{ppm}-\mathrm{m})$. (right) $\mathrm{CH}_{4}$ absorbance calculated from the two recorded signals. 
If the phase of this $\sim 200,000 \mathrm{ppm}-\mathrm{m}$ of offset were consistent, one could subtract it from the recorded signal and be left to deal solely with the noise and drift between 2000 and 20,000 ppm-m. In the data above (Fig 35), the phase is known and this offset-correction has been performed. However, the phase of the distortion-induced $2 \mathrm{f}$ slowly drifts, making it difficult to automatically correct for, as it can temporally have lesser or greater contribution to the $\mathrm{CH}_{4}$-induced $2 \mathrm{f}$, which is always at a fixed phase for a particular target distance. The $\mathrm{CH}_{4}$-induced $2 \mathrm{f}$ signal at the $200 \mathrm{~m}$ range, for example, is $\sim 170^{\circ}$ (response mostly in $2 \mathrm{f}(\mathrm{i})$ ). This is evident when looking at the separate $2 \mathrm{f}(\mathrm{i})$ and $2 \mathrm{f}(\mathrm{q})$ components of the above data, as shown in Figure 37 . The phase and relative magnitudes of the offsets (early times) for the unamplified and amplified cases are plainly very different.
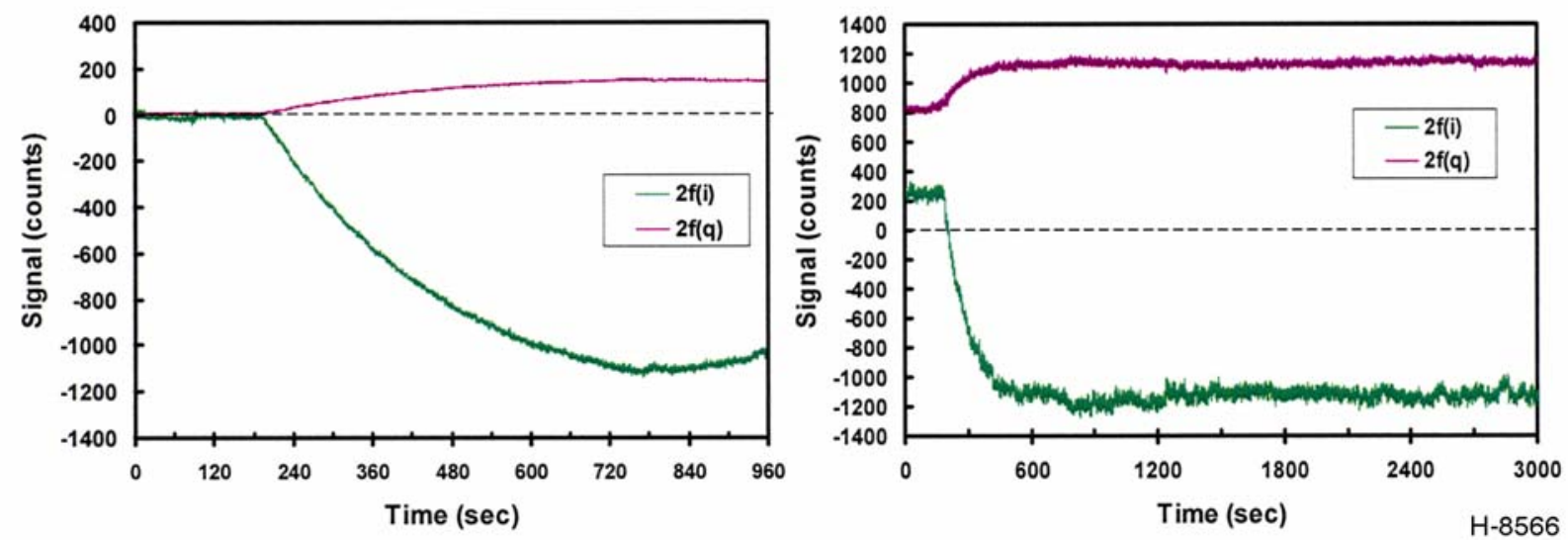

Figure 37. (left) $2 \mathrm{f}(\mathrm{i})$ and $2 \mathrm{f}(\mathrm{q})$ lock-in signals of the data record of Figure $32 . \mathrm{CH}_{4}$ signal occurs at $\sim 170^{\circ}$ from negligible seed laser distortion-induced $2 \mathrm{f}$. (right) $2 \mathrm{f}$ (i) and $2 \mathrm{f}(\mathrm{q})$ lock-in signals of the data record of Figure $35 . \mathrm{CH}_{4}$ signal occurs at $\sim 170^{\circ}$ from EDFA-amplified laser distortion-induced $2 \mathrm{f}$.

The two options available for getting around the unsteady distortion-induced $2 \mathrm{f}$ are: (1) to reduce the distortion, or (2) to try to focus on fast signal changes, i.e. $2 \mathrm{f}$ signal changes on the time scale generated by flying over a pipeline leak. The latter option can be easily adopted, given the RMLD-based leak detection algorithm. The final improvement in sensitivity would be unknown until tested. For signal processing computational simplicity, and uncertainty over leak signal time scales, the former option is preferred. Therefore, an investigation into the characteristics and causes of the distortion on the EDFA-amplified signal was engaged and is described later in this Section.

\section{Outdoor Ground Field Tests - Transient Leak Measurements}

Since putting a transient leak in the sensor's beam path extremely far downfield is problematic, a leak detection experiment was conducted using the $200 \mathrm{~m}$ target range. Also, a since a large leak cloud size is challenging, we aimed for higher concentration in a narrower path to yield the necessary several thousand ppm-m of $\mathrm{CH}_{4}$ required for detection by this sensor. Approximately halfway down the target range a bottle of pure $\mathrm{CH}_{4}$ was valved to release gas very quickly and positioned just under the sensor beam. Figure 38 illustrates several of these gas 


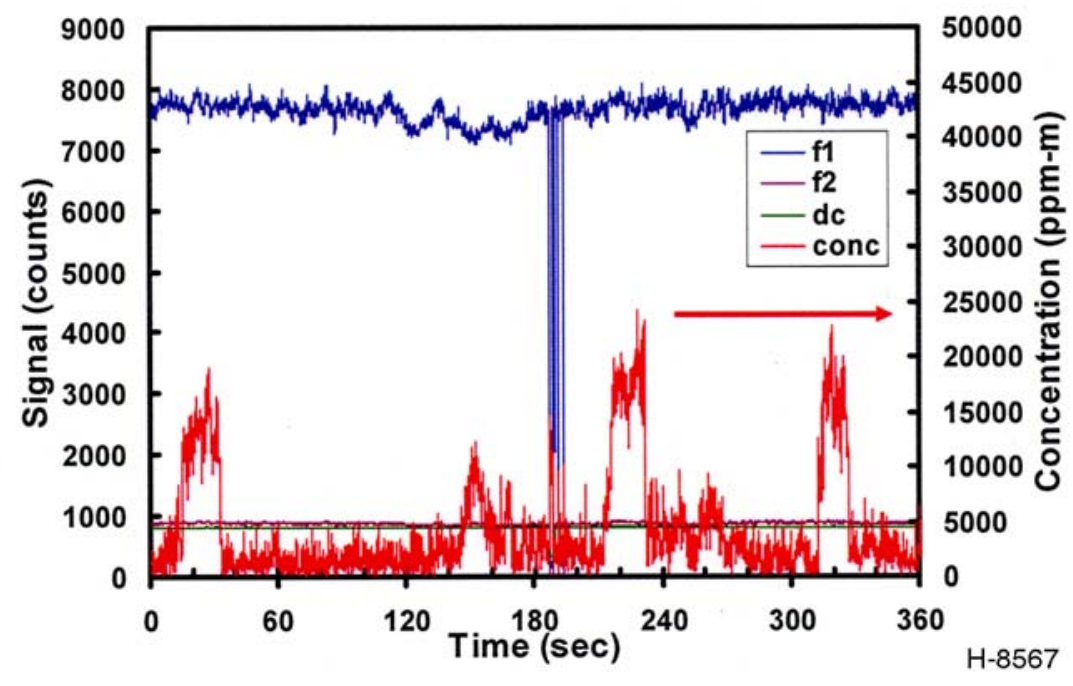

Figure 38. Time record of WMS signals from a plywood target at $\sim 200 \mathrm{~m}$ using the EDFA-amplified laser. At least 4 leak events as $>10,000 \mathrm{ppm}-\mathrm{m}$ are observed.

releases in a logged record of WMS signals generated with the EDFA-amplified beam and the plywood target. The signal dropouts in the middle of the file $(1 \mathrm{f}=0)$ are due to beamfinding at the gas cylinder with a very absorbing beam block. Path-integrated gas concentrations on the order of 20,000ppm-m are apparently all that could be managed with the pointwise gas release. Also, these events were very difficult to observe in real time (having no automatic offset correction). Thus, simultaneous direct absorption signals were not generated, so the events are not independently calibrated. The concentration calculations employ the previously-generated calibration constant. Given the uncalibrated nature of these tests, we performed only a few repetitions. However, the concept is proved that a transient leak larger than the noise-equivalent limit will be detected.

\section{Outdoor Ground Field Tests - Long Range Measurements}

The present prototype of the airborne $\mathrm{CH}_{4}$ sensor was designed to meet the goal of detecting leaks on the order of $1000 \mathrm{ppm}-\mathrm{m}$ at ranges up to $3000 \mathrm{~m}$. Thus, it was deemed prudent to characterize the performance of the sensor with a ground-based target at a comparable distance. This would provide a benchmark performance to compare with airborne sensor signals. Also, at this scale of target distance, ambient background $\mathrm{CH}_{4}$ should be measurable, providing us with an inherent sensitivity test (can we detect the several thousand ppm-m ambient $\mathrm{CH}_{4}$ ?) and a signal contribution that we must learn how to correct for or be insensitive to. The most convenient long-range target in the vicinity of PSI was a water tower at a range of $\sim 6500 \mathrm{ft}$ (near $2000 \mathrm{~m}$ ), visible through a break in foliage, as shown via the sensor camera image in Figure 39. Alignment of the invisible laser on the target is fairly easily performed with the camera. However, confirmation of alignment can be seen in recorded $1 \mathrm{f}$ signals. A fairly stable signal of $\sim 75$ counts is generated as the beam traverses the face of the tower. Figure 40 illustrates this with a record of $1 \mathrm{f}$ signal strength during vertical scans across the tower. This is equivalent to $\sim 15 \mathrm{nW}$ returning to the detector. Thus, in these field measurements, we successfully demonstrated the 


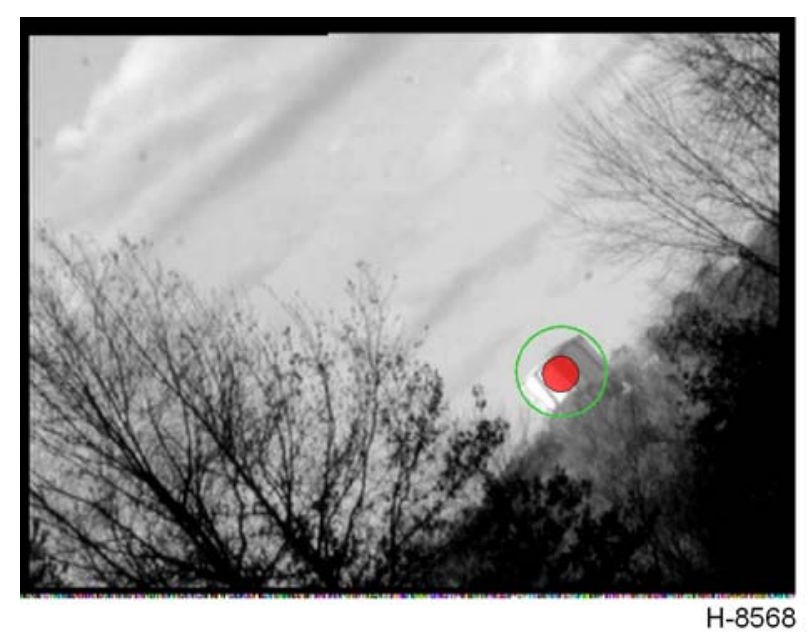

Figure 39. Water tower as viewed by transceiver video camera. The telescope ocular field of view is illustrated by the green circle. The smaller circle is the approximate footprint of the laser beam.

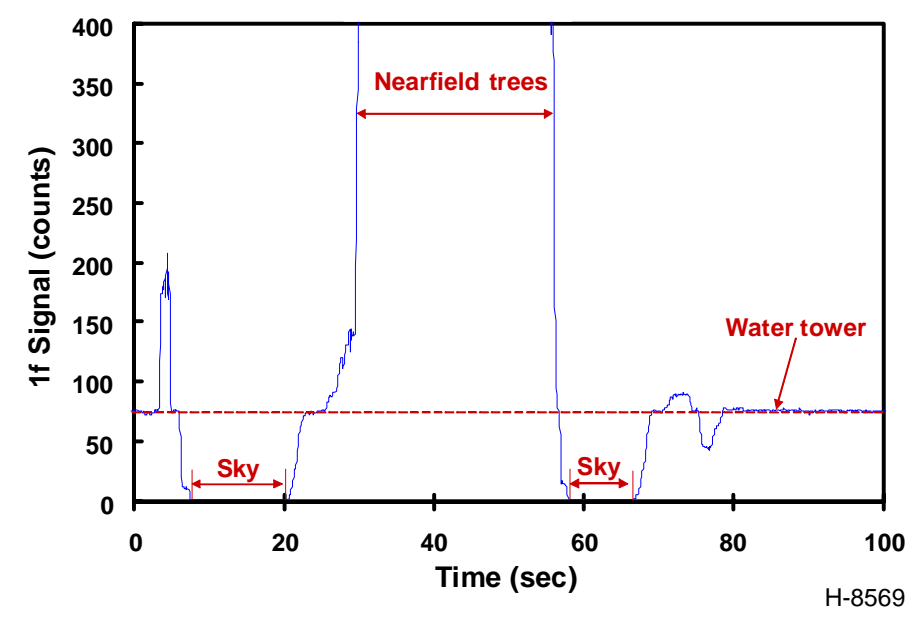

Figure 40. Record of $1 \mathrm{f}$ signals logged while performing vertical scans across the water tower.

ability to transmit and receive sufficient laser power to measure methane from a distance of several thousand meters.

Given the range of $\sim 2000 \mathrm{~m}$ to the water tower, we expected a path-integrated background $\mathrm{CH}_{4}$ contribution on the order of $4000-8000 \mathrm{ppm}-\mathrm{m}$. Also, the round trip distance of $\sim 4000 \mathrm{~m}$ will yield a measurable time lag between laser light leaving the sensor and then being received at the detector. This delay is on the order of $12.5 \mu$ s for this range and will generate an $\sim 90^{\circ}$ phase lag on the $2 \mathrm{f}$ signal $(50 \mu \mathrm{s}$ period). Figure 41 is a time record of WMS signals employing the water tower as the target (note 20x scaling for $2 \mathrm{f}$ ). The left-hand plot is a record of background signals. In the right-hand plot, after $\sim 60 \mathrm{sec}$, the calibration gas is turned on to the telescope. $1 \mathrm{f}$ and $2 \mathrm{f}$ signals are also slightly higher in the right-hand plot due to an improved aim at the water tower. 

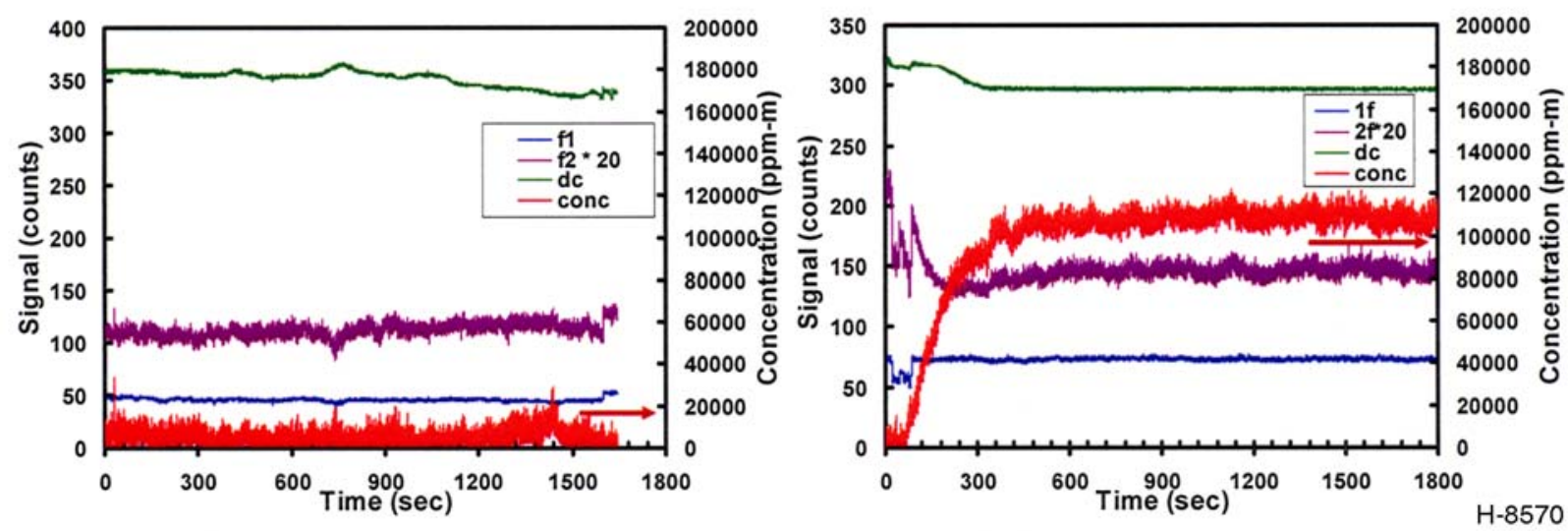

Figure 41. Time record of WMS signals from a water tower at $\sim 2000 \mathrm{~m}$ using the EDFAamplified laser. (left) $\mathrm{No} \mathrm{CH}_{4}$ added. (right) At $\sim 60 \mathrm{sec}, 10 \% \mathrm{CH}_{4}\left(\right.$ balance $\mathrm{N}_{2}$ ) begins flowing into the telescope.

The concentration calculations have again been offset-corrected and employ the seed lasergenerated calibration. Again, also, the sensitivity is $\sim 2 \mathrm{x}$ lower than that of the unamplified seed laser sensor. Assuming 0.080 absorption again, we should see $222,000 \mathrm{ppm}-\mathrm{m}$. Whether the path-integrated $\mathrm{CH}_{4}$ absorbance with a gas-filled telescope is exactly 0.080 again or not could not be validated, as the direct absorption signals were too small and noisy to make the measurement. The calculated noise-equivalent sensitivity $\left(\mathrm{CH}_{4}\right.$ present in "cell" or not present) for a $1 \mathrm{sec}$ time period is $\sim 5000 \mathrm{ppm}-\mathrm{m}$. Drift over the time scale of these records $(\sim 25 \mathrm{~min})$ is about 7000ppm-m.

Figure 42 is the time record of the $2 \mathrm{f}(\mathrm{i})$ and $2 \mathrm{f}(\mathrm{q})$ components of $2 \mathrm{f}$ in the same experiment as Figure 41 (right). Uncorrected signals are on the left, offset-corrected on the right. The $\sim 90^{\circ}$ phase lag for the $\mathrm{CH}_{4}$ signal is most readily seen in the right-hand plot, where the $\mathrm{CH}_{4}-$ induced $2 \mathrm{f}$ signal occurs at $\sim 85^{\circ}$ (mostly $2 \mathrm{f}(\mathrm{q})$ ). Note that since the offset $\left(\sim-55^{\circ}\right)$ is $\sim 140^{\circ}$ out of phase with the $\mathrm{CH}_{4}$ signal, an uncorrected $2 \mathrm{f}$ magnitude gets smaller (closer to zero) with $\mathrm{CH}_{4}$ added to the "cell" (see 2f in Figure 41 (note 20x scaling) and left plot of Figure 42). It is also important to remember that this $2 \mathrm{f}$ offset should, in fact, have some significant ambient $\mathrm{CH}_{4}$ contribution. However, there is no way to decouple what is distortion-induced and what is ambient $\mathrm{CH}_{4}$-induced $2 \mathrm{f}$. Without knowing the distortion contribution magnitude and phase a priori, we cannot conclude whether ambient $\mathrm{CH}_{4}$ has been detected. At this point, we must simply treat ambient $\mathrm{CH}_{4}$ as another potentially-drifting contribution to the $2 \mathrm{f}$ offset (though its phase is known (if target range is known)). We must get around the problem, again, with one of the two methods listed above. 

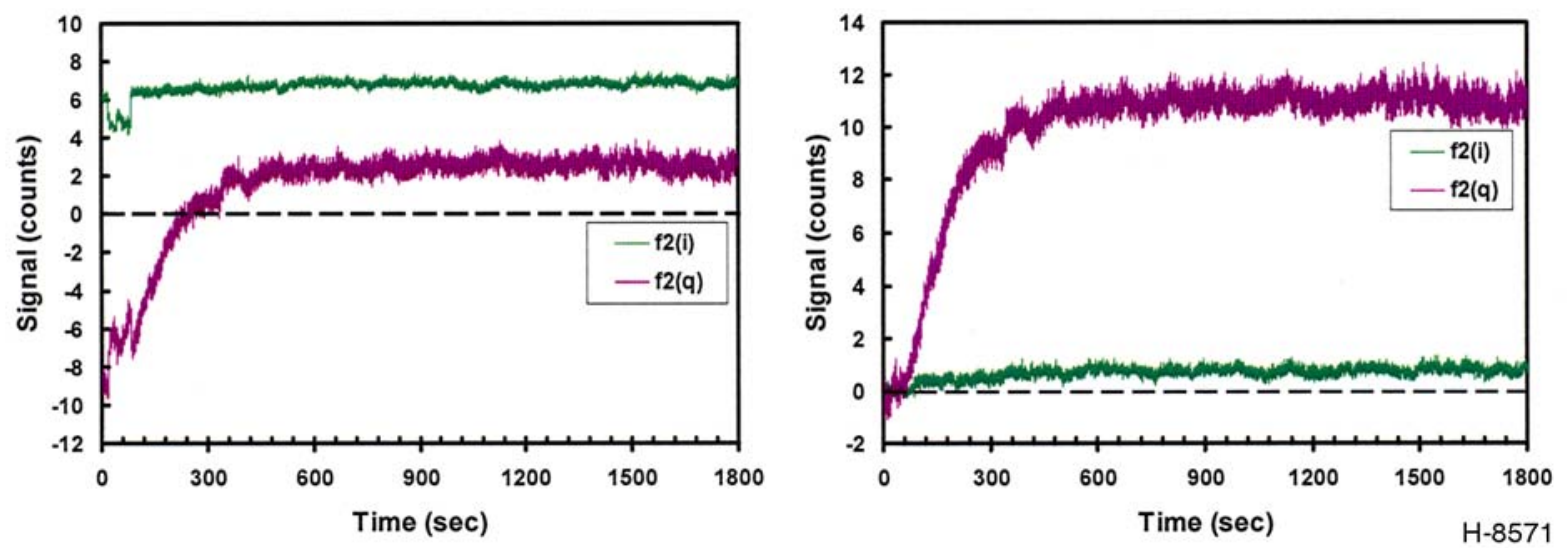

Figure 42. Time record of WMS $2 \mathrm{f}$ quadrature component signals from a water tower at $\sim 2000 \mathrm{~m}$ using the EDFA-amplified laser. At $\sim 60 \mathrm{sec}, 10 \% \mathrm{CH}_{4}\left(\right.$ balance $\left.\mathrm{N}_{2}\right)$ begins flowing into the telescope. (left) Uncorrected (raw) signals. (right) Offset-corrected signals.

\section{$\underline{\text { Long Term Drift }}$}

To attempt to better understand and hopefully improve the performance of the EDFA amplifier, the laser was returned to the benchtop setup for analysis (see Figure 43). The output fiber of the EDFA was still coupled to the OAP mirror launch (removed from telescope), but this beam was directed into a beam dump. A small amount of light was allowed to leak out and be detected by the sensor pre-amp/detector combination (removed from telescope). An initial step was to look into the stability of the phase and magnitude of the distortion-induced $2 \mathrm{f}$ signal. Significantly greater variation in $2 \mathrm{f}$ phase and magnitude had been observed from different experiments day to day than in a single record (up to an hour or so). Movement of input or output fiber was considered as a possible source for this, but observation of signals generated while fibers were intentionally moving showed this not to be the case. Rather, the distortion simply has greater excursions in phase and magnitude over very long (day-scale) time periods. This can be seen in the nearly 3-day time record of WMS signals in Figure 44. Note that the concentration values shown here have not been offset-corrected. The previous seed-laser generated calibration has again been employed. Taking into account the $2 x$ smaller response of the EDFA-generated signals to $\mathrm{CH}_{4}$, the average equivalent offset value over the three days is $187,500 \mathrm{ppm}-\mathrm{m}$. The standard deviation is $15,000 \mathrm{ppm}-\mathrm{m}$. The source of the higher frequency ( $\sim 20 \mathrm{~min}$ periodic) drift is unknown, but we will not investigate it at this point, given the larger scale fluctuations.

We recognize the underlying cause of the drift as due to distortion by the EDFA of the sinusoidal amplitude modulation imposed on the seed laser that is input to the EDFA. The distortion creates a $2 \mathrm{f}$ component which is interpreted as methane in the optical path. If this signal was constant over time, it could be measured once and subtracted as an offset from subsequent measurements. But its temporal variation precludes such a correction. 


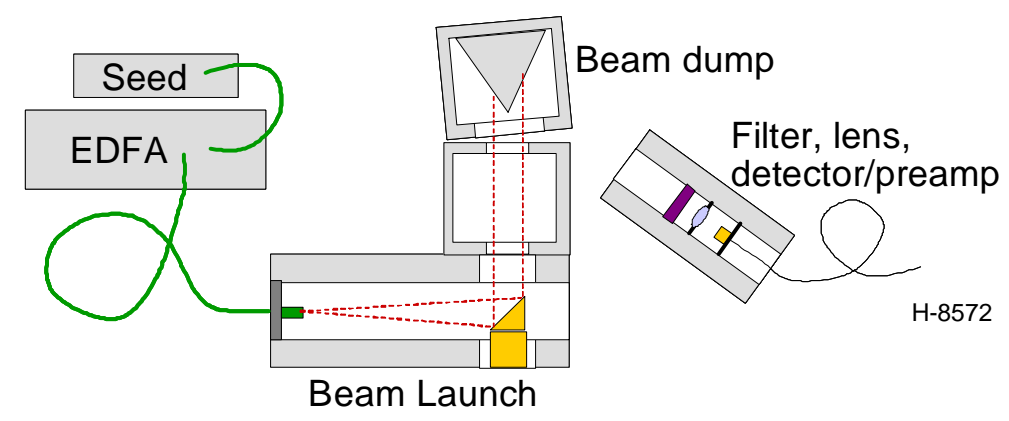

Figure 43. Benchtop experimental setup for laboratory EDFA distortion analysis.
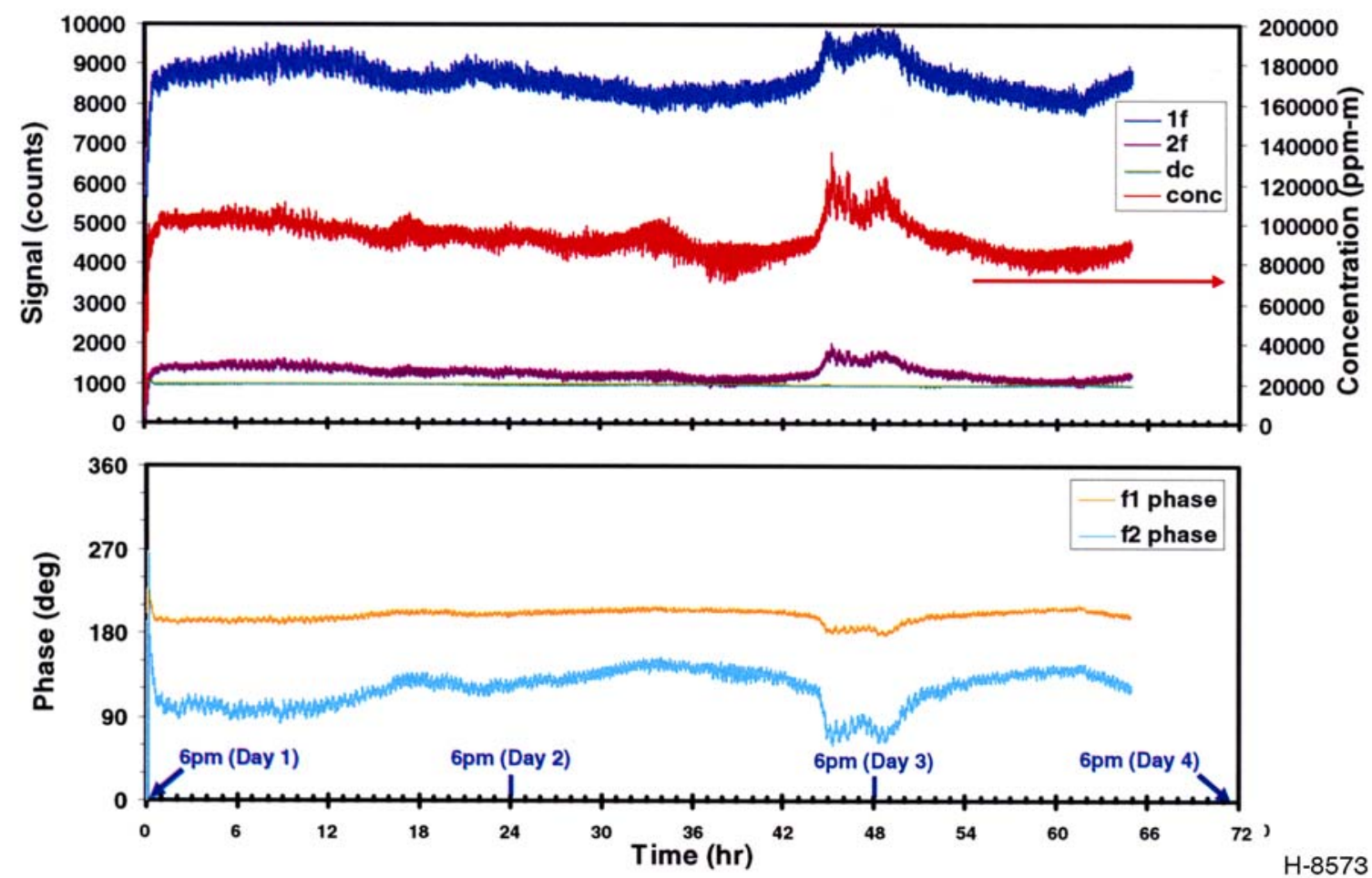

Figure 44. 65-hour time record of WMS signals from a beam dump employing the EDFAamplified laser. (top) Standard signal magnitudes and calculated concentration. (bottom) Phase calculated from recorded 1f(i), 1f(q) and 2f(i), $2 \mathrm{f}(\mathrm{q})$.

Interestingly, though, from these and other signals, it can be seen that there is some correlation between the drift in $1 \mathrm{f}$ and $2 \mathrm{f}$ signals, both magnitude and phase. However, $2 \mathrm{f}$ amplitude fluctuations $( \pm 20 \%)$ are larger than those of the $1 \mathrm{f}$ signal $( \pm 7 \%)$, resulting in a residual drift of $2 \mathrm{f} / 1 \mathrm{f}$ on the order of $\pm 10 \%$. The larger drift of $2 \mathrm{f}$ phase compared to $1 \mathrm{f}$ is even more pronounced. Distortion-induced $2 \mathrm{f}$ phase in these experiments has thus far been observed everywhere between $30^{\circ}$ and $180^{\circ}$. These results were deemed possibly worth revisiting, depending on the success of other analyses. It was conceived that the correlation could be helpful in an attempt to mathematically correct for the distortion-induced $2 \mathrm{f}$. 


\section{EDFA Distortion Characterization}

In an attempt to better understand and mitigate the source of drift., we attempted to characterize certain dependencies of the distortion of the EDFA's output. The EDFA manufacturer (Keopsys), when asked, described three potential sources of amplifier distortion and drift, and some recommendations for characterizing and potentially reducing these effects. They are (1) Amplifier dynamic response, (2) Amplifier gain nonuniformity, and (3) fiber temperature drift effects. This last effect is basically not under user control and is limited by the stability of the temperature control system of the EDFA electronic package. Changes in fiber temperature affect gain curve, metastable state lifetime, and fraction of laser power carried in core vs clad. All of these affect output response to input modulations.

\section{EDFA Dynamic Response}

The amplifier dynamic response can act much like an electronic filter with a time constant determined by the erbium metastable state lifetime. When input seed laser power changes, there is a potentially noticeable lag in amplifier response due to time needed to excite or de-excite erbium atoms. Thus, a sinsoidally modulated input amplitude can have an output modulation at the same fundamental frequency. The amplitude and phase of the output modulation will depend on the input modulation frequency. Since the metastable state lifetime is some fraction of a millisecond, the RMLD's $10 \mathrm{kHz}$ modulation may be a particularly bad operating point. Also of note, as input power changes, the ratio of amplified laser power to amplified stimulated emission (ASE) also changes. Thus, our measure of total output power vs time may not be an accurate representation of power at the seed laser wavelength vs time. Therefore, it was recommended that we map output modulation amplitude vs modulation frequency and consider changing modulation frequency based on the results. The expectation was that higher frequencies would be better.

For these experiments, we built a laboratory apparatus that enabled us to perform Wavelength Modulation Spectroscopy at modulation frequencies ranging from $1-100,000 \mathrm{~Hz}$. As the singleboard WMS laser drive and signal processor is currently designed to run at $10 \mathrm{KHz}$ only, this required the use of a separate benchtop laser drive, lock-in detector, and analog-to-digital signal processor. Figure 45 compares seed laser power input waveforms (oscilloscope records) with EDFA output waveforms for modulation frequencies ranging from $20 \mathrm{~Hz}$ to $100,000 \mathrm{~Hz}$. Note that, previously, it was demonstrated that the EDFA output wavelength is identical to the input wavelength. Therefore, because the seed laser wavelength varies linearly with power, the plot of seed laser power vs time is also a plot of both seed and amplified laser wavelength vs time. The data of Figure 45 confirm that the EDFA distorts the input waveform and that the harmonic content of the distortion depends on the modulation frequency, notably that: 

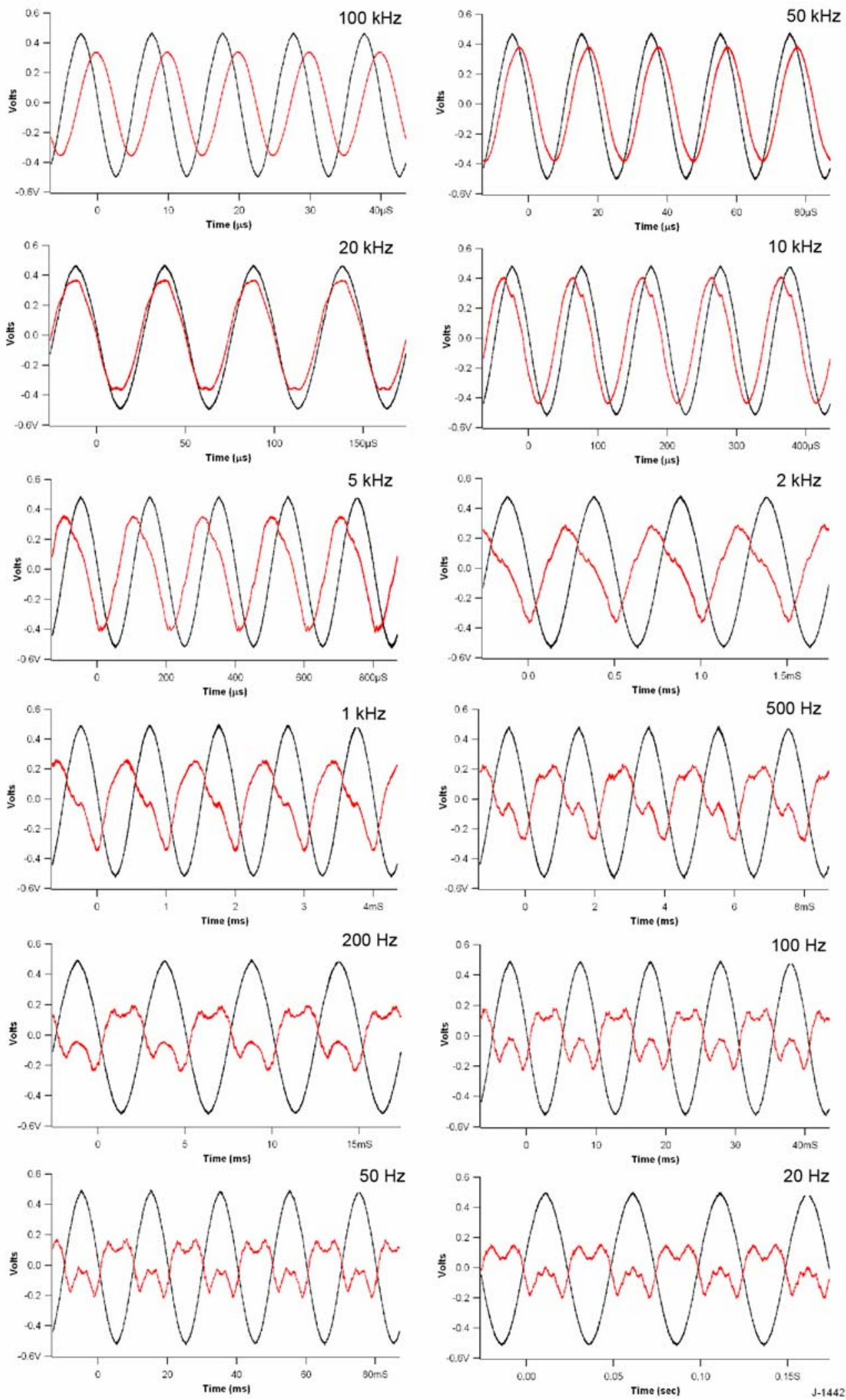

Figure 45. Seed layer (black) and amplified laser (red) power waveforms at modulation frequencies from 20 to $100,000 \mathrm{~Hz}$. 
- At low modulation frequencies $(<100 \mathrm{~Hz})$, the EDFA output power clearly varies nonlinearly with input wavelength. This non-linear response creates a second harmonic (i.e. $2 \mathrm{f}$ ) contribution to the amplified laser waveform. At the low frequencies, this $2 \mathrm{f}$ contribution corresponds to a concentration offset that exceeds $10^{6} \mathrm{ppm}-\mathrm{m}$.

- As modulation frequency increases, the amplitude of the EDFA output at the modulation frequency (i.e. the $1 \mathrm{f}$ signal) increases while the distortion diminishes. At $100 \mathrm{kHz}$, distortion is no longer obvious and the EDFA output power appears to be an undistorted sinusoid that follows the seed laser with a $\sim 90^{\circ}$ phase lag.

The first observation is analyzed further below. The latter observation suggests that operating the EDFA at $100 \mathrm{kHz}$ modulation frequency will yield less distortion-induced drift than operation at lower frequencies. To test this hypothesis, we used a pair of lock-in amplifiers to demodulate the detector output signals at $100 \mathrm{kHz}$ and $200 \mathrm{kHz}$, yielding the $1 \mathrm{f}$ and $2 \mathrm{f}$ signals vs time. Figure 46 illustrates the results. It shows the $1 \mathrm{f}, 2 \mathrm{f}$, and $2 \mathrm{f} / 1 \mathrm{f}$ (proportional to output concentration value) data vs time for a period of nearly two hours. These data were acquired with the amplified laser beam transmitted through a $50 \mathrm{~cm}$ long optical cell. Initially, the cell is filled with only room air. At 950s. the valve to the methane supply was opened briefly, and at 1100 s the cell was evacuated. At 1500s the cell was filled with neat methane, yielding a pathintegrated concentration of 500,000 ppm- $\mathrm{m}$. Then the cell was closed and monitored. During the monitoring period (which lasted about 8 hours), the measured concentration drifted by $23,600 \mathrm{ppm}-\mathrm{m}$, while the noise (defined as rms deviation of individual samples around a 1s average) is about $\sim 4500 \mathrm{ppm}-\mathrm{m}$. These values are comparable to those measured at the $10 \mathrm{kHz}$ modulation frequency. Thus, we conclude that, while modulation at $100 \mathrm{kHz}$ provides less distortion and thus a smaller offset than modulation at $10 \mathrm{kHz}$, the offset drift and noise at the two modulation frequencies are comparable. Thus, there is no practical advantage of the higher modulation frequency.

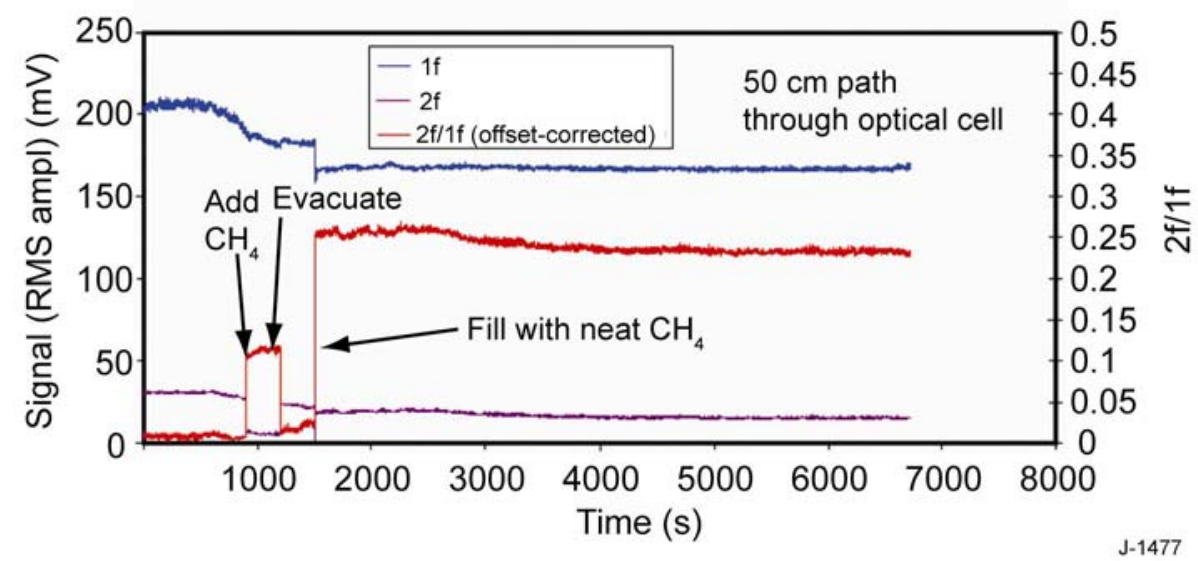

Figure 46. WMS signals recorded at $100 \mathrm{kHz}$ modulation frequency. 


\section{EDFA Gain Nonuniformity}

The dynamic response data above suggested that the amplifier does not have a sufficiently flat (saturated) gain curve across the $\sim 0.2 \mathrm{~cm}^{-1}$ wavelength space that the seed laser scans. This is most evident at lower modulation speeds, where residence times at any particular wavelength are sufficient to deplete the gain medium. A more detailed picture of the nonuniformity of the gain medium can be seen by varying the input wavelength at effectively "dc" rates. Figure 47 illustrates the detected output power from the amplifier as the input wavelength is slowly tuned either by seed laser injection current or temperature tuning. A repeatable non-flat gain curve can be seen that varies by $\sim \pm 4 \%$. This nonuniformity produces distortions in the slower modulated waveforms and yields slowly time-varying offset contributions to the measured WMS signals.
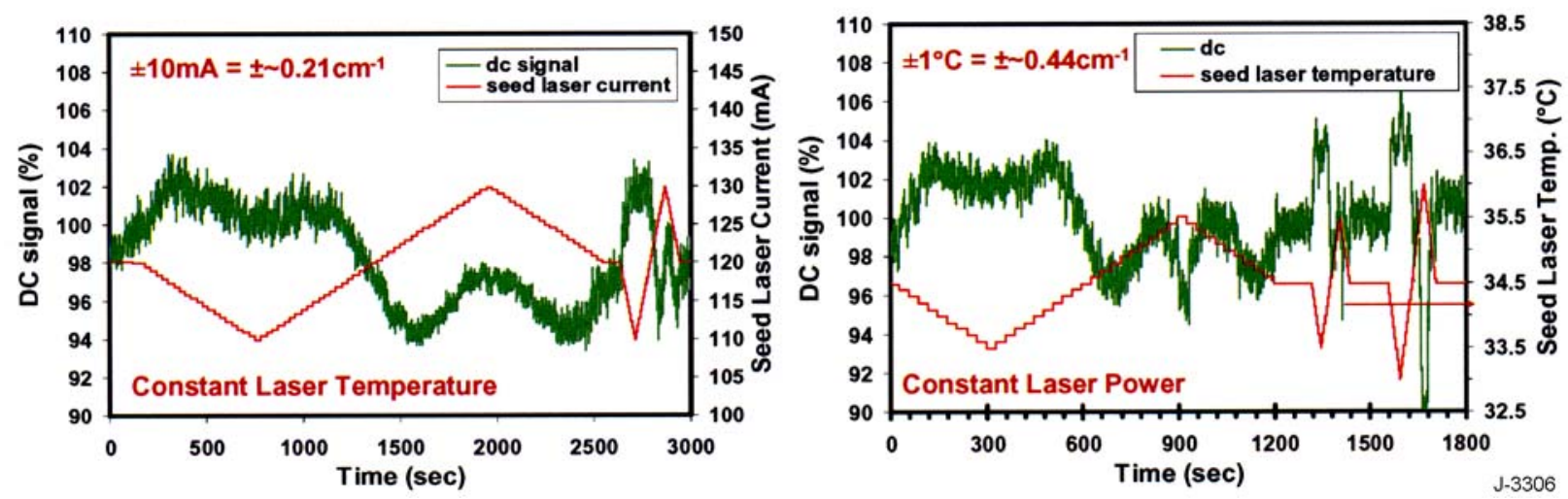

Figure 47. Output signal (power) from the EDFA versus wavelength via injection current (left) and temperature (right) laser tuning. The variable injection current experiment is held at a fixed temperature of $34.5^{\circ} \mathrm{C}$ and the variable temperature experiment is held at a fixed output seed laser power of $21 \mathrm{~mW}$.

\subsubsection{Task 6: Flight Test Preparation}

PSI completed plans for a flight test of the airborne RMLD and an initial Flight Test Plan was created in preparation for this event. The details are described in this document, which is attached to this report as Appendix D.

In short, the plan required a gas (or methane) leak rate of $5000 \mathrm{scfh}$ for a duration of about 2 hours. Initially, we had intended to simulate such a leak using several cylinders of methane to be released at an undefined location. Later, we made contact with the operator of a local high-pressure gas transmission pipeline and the operator agreed to participate in the tests by (safely) releasing gas at a nearby metering station. Plans were made to perform these tests during the week of October 11, 2006. These plans included the logistics of the tests, as well as details such as the design of a vibration isolating transceiver-mounting framework for interfacing with the selected aircraft. On October 3, the pipeline operator informed PSI that approval for the test was required from a third party, the owner of a LNG storage tank co-located with the metering station. For security reasons, the third party disapproved of flights over the LNG tank 
and forced cancellation of the planned tests. A rescheduling/relocation of the tests in the timeframe available was logistically not feasible.

In response, PSI secured a 2-month no-cost extension to the program and completed plans for a surrogate set of ground-based tests to simulate a low altitude flight test of the airborne RMLD system at speeds approaching those of a slow flying aircraft survey. In the time period between the cancelled flight test of early October and the creation of the Simulated Flight Test plan, a measurable amount of effort was spent in determining the feasibility of a flyover leak survey test of the sensor at PSI headquarters in Andover or an alternate facility that would still employ the survey aircraft as the sensor platform. Logistical and legal issues proved too great to overcome in the short timeframe, however, so the idea was abandoned for the alternate groundbased test plan.

In earlier ground-based tests, it was determined that the sensor system could operate with a detection range of at least a few hundred meters with the unamplified tunable diode laser as the interrogating laser. This is a sufficient stand-off range for low-level aerial survey, which typically must cruise at an altitude greater than $500 \mathrm{ft}$. As measurements could be made at this range unencumbered by the added noise from the laser amplifier, and very large open ranges are difficult to come by on the ground, the unamplified system at a range of a $\sim 500 \mathrm{ft}$ was embraced as the most optimal scenario for best sensor performance. The site for the earlier outdoor ground tests was unavailable at this time, so we searched for alternate sites that could provide several hundred feet of open space, were easily accessible, and not too trafficked. In the end, the simplicity of operating at the PSI facility was adopted. Though tree cover was a limiting factor, two relatively long open paths were identified between the PSI rooftop and locations in the business park roads. A road was required at the distal end, as the methane "leak" to be sensed was to be moved via automobile as described below. An aerial view of the two paths is shown in Figure 48.

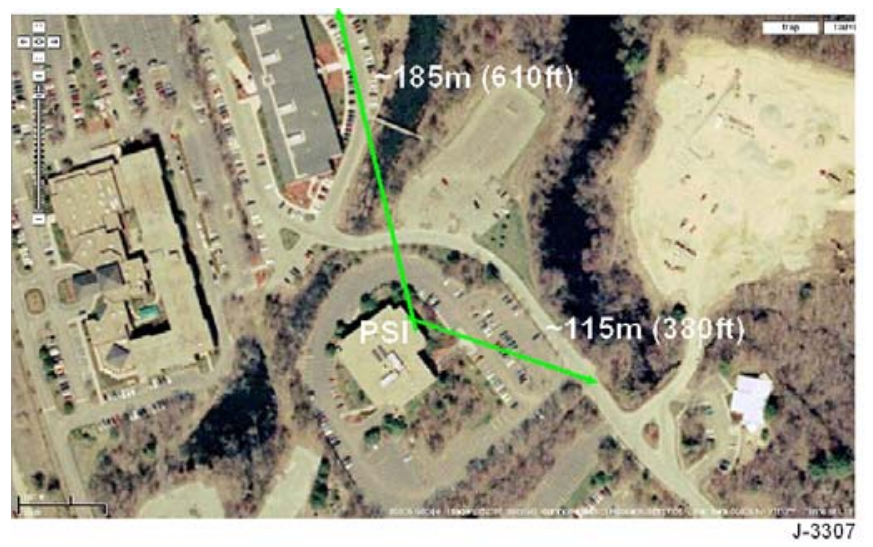

Figure 48. Aerial view of PSI facility and employed sensor-to-target paths. 
The shorter pathlength $(\sim 115 \mathrm{~m})$ ended up being the most utilized for these tests, as higher speeds of target motion could be obtained on this road. The longer path $(\sim 185 \mathrm{~m})$ was essentially a test for return signal strength at an aircraft-scale standoff distance. The views of these two paths from the transceiver (foreground) location atop the roof can be seen in Figure 49. Included in these images are the approximate locations and sizes of the telescope ocular field of view and the interrogating beam at the target. In the "fly by" measurements, the sensor transceiver remains steady, staring at these target locations while the sample "leak" is moved through these points.

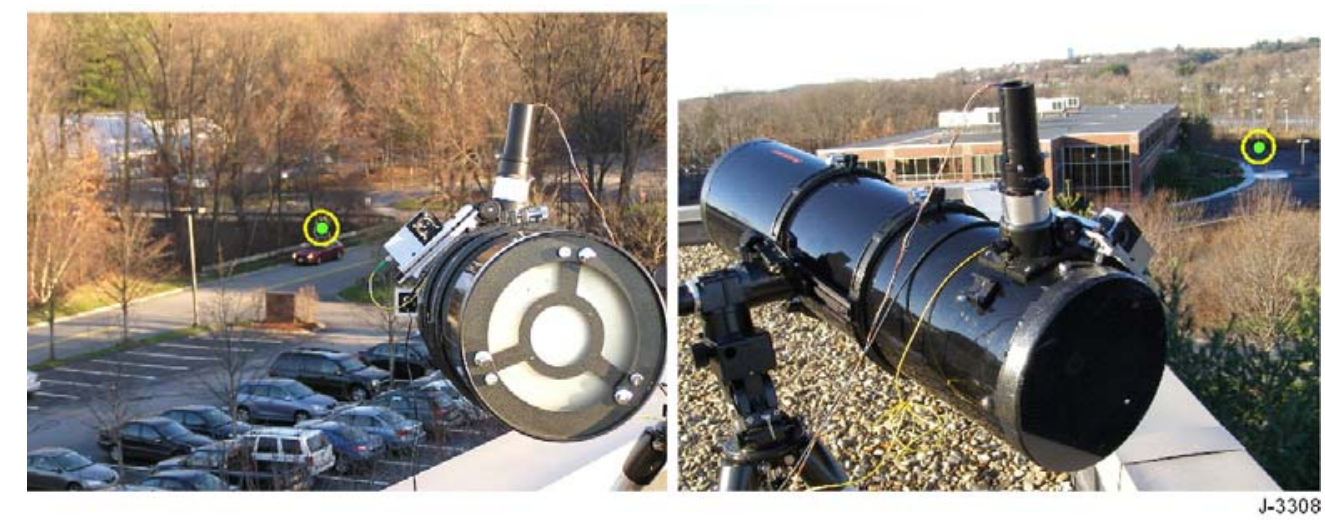

Figure 49. View from sensor transceiver to measurement location for the $115 \mathrm{~m}$ path (left) and $185 \mathrm{~m}$ path (right). Yellow circles indicate approximate telescope field of view and green circle indicates approximate laser footprint.

The sample "leak" is a transparent bag containing methane $\left(\mathrm{CH}_{4}\right)$ that is enclosed in a wooden frame with a plywood backing. This plywood provides the diffuse reflection received by the transceiver. The entire assembly, henceforth referred to as the "target", is $1.00 \mathrm{~m} \times 0.75 \mathrm{~m}$, with a frame aperture (or "leak" dimension) of $0.75 \mathrm{~m} \times 0.60 \mathrm{~m}$. The bag is filled with $10 \% \mathrm{CH}_{4}$ and balance $\mathrm{N}_{2}$ and has relatively uniform depth of $\sim 10 \mathrm{~cm}$. This yields a path-integrated concentration of $10,000 \mathrm{ppm}-\mathrm{m}$. Also, though, for the $115 \mathrm{~m}$ path, the target traverses the interrogation path at an attitude of approximately $30^{\circ}$, yielding an effective depth of $\sim 11.5 \mathrm{~cm}$, and thus a path-integrated concentration of 11,500ppm-m. To move the target through the sensor field of view at a relatively high speed (up to $50 \mathrm{mph}$ ), the target assembly is mounted to a rack assembly on the roof a car, as shown in Figure 50.

One modification of significance was made to the sensor as compared to the amplified high-altitude scenario. Specifically, an alternate tunable diode laser source at $1654 \mathrm{~nm}$ was employed in place of the $1618 \mathrm{~nm}$ laser. The $1618 \mathrm{~nm}$ laser was chosen for the relatively low absorption linestrength of methane at this wavelength that would keep the returned laser power from being strongly attenuated by the extremely long path of background methane $(\sim 30,000 \mathrm{ppm}-$ $\mathrm{m}$ at $15,000 \mathrm{~m}$ altitude). The anticipated performance of this high-altitude system was a noiseequivalent sensitivity of $\sim 100 \mathrm{ppm}-\mathrm{m}$ and was intended to pick out leaks at the 1000ppm-m scale. The absorption coefficient at the more typically employed $1654 \mathrm{~nm}$ absorption line is $\sim 100 \mathrm{x}$ stronger, yielding a noise-equivalent sensitivity of $\sim 1 \mathrm{ppm}-\mathrm{m}$ and a operational detection limit of $\sim 10 \mathrm{ppm}-\mathrm{m}$. This laser swap also required a switch of the bandpass interference filter in front of the detector in the transceiver to one centered at the $1654 \mathrm{~nm}$ line. 


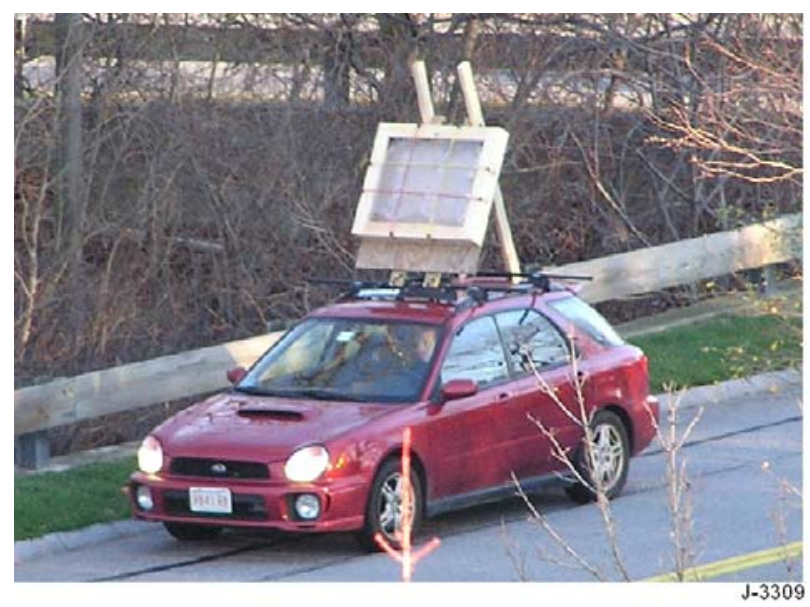

Figure 50. Methane target assembly mounted to the roof of an automobile.

The optics of the transceiver were otherwise unchanged from earlier outdoor testing. The optical axis of the telescope and the beam that is launched at the perimeter of the telescope are nearly parallel. They converge at a distance of $\sim 2000 \mathrm{~m}$, as the system was aligned on a remote water tower. The beam divergence was designed to be $\sim 3.33 \mathrm{mrad}$, but subsequent analysis suggests the divergence is closer to $5 \mathrm{mrad}$. This yields an approximate beam diameter of $0.58 \mathrm{~m}$ at the $115 \mathrm{~m}$ range and a diameter of $\sim 0.93 \mathrm{~m}$ at the $185 \mathrm{~m}$ range. The telescope ocular field of view is $\sim 3 \mathrm{x}$ larger at $\sim 15 \mathrm{mrad}$, but the detector field of view essentially overlaps the beam footprint. The camera field of view is $\sim 25 \mathrm{x}$ larger than the beam divergence at $0.122 \mathrm{rad}\left(7^{\circ}\right)$. This camera recorded images at $\sim 3 \mathrm{~Hz}$. Sample frames from recorded video data are shown in Figure 51. The methane sensor data was recorded at the usual $10 \mathrm{~Hz}$ rate. The intrinsic camera software and methane sensor software were combined into a common graphical interface for simultaneous viewing and file saving as shown in Figure 52. Graphed data include total received optical power (incl. background), 1f "carrier" signal, $2 \mathrm{f}$ "absorption" signal, and calculated pathintegrated methane concentration.
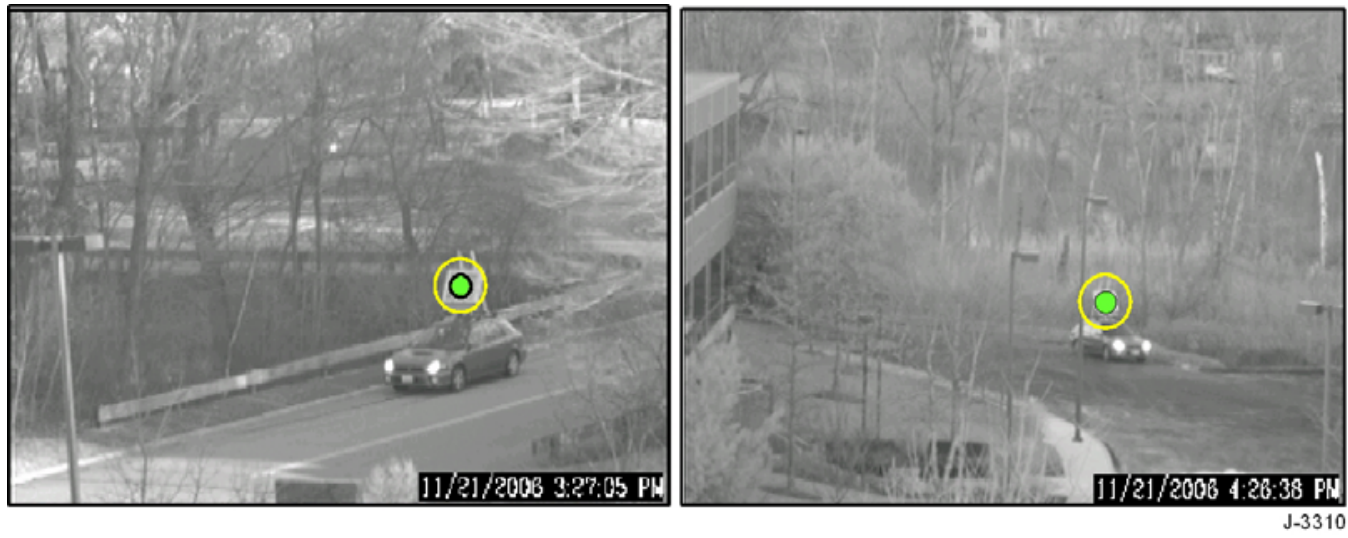

Figure 51. Recorded camera images from simulated flight test experiments at $115 \mathrm{~m}$ range (left) and $185 \mathrm{~m}$ range (right). Again, the telescope ocular field of view and laser footprint are indicated. 


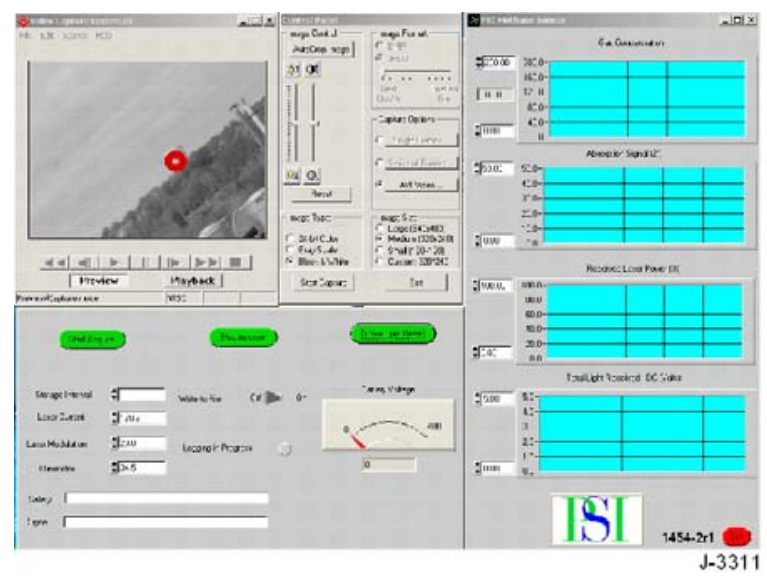

Figure 52. Graphical user interface employed in simulated flight test experiments. Red circle indicates sensor interrogation region. Sample image shown here is acquired during optical alignment on water tower.

\subsubsection{Task 7: Simulated Flight Test}

The first and majority of experiments were carried out on the $115 \mathrm{~m}$ standoff path. Again, the main reason for this was the ability to drive the target by at higher speeds at this location. Methane sensor data and video were recorded for 10 drivebys at speeds ranging from 10 to $50 \mathrm{mph}$. Three sample data records spanning this speed range are shown in Figure 53.
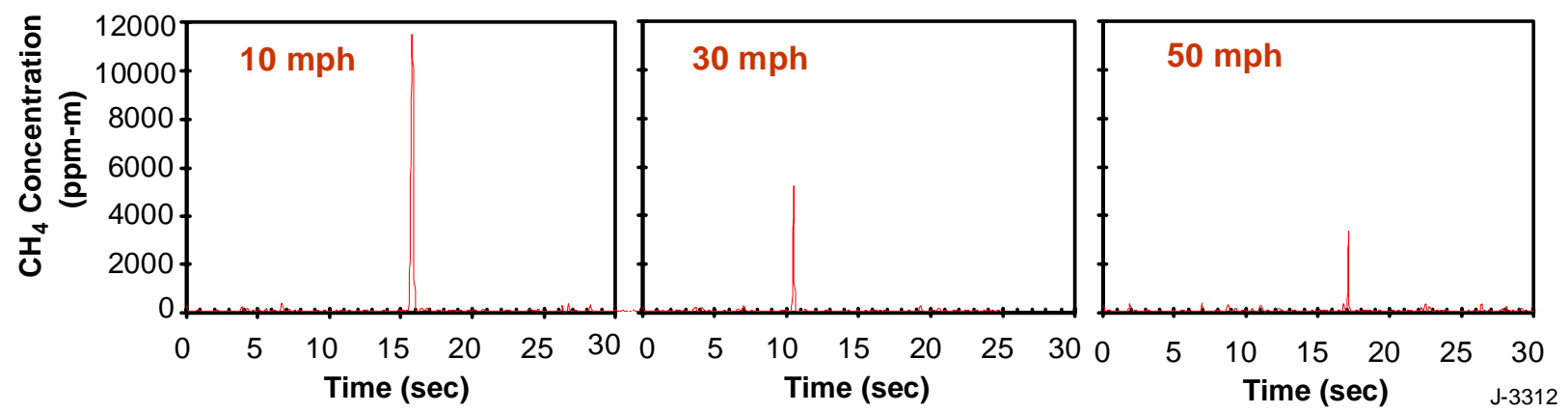

Figure 53. $10 \mathrm{~Hz}$ data records of $\mathrm{CH}_{4}$ concentration signals from the airborne remote methane leak detector (aRMLD) detecting a calibrated 11,500ppm-m gas sample passing through its field of view at $115 \mathrm{~m}$ range at three different speeds.

The effect that stands out immediately is that the signal level from a fixed "leak" strength of 11,500ppm-m decreases with increasing target velocity. At the slowest speed, the maximum expected signal is observed, but only for 1 of the $10 \mathrm{~Hz}$ data points. The reason for this is that at $10 \mathrm{mph}(\sim 4.5 \mathrm{~m} / \mathrm{s}), \mathrm{a} \sim 1 \mathrm{~m}$ target moves through a $\sim 1 \mathrm{~m}$ beam $(2 \mathrm{~m}$ traverse $)$ in $\sim 0.5 \mathrm{sec}$, yielding $\sim 5$ sample data points with some overlap between laser beam and the target and the middle data point with near perfect overlap yields the full 11,500ppm-m signal. An infinitely small beam would move across the target ( $1 \mathrm{~m}$ traverse) in $0.22 \mathrm{sec}$ yielding $\sim 2$ datapoints at full signal 
strength. When integrated, these two scenarios should yield the same answer. However, a running integrator averages in "zeros" at the maximum reading unless the integration time is matched to or smaller than the target/beam residence time.

At the other end of the spectrum, at $50 \mathrm{mph}(22 \mathrm{~m} / \mathrm{s}), \mathrm{a} \sim 1 \mathrm{~m}$ target moves into and out of the sensor's beam in $<0.1 \mathrm{sec}$, which is less than a full $0.1 \mathrm{sec}$ sample integration time. Any averaging of $0.1 \mathrm{sec}$ samples in this scenario reduces the contrast of "methane present" datapoints to "methane absent" datapoints. Also worth noting is that each $0.1 \mathrm{sec}$ sample is comprised of an average of 16 digital lock-in samples of $6.3 \mathrm{~ms}$ long digital records. The total output signal depends on how well the "overlap" start and end time match the signal integration start and end time. To complicate matters even a little further, though the target was made as large as feasibly possible, because the target and laser beam size are comparable, a slight misalignment of beam and target can have a noticeable effect. This alignment is more difficult to achieve while driving at the higher speeds. This scenario is, in fact, much more challenging than that likely to be observed at a real pipeline leak, where the leak cloud is likely to overfill the interrogating laser beam diameter. However, the results are useful to consider in terms of beam residence time in the leak cloud. Several factors influence this parameter in the aerial survey scenario: aircraft speed, aircraft altitude, beam divergence, and leak plume diameter. The results presented here can guide the selection of the controlled parameters just listed, assuming some educated estimate for a typical leak plume diameter.

Figure 54 presents a summary sensor detection limit, based on maximum "leak" signals during the target drive by at different speeds and rms noise levels on the signal when the target was not present. The detection limit reported is the noise-equivalent signal based on the signal/noise ratio (SNR) measured for the particular data set, calibrated against the known "leak" magnitude of $11,500 \mathrm{ppm}-\mathrm{m}$ (i.e. det. limit $=11,500 \mathrm{ppm}-\mathrm{m} / \mathrm{SNR}$ ). As can be seen, averaging only reduces the contrast, and therefore SNR, of the measurement. Without averaging, the detection limit levels out for slower survey speeds at $\sim 50 \mathrm{ppm}-\mathrm{m}$.

It is worth noting, also, that these particular data records were subject to a spurious noise contribution that may have been related to the power supply at the PSI rooftop. As can be seen in Figure 55, significant noise spikes are present in the data that are not generally seen in typical or previous unamplified aRMLD measurements. This is definitely not an optical signal contribution, as they occur with the sensor simply staring at a passive background, without even any reflective surfaces in the field of view, as might be found on the automobile. Presuming that this effect can be alleviated, detection limits would drop measurably. Figure 56 illustrates the maximum signal levels for the various data records, as well as the calculated rms noise levels, with and without inclusion of the noise spikes. Recalculated detection limits based on the optimal noise limit are shown in Figure 57. Without averaging, the detection limit levels out for slower survey speeds at $\sim 7 p p m-m$. 


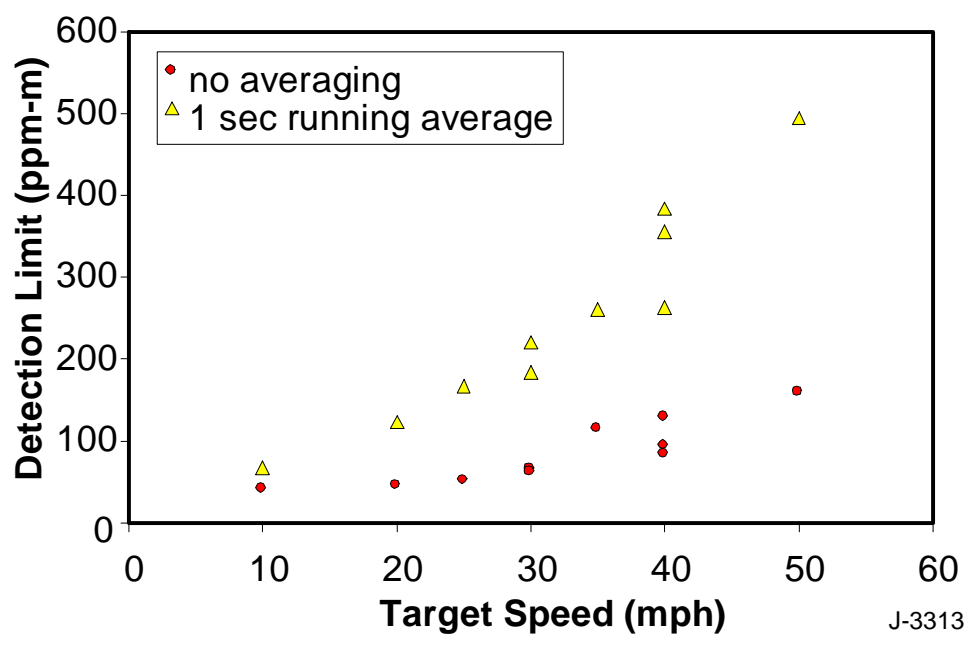

Figure 54. Calculated detection limits for the airborne remote methane leak detector (aRMLD) versus target speed, based on the SNR of data records such as in Figure 53. Red circles represent values calculated from the raw data, and the yellow circles represent values calculated with a $1 \mathrm{sec}$ running average applied to the data.

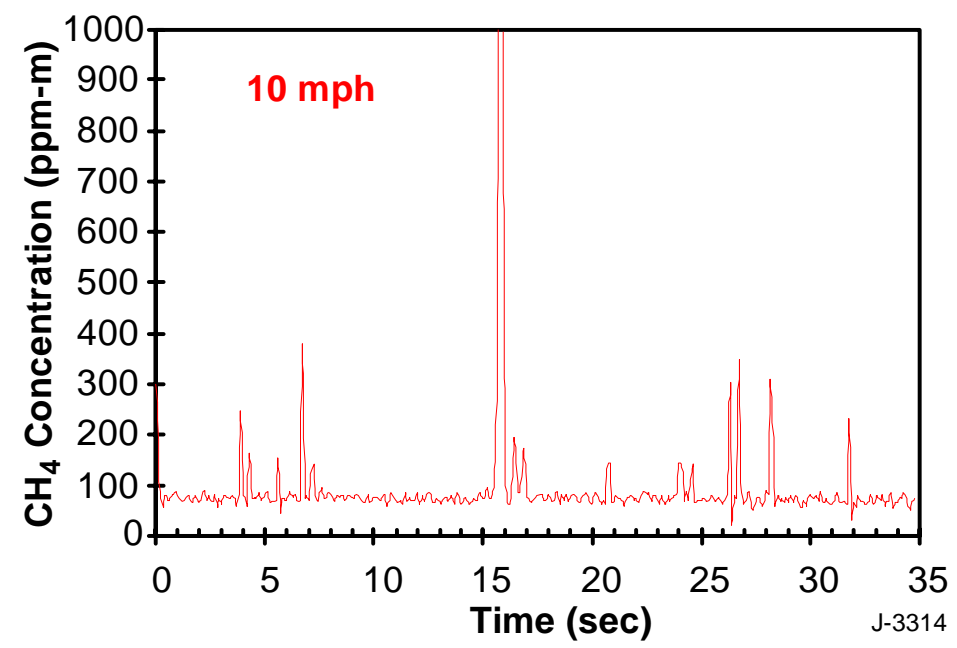

Figure 55. Zoom of $10 \mathrm{~Hz}$ data record of $\mathrm{CH}_{4}$ concentration signals from the airborne remote methane leak detector (aRMLD) detecting a calibrated 11,500ppm-m gas sample passing at $10 \mathrm{mph}$. 

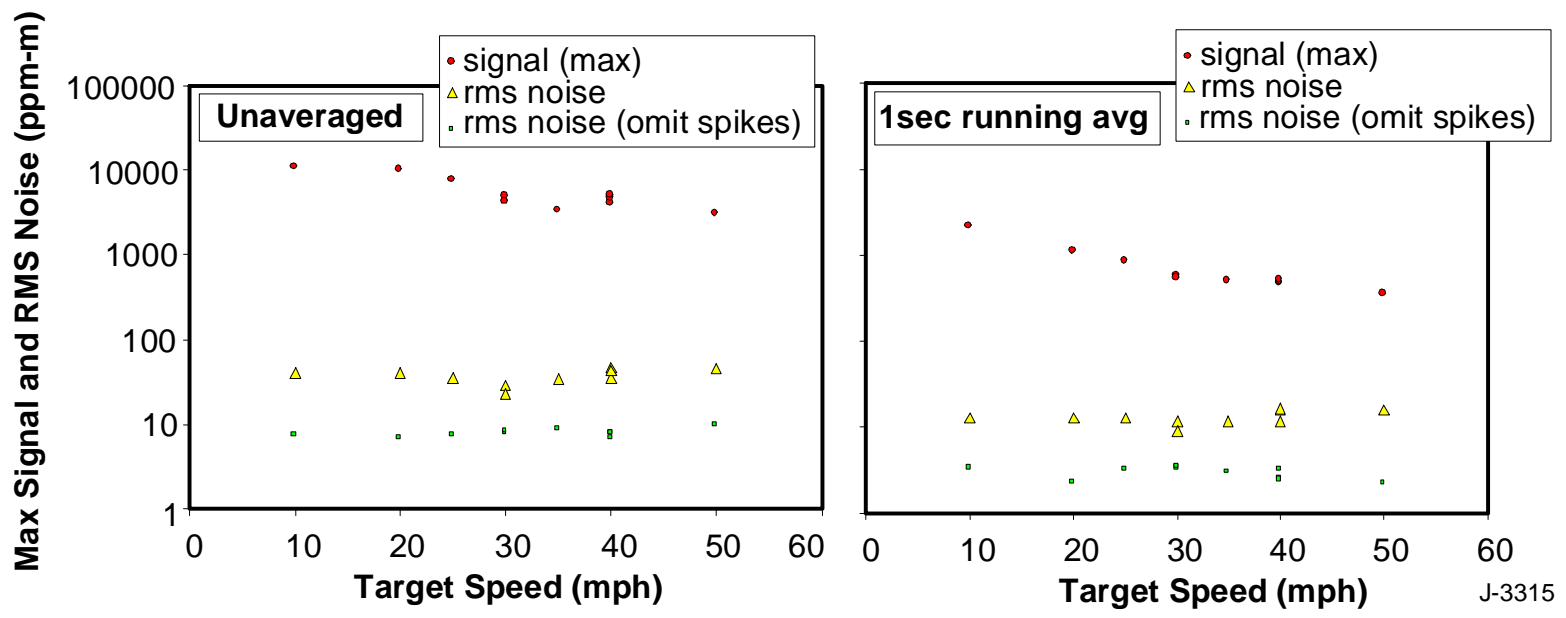

Figure 56. Recorded maximum (red) and rms noise signals (with (yellow) and without (green) noise spikes included) for the airborne remote methane leak detector (aRMLD) versus target speed.

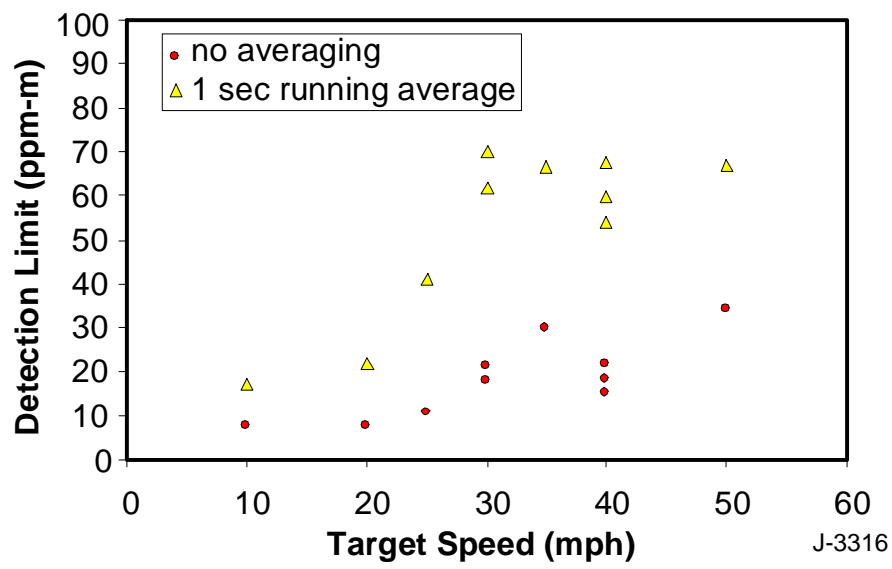

Figure 57. Calculated detection limits for the airborne remote methane leak detector (aRMLD) versus target speed, based on the SNR of data records such as in Figure 7, with noise spikes removed. Red circles represent values calculated from the raw data, and yellow triangles are calculations with a $1 \mathrm{sec}$ running average applied to the data.

Several data records were also recorded at the $185 \mathrm{~m}$ range (see Figures 48, 49, and 51). This geometry provided a target that was more normal to the sensor beam, but the smaller road space necessitated slower speeds, the longer range yielded a more challenging beam/target alignment, and the expected signal reduction from underfilling the sensor beam with the sample was evident in the data. Figure 58 illustrates signals acquired from a parked target with a stable sensor and with a scanning sensor and show that the beam is in fact $\sim 46 \%$ underfilled $(0.93 \mathrm{~m}$ beam \& $0.75 \mathrm{~m} \times 0.60 \mathrm{~m}$ sample), as the $10,000 \mathrm{ppm}-\mathrm{m}$ sample yields only a $4600 \mathrm{ppm}-\mathrm{m}$ signal. Figure 59 illustrates the signals generated with the target driven through the sensor field of view. The somewhat smaller signals here illustrate the challenge of driving the target through the precise optimum beam location. 


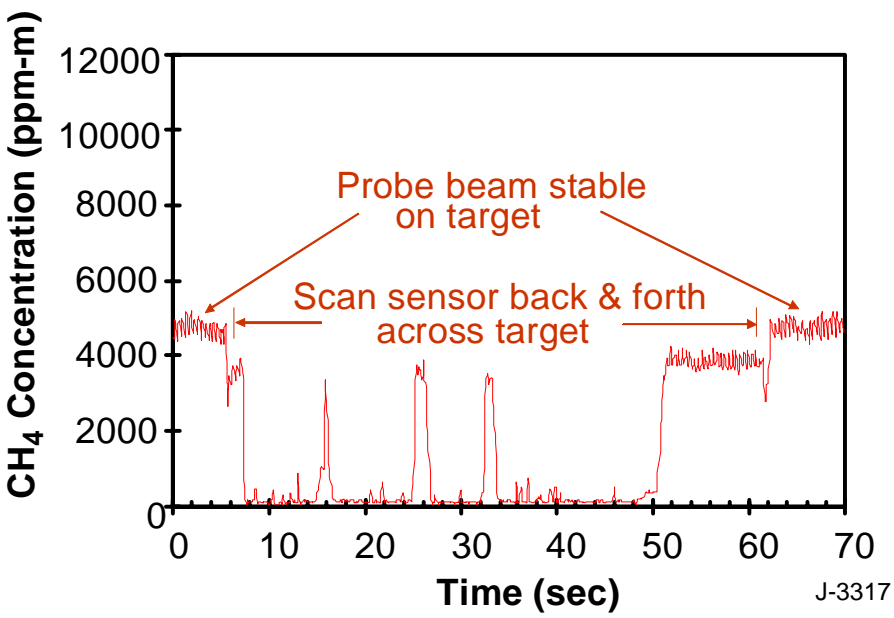

Figure 58. $10 \mathrm{~Hz}$ data records of $\mathrm{CH}_{4}$ concentration signals from the airborne remote methane leak detector (aRMLD) detecting a calibrated 10,000ppm-m gas sample at a $185 \mathrm{~m}$ range. From the 2 to $50 \mathrm{sec}$ mark, the sensor is panning across the target at what would equivalently be an unsteady target movement speed of $\sim 5-10 \mathrm{mph}$.

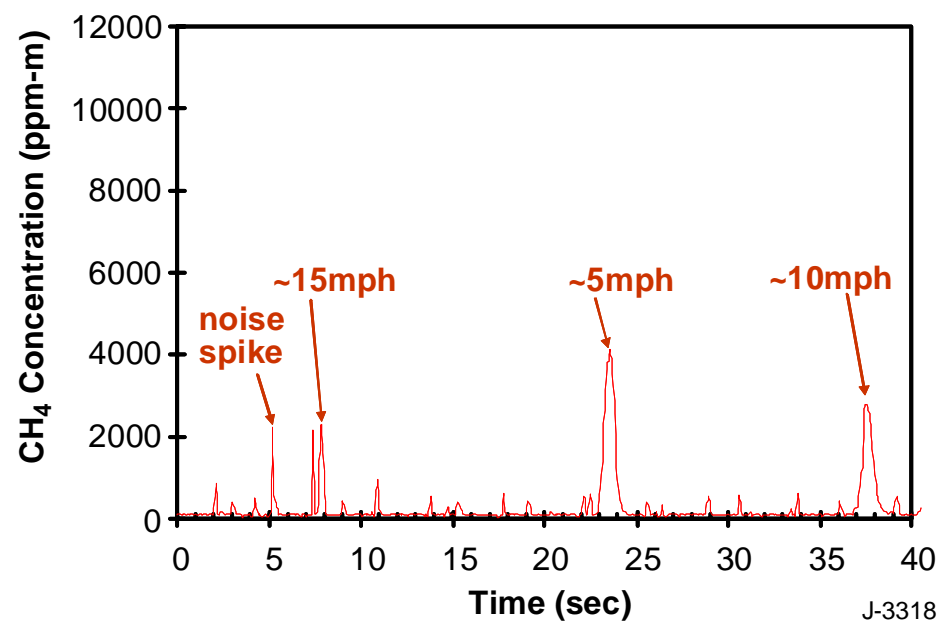

Figure 59. $10 \mathrm{~Hz}$ data records of $\mathrm{CH}_{4}$ concentration signals from the airborne remote methane leak detector (aRMLD) detecting a calibrated 10,000 ppm-m gas sample passing through its field of view at a $185 \mathrm{~m}$ range at three different speeds. First \& last passes are with target moving forward, middle pass has target moving backward.

The absolute magnitude of the received laser power at the $185 \mathrm{~m}$ range was $\sim 0.7 \mathrm{nW}$, about $40 \%$ that received from the $115 \mathrm{~m}$ range $(\sim 1.6 \mathrm{nW})$. The carrier $(1 \mathrm{f})$ signal for the $185 \mathrm{~m}$ range has a SNR of $\sim 25$ (though this is often "common mode" with the absorption (2f) signal and is thus cancelled to a varying extent when ratioed with $2 \mathrm{f}$ ), so we are approaching the range limit of the system. The "zero" signal rms noise magnitude of the path-integrated $\mathrm{CH}_{4}$ concentration calculation is up by a factor of 2 at this range, compared to the $115 \mathrm{~m}$ range, 
suggesting that the SNR of the carrier signal is affecting the output. Another factor of four in range ( 750m) might be possible, reducing the received power by $16 \mathrm{x}$ and the SNR of the carrier signal to $\sim 6$, and the SNR of the $\mathrm{CH}_{4}$ signal by another $4 \mathrm{x}$ as an estimate (from the $185 \mathrm{~m} / 115 \mathrm{~m}$ factor of $2 \mathrm{x}$ ). Assuming a sufficiently large beam/target temporal and spatial overlap (yielding full analyte signal in at least one $0.1 \mathrm{sec}$ reading), this would push the SNR of the system (w/o noise spikes) into the $\sim 200$ range for the 10,000ppm-m sample and the detection limit therefore to $\sim 50 \mathrm{ppm}-\mathrm{m}$. 


\section{CONCLUSIONS}

\section{Summary of Significant Accomplishments}

- PSI has designed and assembled a prototype airborne remote methane leak detector (aRMLD) sensor suitable for insertion and testing in a survey-capable aircraft.

- PSI has completed a study of the performance characteristics of the simultaneously enabling and limiting component of the sensor system, an erbium-doped fiber amplifier (EDFA) and its impact on the wavelength modulation spectroscopy (WMS) detection technique. While the EDFA performance is not as good as we had expected prior to this program, the knowledge gained from this work is very valuable for identifying characteristics that will enable future performance improvements.

- PSI has performed an extensive set of ground-based measurements with the prototype system, both amplified and unamplified for characterization purposes and also in a simulation of a low-altitude leak survey scenario.

- PSI and our cost share partner Heath Consultants have received significant interest in a lowaltitude version of the airborne RMLD, one that surveys transmission pipelines from a helicopter flying at altitudes of $\sim 500 \mathrm{ft}$ AGL. This can be accomplished, as demonstrated, without the EDFA, but coupled with the large transceiver developed for the high-altitude RMLD. PSI and Heath are discussing means for addressing this opportunity. 


\section{REFERENCES}

1. Allen, M.G., Carleton, K.L., Davis, S.J., Kessler, W.J., Otis, C.E., Palombo, D., and Sonnenfroh, D.M., "Ultra-Sensitive Dual-Beam Absorption and Gain Spectroscopy: Applications for Near-IR and Visible Diode Laser Sensors," Applied Optics 34(18), p. 3240-3249, 1995.

2. Allen, M.G. and Kessler, W.J., "Simultaneous Water Vapor Concentration and Temperature Measurements Using 1.31 $\mu \mathrm{m}$ Diode Lasers," AIAA J. 34(3), pp. 483-488, 1996.

3. Allen, M.G., "Diode Laser Absorption Sensing of Gas Dynamic and Combustion Flows," an invited review for Measurement Science and Technology 9(4), pp. 545-562, 1998.

4. Wainner, R.T., Green, B.D., Allen, M.G., White, M.A., Stafford-Evans, J., and Naper, R., "Handheld, batter-powered near-IR TDL sensor for stand-off detection of gas and vapor plumes," Appl. Phys. B, 75(2-3), pp. 249-254, 2002.

5. see IPG Photonics web-site www.ipgphotonics.com, ELT Series EDFA and ELR series fiber laser product pages.

6. IPG Photonics Newsletter, “The Active Fiber,” Issue No. 1, January 2004. 
PSI-1454

\title{
Appendix A
}

\section{Research Management Plan}

\section{High-Altitude, Aerial Natural Gas Leak Detection System}

\author{
P.O. Number: DE-FC26-04NT42268 \\ Prepared by: \\ Mickey B. Frish \\ Physical Sciences Inc. \\ 20 New England Business Center \\ Andover, MA 01810-1077 \\ Prepared for:
DOE - National Energy Technology Laboratory
626 Cochrans Mill Road
P.O. Box 10940
Pittsburgh, PA 15236-0940

November 2004

\section{ACKNOWLEDGEMENT STATEMENT:}

This report was prepared with the support of the U.S. Department of Energy, under Award No. DE-FC26-04NT42268. However, any opinions, findings, conclusions, or recommendations expressed herein are those of the author(s) and do not necessarily reflect the view of the DOE.

DISCLAIMER

This report was prepared as an account of work sponsored by an agency of the United States Government. Neither the United States Government nor any agency thereof, nor any of their employees, makes any warranty, express or implied, or assumes any legal liability or responsibility for the accuracy, completeness, or usefulness of any information, apparatus, product, or process disclosed, or represents that its use would not infringe privately owned rights. Reference herein to any specific commercial product, process, or service by trade name, trademark, manufacturer, or otherwise does not necessarily constitute or imply its endorsement, recommendation, or favoring by the United States Government or any agency thereof. The views and opinions of authors expressed herein do not necessarily state or reflect those of the United States Government or any agency thereof. 
This document is the Research Management Plan comprising Task 1 of this project. Figure 1 shows the Work Breakdown Structure (WBS), schedule for each Task and major subtasks, and approximate planned expenditures for each Task, broken down by sub-task where appropriate. The WBS follows the Statement of Work (SoW). The narrative text below summarizes the tasks and supplements the SoW where needed for clarity. Major Tasks and Summary subtasks are Boldfaced black timelines in Figure 1. Individual subtasks are shown as blue timelines. Milestones are shown as diamonds. Planned expenditures funded by DoE are shown in black type. Expenditures that PSI plans to contribute as cost sharing are shown in red. The expected spending plan is shown graphically in Figure 2.

\section{Project Objective}

The objective of this project is to develop and demonstrate cost-effective and powerefficient advanced remote sensing technology able to detect and quantify, from an aerial vehicle, natural gas leaking from a high-pressure pipeline. This leak detection technology will ultimately be capable of operating at heights of 50,000 ft and higher.

\section{Scope of Work}

Physical Sciences Inc. (PSI) will utilize and extend the tunable diode laser absorption spectroscopy (TDLAS) technology embedded within the handheld, battery-powered laser-based Remote Methane Leak Detector (RMLD) product, developed by PSI, to build and demonstrate a system for standoff sensing, from high altitudes, of natural gas distribution/transmission pipeline leaks. The solid-state, near-IR lasers within RMLD will be enhanced with scalable, high-power optical fiber amplifiers to provide a compact, power-efficient sensor to be flown upon an aerial platform having an operational ceiling of 10,000 ft. PSI will design, assemble and flight test an Experimental Prototype (EP) sensor system intended to demonstrate the potential to further scale the device to achieve leak detection from altitudes in excess of 50,000 ft.

\section{Major Tasks}

\section{Task 1 -- Research Management Plan}

\section{Objective}

Establish a plan that guides the program work, schedule, and budget, facilitates communication among team members, and enables progress to be tracked.

\section{$\underline{\text { Work }}$}

PSI will develop a work breakdown structure (WBS) and supporting narrative that concisely addresses the overall project. The narrative will summarize the technical objectives and technical approach for each Task and, where appropriate, for each subtask. The plan includes schedules and expected expenditures for each Task, plus all major milestones and decision points. This report will be submitted to the DOE Contracting Officer's Technical Representative (COR) for review and comment, and be subsequently finalized incorporating any DoE input. 


\section{Task 2 -- Technology Status Assessment}

\section{Objective}

Review and communicate the state-of-the-art of the technology to be developed in this program to clearly communicate the advancements that the research is intended to achieve.

$\underline{\text { Work }}$

PSI will review the state-of-the-art of airborne surveying for natural gas pipeline leaks and submit a Technology Status Assessment summary report. The short report will include both positive and negative aspects of each existing technology. This report will be submitted to the DOE Contracting Officer's Technical Representative (COR) for review and comment, and be subsequently finalized incorporating any DoE input.

\section{Task 3 -- System Architecture and Specification}

\section{Objective}

Create a written document describing, in the form of a set of specifications, the technology to be developed meeting the program goals. These specification document will guide the design of the technology system, serve as a basis for ongoing discussions about the technology, and may be modified or refined as the technology development progresses.

$\underline{\text { Work }}$

PSI will develop and document a complete set of initial specifications describing the technology under development. To complete this Task, PSI will identify the major system components and the aircraft to be used for flight tests in Task 7. The major components will be selected to be compatible with aircraft size, weight, and power requirements. Based on these components, PSI will determine the expected detection range, sensitivity, footprint at ground, and operational speed. This information will be documented in a Preliminary Specification.

A draft of the specification document will be submitted to the DOE Contracting Officer's Technical Representative (COR) for review and comment. The Preliminary Specification will subsequently be modified incorporating any DoE input.

\section{Task 4 -- System Design, Fabrication, and Assembly}

\section{Objective} Task 7.

Design and build the Experimental Prototype (EP) leak sensor that will be flight tested in

$\underline{\text { Work }}$ 
This Task comprises six Summary Subtasks intended to result in a robust a verified device suitable for field testing.

\subsection{Benchtop Sensor Assembly and Preliminary Laboratory Testing}

Long lead components (e.g. the fiber amplifier) will be purchased early in the program. Other major system components will be purchased on a schedule permitting nearly concurrent delivery of all major components. The major components will be assembled in a benchtop laboratory configuration and tested to verify functionality and to learn how they work collectively.

\subsection{Preliminary Airborne Package Design}

Based on the specification and selection of major components, PSI will complete a preliminary design of the airborne sensor configuration. The preliminary design will include top level assembly drawings showing how the components will fit into the airborne platform.

\subsection{Preliminary Design Review}

The preliminary package design will be presented to an audience of experts for review and critique. The design will be modified as appropriate in response to the review.

\subsection{Detailed Design of the Engineering Prototype}

Based on the Preliminary Design, details of the design for the system to be flight tested will be completed. This will result in a Bill of Materials for the system specifying all purchased and manufactured components and drawings specifying their assembly configuration.

\subsection{Design Review}

Based on knowledge gained in subtask 4.1, the design completed in subtask 4.4 will be modified and then presented to a panel of experts for review and critique. The design will again be modified as appropriate in response to the review.

\subsection{Assemble Engineering Prototype}

PSI will assemble the sensor according to the design specified upon completion of subtask 4.5

\section{Task 5 -- Laboratory/Outdoor Testing}

\section{Objective}

Test and optimize the performance the EP system built in Task 4. Evaluate readiness for flight testing. 
$\underline{\text { Work }}$

PSI will test the EP system from the PSI rooftop, safely transmitting the laser beam to distant topographic targets of convenience. The system will be calibrated and optimized to evaluate and optimize optical power output, methane sensitivity, interferences, and collection efficiency. Detection algorithms will also be optimized to accommodate rapidly changing backgrounds. These detection algorithms will be based upon those being developed concurrent with this program to improve ability of the handheld RMLD to discriminate between leaks and ambient methane in situations of changing topography. The improved algorithms will be provided to this program as part of the PSI cost sharing contribution.

After optimizing the system, PSI will compare its performance against the system specifications (as defined in Task 3) and refine system and specifications as required. PSI will compile and evaluate the data and determine whether the system as built meets the requirements for airborne testing. The data and conclusions will be presented to the DOE Contracting Officer's Technical Representative (COR) for review and comment. Upon completion of the review, a decision to proceed with flight testing or not will be taken. This milestone will complete the first year of the program.

\section{Task 6 -- Preparation for Flight Demonstration and Installation}

\section{Objective}

Prepare sensor system for flight testing.

$\underline{\text { Work }}$

PSI will prepare the Engineering Prototype system for flight testing. This includes coordinating with the aircraft operator to specify details such as power connections, installation supports, safety considerations and flight test protocols. PSI and the aircraft operator will define a limited set of shock and vibration tests that the sensor system must survive prior to flight to assure flight safety. PSI will contract a specialty service to perform these tests. Upon completion of the safety tests and any re-configuration of the sensor to accommodate aircraft installation, a final test on the PSI rooftop will be completed to assure and optimize sensor performance.

\section{Task 7 -- Flight Test Preparation, Execution, Analysis, and Altitude Scaling}

\section{$\underline{\text { Objective }}$}

Prepare test protocols, test sites, and conduct flight tests. Analyze results and scale to higher altitudes.

$\underline{\text { Work }}$ 
PSI will contract with the aircraft provider to conduct two flight tests series. PSI and the aircraft operator, in consultation with the DOE Contracting Officer's Technical Representative, will select test sites and define a test protocol. PSI will prepare the test site, the aircraft operator will prepare the aircraft for sensor installation, the sensor will be installed, and flights conducted in accordance with the protocol.

In each test series, the aircraft will be flown at least three different altitudes as PSI personnel collect data on several methane clouds created by simulated gas leaks. Collected data will include measurements of return signal levels, providing information needed to guide future system design. Supporting information on solar illumination and wind conditions will also be collected.

PSI will analyze the data from these observations to determine sensitivity, altitude scaling and compare EP actual performance against its specification goals. 


\section{List of Milestones}

1. System Specification complete

2. Benchtop sensor system activated

3. System Design completed and reviewed

4. Ground-based outdoor testing completed

5. Flight test readiness completed

6. Flight tests completed

7. Data analysis and altitude scaling completed
15 January 2005

30 April 2005

30 June 2005

30 September 2005

15 May 2006

15 August 2006

15 September 2006 


\section{List of Deliverables}

1. Research Management Plan

1 November 2005

2. Technology Assessment Report

1 December 2004

3. System Specification

15 January 2005

4. Financial Status Reports

Quarterly

5. Biennial Progress Reports

Bi-annually

6. Final Technical Report

30 September 2006 


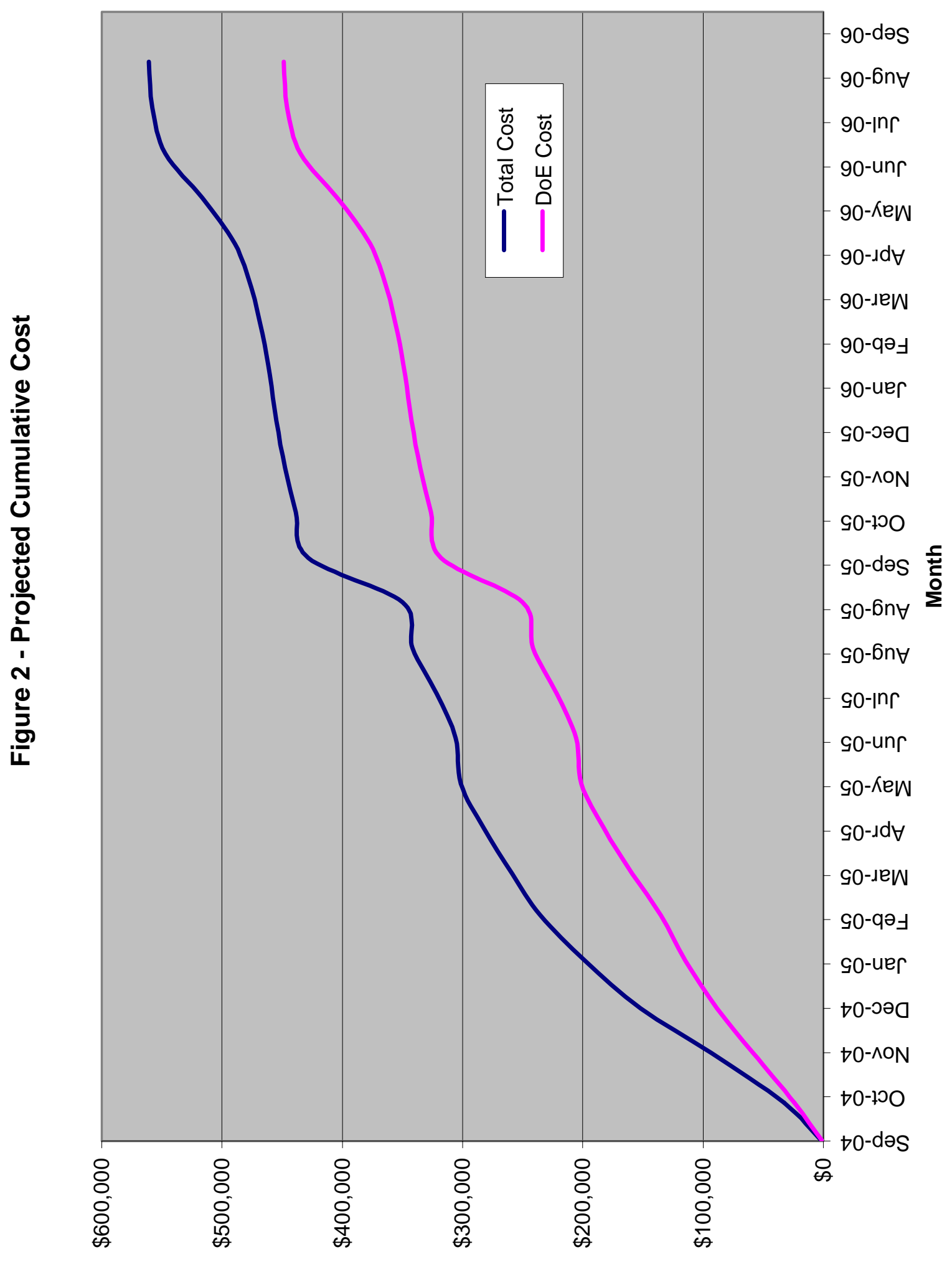




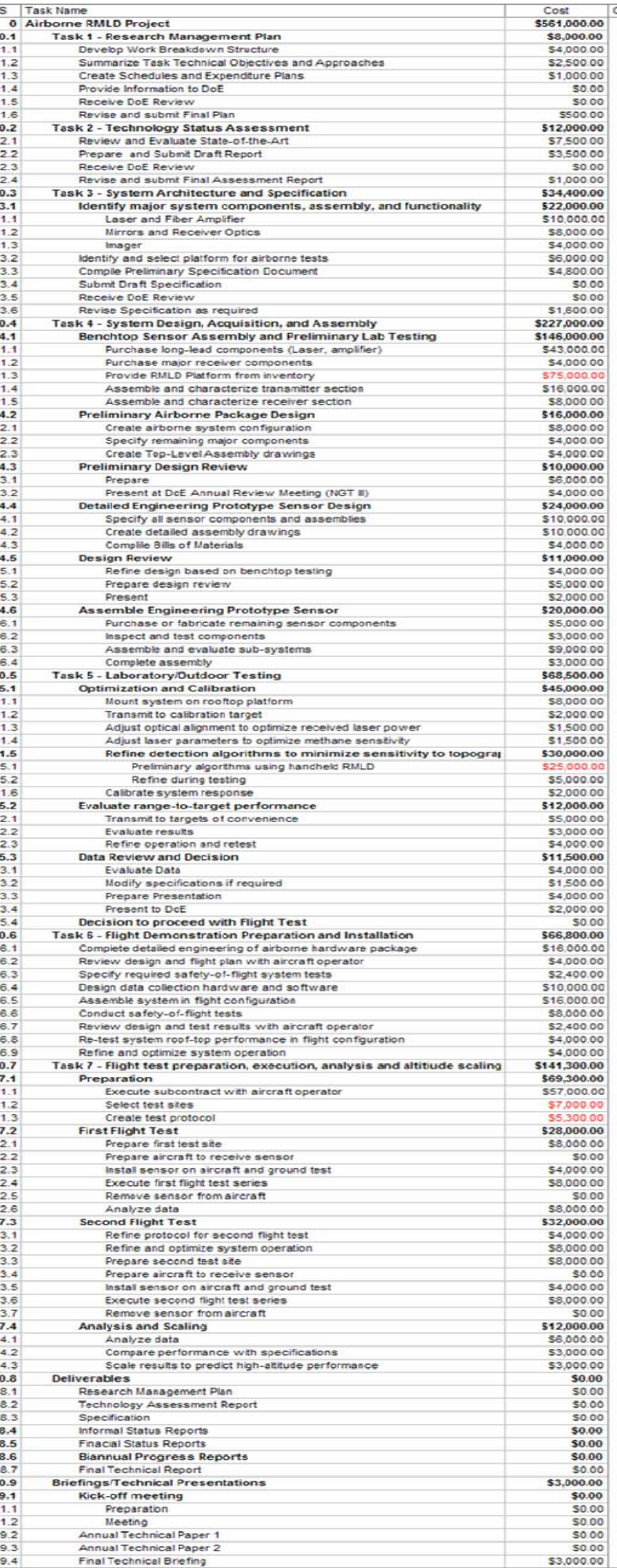

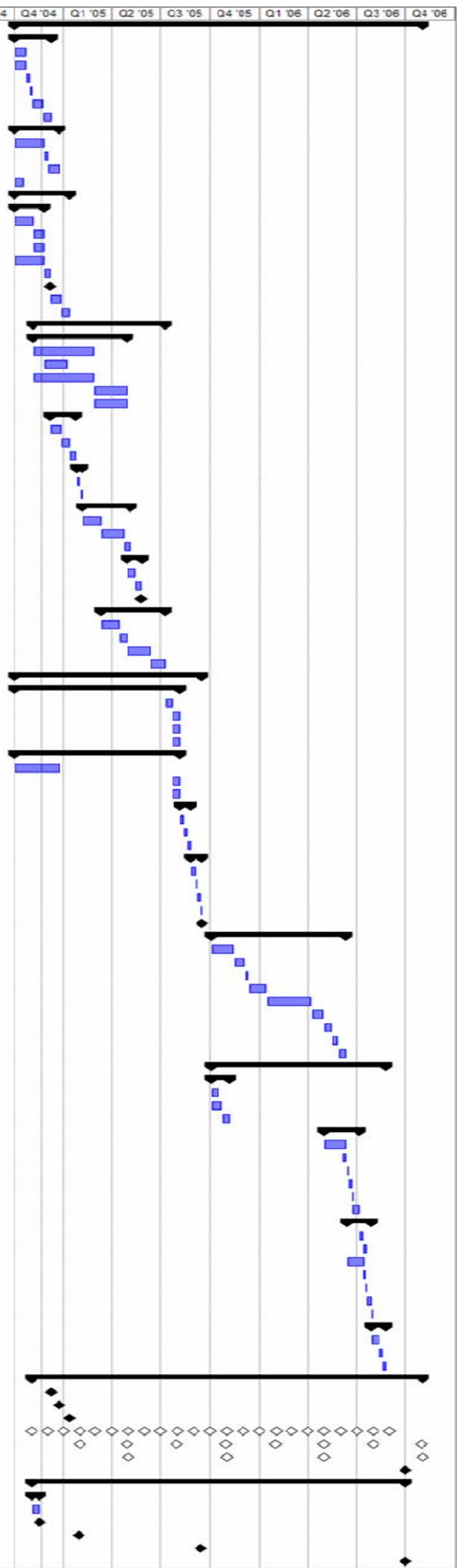

Figure 1. Work Breakdown Structure 
PSI-1454

\title{
Appendix B
}

\section{Technology Status Assessment}

\section{High-Altitude, Aerial Natural Gas Leak Detection System}

\author{
Contract No. DE-FC26-04NT42268
}

\author{
Prepared by: \\ Mickey B. Frish \\ Physical Sciences Inc. \\ 20 New England Business Center \\ Andover, MA 01810-1077 \\ Prepared for: \\ National Energy Technology Laboratory \\ U.S. Department of Energy \\ 3610 Collins Ferry Road, P.O. Box 880 \\ Morgantown, WV 26507-0880
}

December 2004

ACKNOWLEDGEMENT STATEMENT:

This Technology Status Assessment was prepared with the support of the U.S. Department of Energy, under Award No. DE-FC26-04NT42268. However, any opinions, findings, conclusions, or recommendations expressed herein are those of the author(s) and do not necessarily reflect the view of the DOE.

DISCLAIMER

This report was prepared as an account of work sponsored by an agency of the United States Government. Neither the United States Government nor any agency thereof, nor any of their employees, makes any warranty, express or implied, or assumes any legal liability or responsibility for the accuracy, completeness, or usefulness of any information, apparatus, product, or process disclosed, or represents that its use would not infringe privately owned rights. Reference herein to any specific commercial product, process, or service by trade name, trademark, manufacturer, or otherwise does not necessarily constitute or imply its endorsement, recommendation, or favoring by the United States Government or any agency thereof. The views and opinions of authors expressed herein do not necessarily state or reflect those of the United States Government or any agency thereof. 


\section{Introduction}

With support by the Department of Energy, Physical Sciences Inc. (PSI) has initiated a project to develop and demonstrate a laser-based sensor able to rapidly and autonomously, from a high-altitude platform, survey for leaks from natural gas transmission pipelines over large and remote areas. The ultimate goal of the technology is to enable surveys from unmanned aerial vehicles (UAVs) operating as high as $80,000 \mathrm{ft}$. The sensor is an enhancement of the Remote Methane Leak Detector (RMLD) developed recently by PSI. The RMLD is an example of a standoff sensor using the technique of Tunable Diode Laser Absorption Spectroscopy (TDLAS). Laser light emitted from a transceiver illuminates a passive surface (i.e. the ground). Using the well-understood principles of Wavelength Modulation Spectroscopy (WMS), the RMLD signal processor analyzes the signal embedded in the small amount of laser light reflected back to the transceiver and quantifies the amount of gas along the laser path.

The purpose of this Technology Assessment is to evaluate the state-of-the-art in laserbased and aerial natural gas leak detection, and understand how the enhanced RMLD technology to be developed provides a significant advancement.

\section{Background}

The US natural gas transmission system comprises approximately 250,000 miles of pipeline, 1700 transmission stations and 17,000 compressors. Maintaining the security and integrity of this system is a continual process of searching for, locating, and repairing leaks. Performing leak surveys is very labor intensive, in part because all currently used leak survey tools, including the traditional Combustible Gas Indicators (CGI) and Flame Ionization Detectors (FID), as well as the relatively recent Optical Methane Detection (OMD), must be physically immersed within a leak plume to detect it. CGI and FID tools both draw gas into a combustion chamber and analyze the products of combustion to quantify local gas concentrations. The OMD projects an infrared beam across a short $(\sim 1 \mathrm{~m})$ optical path open to the ambient air ("short path"), and determines by spectroscopy the concentration of gas within the optical path. The short optical path must encompass the gas to detect it. Short-path TDLAS, configured optically like an OMD but with better sensitivity, has been utilized for leak detection.

These techniques can and have been used for airborne leak surveying. To perform these surveys, a light airplane or helicopter flies no higher than a few hundred feet above the pipeline. Significant leaks create a plume that is intercepted by the aircraft and detected by the on-board instrumentation.

The RMLD is a novel configuration of the highly sensitive and selective TDLAS. It projects onto a distant surface the infrared beam emitted by a telecommunications-style diode laser. An optical fiber cable connects the laser to the transceiver which transmits the beam and receives scattered laser light. It senses the path-integrated concentration of methane between the transceiver and the illuminated surface. Because the RMLD is intended for use in walking leak surveys, it was designed to be handheld, lightweight, and power efficient. To accommodate these attributes, the sensor has a maximum range of about 100 feet, making it unsuitable for leak surveying from an aircraft. The current project will enhance the RMLD technology capability, 
extending its range to several thousand feet in a fashion that can be further extended to tens of thousands of feet, thereby enabling rapid airborne survey of large areas. To modify the RMLD for use in the airborne platform, three aspects of it will be modified: 1) the transmitted laser power will be increased by use of an optical fiber amplifier; 2) the size of the optical receiver will be increased; and 3) the laser wavelength will be changed.

\section{Alternative Leak Sensing Technologies}

To understand the advantage offered by the amplified RMLD, we compare below the attributes of various technologies available for leak surveying. The attributes of interest include: 1) the capacity for remote detection, meaning that the sensor need not be in close contact with the leak plume; 2) capability for survey from a moving platform, be it a surface vehicle or an aircraft; and 3) suitability for airborne surveying. If suitable for flight, a maximum altitude is given, if known.

\section{Combustible Gas Indicators (CGI)}

Principle of Operation: Catalytic Combustion of Gas Sample Sampling Method: Extractive (response time few seconds)

Sensitivity: Typically measures percentage gas concentration Advantages: Characterizes high concentrations of gas

Disadvantages: Does not detect low concentrations; sampling probe must be embedded within gas plume. Senses all combustible gases, not only natural gas.

Suitable for remote leak surveying: No

Suitable for mobile leak surveying: No - too slow and insensitive

Suitable for airborne leak surveying: No.

\section{Flame Ionization Detectors (FID)}

Example: Heath Consultants Model DP4

Principle of Operation: Measures electrical conductivity of a flame burning carbon compounds Sampling Method: Extractive (response time few seconds)

Sensitivity: Typically measures parts per million Advantages: Sensitive detection of low gas concentrations

Disadvantages: Sampling probe must be embedded within gas plume. Senses all combustion gases, not just natural gas.

Suitable for remote leak surveying: No

Suitable for mobile leak surveying: Only by traveling through leak plume

Suitable for airborne leak surveying: Only by traveling through leak plume.

\section{Optical Methane Detector (OMD) ${ }^{1}$}

Example: Heath Consultants

Principle of Operation: Absorption of Infrared Light by Methane

Sampling Method: Short Open Path or Extractive

Sensitivity: Typically measures parts per million 
Advantages: Sensitive detection of low gas concentrations; Open path probe eliminates sampling time lag of extractive sensors. Less sensitive to gases other than methane.

Disadvantages: Sampling probe must be embedded within or encompass gas plume

Suitable for remote leak surveying: No

Suitable for mobile leak surveying: Only by traveling through leak plume

Suitable for airborne leak surveying: Only by traveling through leak plume.

\section{Short-Path TDLAS}

Example: Boreal Laser

Principle of Operation: Differential Absorption of Infrared Light by Methane; single laser with temporal wavelength modulation

Sampling Method: Open Path or Extractive

Sensitivity: Typically measures $<1$ part per million

Advantages: Sensitive detection of low gas concentrations; Open path probe eliminates sampling time lag of extractive sensors, low power consumption. Senses only methane.

Disadvantages: Sampling probe must be embedded within or encompass gas plume

Suitable for remote leak surveying: No

Suitable for mobile leak surveying: Only by traveling through leak plume

Suitable for airborne leak surveying: Only by traveling through leak plume.

\section{RMLD/Stand-off TDLAS $S^{2,3}$}

Example: Physical Sciences Inc./Heath Consultants RMLD

Principle of Operation: Differential Absorption of Infrared Light by Methane; single laser with temporal wavelength modulation

Sampling Method: Detection of laser backscatter from topographic targets

Sensitivity: Measures path-integrated concentration, typical detection limit $\sim 5 \mathrm{ppm}-\mathrm{m}$

Advantages: Fast, sensitive detection of low gas concentrations; optical transceiver can be outside of gas plume, low power consumption. Senses only methane.

Disadvantages: New technology; plume must be encompassed between transceiver and topographic scattering surface; range limited to $100 \mathrm{ft}$

Suitable for remote leak surveying: Yes

Suitable for mobile leak surveying: Yes, under development

Suitable for airborne leak surveying: Current research project to extend range to $>10,000 \mathrm{ft}$.

\section{Active Gas Correlation Radiometry ${ }^{4}$}

Example: Ophir DUOthane

Principle of Operation: Differential Absorption of Infrared Light by Methane and Ethane;

Sampling Method: Detection of infrared backscatter from topographic targets

Sensitivity: Measures path-integrated concentration, typical detection limit $\sim 50 \mathrm{ppm}-\mathrm{m}$

Advantages: Detection of low gas concentrations; optical transceiver can be outside of gas

plume. Senses two components of natural gas.

Disadvantages: New technology; plume must be encompassed between transceiver and topographic scattering surface; range limited to $\sim 500 \mathrm{ft}$ 
Suitable for remote leak surveying: Yes Suitable for mobile leak surveying: Yes

Suitable for airborne leak surveying: Under development. Maximum altitude $\sim 500 \mathrm{ft}$.

\section{Differential Absorption LIDAR (DIAL) $)^{5,6}$}

Examples: ITT (fixed-wing aircraft with mapping and imaging), Lasen (helicopter), Gas Technology Institute (surface vehicle with imaging)

Principle of Operation: Differential Absorption of Infrared Light by Methane and Ethane; multiple lasers provide temporal wavelength modulation

Sampling Method: Detection of laser backscatter from topographic targets

Sensitivity: Measures path-integrated concentration, expected detection limit $\sim 5 \mathrm{ppm}-\mathrm{m}$ Advantages: Fast, sensitive detection of low gas concentrations; optical transceiver can be outside of gas plume

Disadvantages: New technology unproven for leak survey applications; plume must be encompassed between transceiver and topographic scattering surface; relatively high power consumption (compared to TDLAS).

Suitable for remote leak surveying: Yes

Suitable for mobile leak surveying: Yes, under development

Suitable for airborne leak surveying: Yes, under development. Maximum altitude $2000 \mathrm{ft}$.

\section{Passive Multi-wavelength Radiometry ${ }^{7}$}

Examples: En-Urga, PSI/AIRIS

Principle of Operation: Differential Absorption of Infrared Light by Methane;

Sampling Method: Passive detection of infrared light emitted by topographic surfaces. Tunable filters or several fixed filters provide differential absorption measurement.

Sensitivity: Measures path-integrated concentration, typical detection limit $\sim 500$ ppm-m

Advantages: No active illumination source, plume imaging possible

Disadvantages: New technology; plume must be encompassed between transceiver and topographic emitter; relatively poor sensitivity compared to active techniques; subject to cross-species interference; relatively slow; limited dynamic range; quantification difficult due to gas temperature effect

Suitable for remote leak surveying: Yes

Suitable for mobile leak surveying: Yes, under development

Suitable for airborne leak surveying: Possibly. Not currently in development. Maximum altitude $\sim 3000 \mathrm{ft}$.

\section{Summary}

The technologies currently available or under development for aerial leak surveying are summarized in Table 1. They fall into two broad categories: In-situ techniques requiring the aircraft to fly through a leak plume in order to detect the leak, and remote sensors based on optical standoff detection. The latter includes both active and passive optical sensors, all based on absorption of infrared light as it passes through the leak plume. Differential absorption, i.e. measurement of infrared absorption at two or more wavelengths, enables sensitive and selective 
detection of methane and, in some cases, ethane. The more-sensitive active techniques include the use of wavelength agile (i.e. tunable) lasers, or multiple lasers of fixed wavelength, or broadband (i.e. non-laser) infrared sources with narrow-band filters.

All of the current optical sensors have the potential for use in airborne surveys, and some have been tested in that mode, but they are limited to altitudes of about $2000 \mathrm{ft}$ agl. The amplified RMLD offers the potential to detect leak plumes having path-integrated concentrations $>1000$ ppm-m from altitudes up to $80,000 \mathrm{ft}$. From this altitude, entire cites could be continuously monitored from a UAV.

\begin{tabular}{|c|c|c|}
\hline \multicolumn{3}{|c|}{ Table 1 - Technology Summary } \\
\hline Technology & Pros & Cons \\
\hline \multicolumn{3}{|l|}{ In-situ Sensors } \\
\hline Flame Ionization Detector & $\begin{array}{l}\text { - Established Technology } \\
\text { - Sensitive Detection }\end{array}$ & $\begin{array}{l}\text { - Extractive } \\
\text { - Non-Specific }\end{array}$ \\
\hline Optical Methane Detector & $\begin{array}{l}\text { - Proven Technology } \\
\text { - Open-Path }\end{array}$ & - Must encompass plume \\
\hline Short-path TDLAS & $\begin{array}{l}\text { - Very sensitive and specific } \\
\text { - Open Path }\end{array}$ & - Must encompass plume \\
\hline \multicolumn{3}{|l|}{ Stand-off Sensors } \\
\hline \multicolumn{3}{|l|}{ Active } \\
\hline Stand-off TDLAS & $\begin{array}{l}\text { - Very sensitive and specific } \\
\text { - Remote detection }\end{array}$ & - Range limited to $100 \mathrm{ft}$ \\
\hline $\begin{array}{l}\text { Gas Correlation } \\
\text { Spectrometry }\end{array}$ & $\begin{array}{l}\text { - Specific to natural gas } \\
\text { - Remote detection }\end{array}$ & $\begin{array}{l}\text { - Less sensitive than TDLAS } \\
\text { - Range limited to } 500 \mathrm{ft}\end{array}$ \\
\hline $\begin{array}{l}\text { Differential Absorption } \\
\text { LIDAR }\end{array}$ & $\begin{array}{l}\text { - Very sensitive and specific } \\
\text { - Remote Detection Range } ~ \\
2000 \mathrm{ft}\end{array}$ & $\begin{array}{l}\text { - Complex Operation } \\
\text { - Unproven }\end{array}$ \\
\hline \multicolumn{3}{|l|}{ Passive } \\
\hline $\begin{array}{l}\text { Multi-Wavelength } \\
\text { Radiometry }\end{array}$ & $\begin{array}{l}\text { - No active illumination } \\
\text { source } \\
\text { - Imaging possible }\end{array}$ & $\begin{array}{l}\text { - Poor sensitivity and } \\
\text { specificity } \\
\text { - Limited dynamic range } \\
\text { - Difficult to quantify }\end{array}$ \\
\hline
\end{tabular}

\section{References}

1. H. Lessure and K. Kothari, "New Handheld Optical Portable Methane Detector," in Proceedings of Natural Gas Technologies II: Ingenuity \& Innovation, Gas Technology Institute Publication GTI-04/0012, Phoenix, AZ, February 2004.

2. A.G. Fabiano, B.D. Green, P. Wehnert, and S. Chancey, "Remote Methane Leak Detector; Advanced Development and Field Tests," in Proceedings of Natural Gas Technologies II: Ingenuity \& Innovation, Gas Technology Institute Publication GTI-04/0012, Phoenix, AZ, February 2004. 
3. M.B. Frish, B.D. Green, F. Scire-Scappuzzo, P. Cataldi, A. Burbo, M. Laderer, and G. Midgley, "Mobile remote Methane Leak Detection Demonstration," in Proceedings of Natural Gas Technologies II: Ingenuity \& Innovation, Gas Technology Institute Publication GTI-04/0012, Phoenix, AZ, February 2004.

4. J. Myers and L. Spaet, "Airborne, Optical Remote Sensing of Methane and Ethane for Natural Gas Pipeline Leak Detection," in Proceedings of Natural Gas Technologies II: Ingenuity \& Innovation, Gas Technology Institute Publication GTI-04/0012, Phoenix, AZ, February 2004.

5. R. Farmer, T. McRae, and K. Kothari, "Laser-based Remote Sensing of Gas Distribution Leaks," in Proceedings of Natural Gas Technologies II: Ingenuity \& Innovation, Gas Technology Institute Publication GTI-04/0012, Phoenix, AZ, February 2004.

6. D. Brake, M. Clayton, and S. Stearns, "Advanced Airborne Natural Gas Leak Detection System Overview," in Proceedings of Natural Gas Technologies II: Ingenuity \& Innovation, Gas Technology Institute Publication GTI-04/0012, Phoenix, AZ, February 2004.

7. B.R. Cosofret, W.J. Marinelli, T. Ustun, C.M. Gittins, M.T. Boies, M.F. Hinds, D.C. Rossi, C. Coxe, and S. Chang, "Passive Infrared Imaging Sensor for Standoff Detection of Methane Leaks," presented at SPIE Optics East Chemical and Biological Standoff Detection II (Philadelphia, PA), October 2004. 
PSI-1454

\title{
Appendix C
}

\section{Sensor Specification \\ (Version 1)}

\section{High-Altitude, Aerial Natural Gas Leak Detection System}

Contract No. DE-FC26-04NT42268

\author{
Prepared by: \\ Mickey B. Frish \\ Physical Sciences Inc. \\ 20 New England Business Center \\ Andover, MA 01810-1077 \\ Prepared for: \\ National Energy Technology Laboratory \\ U.S. Department of Energy \\ 3610 Collins Ferry Road, P.O. Box 880 \\ Morgantown, WV 26507-0880
}

January 2005

\section{ACKNOWLEDGEMENT STATEMENT:}

This Technology Status Assessment was prepared with the support of the U.S. Department of Energy, under Award No. DE-FC26-04NT42268. However, any opinions, findings, conclusions, or recommendations expressed herein are those of the author(s) and do not necessarily reflect the view of the DOE.

\section{DISCLAIMER}

This report was prepared as an account of work sponsored by an agency of the United States Government. Neither the United States Government nor any agency thereof, nor any of their employees, makes any warranty, express or implied, or assumes any legal liability or responsibility for the accuracy, completeness, or usefulness of any information, apparatus, product, or process disclosed, or represents that its use would not infringe privately owned rights. Reference herein to any specific commercial product, process, or service by trade name, trademark, manufacturer, or otherwise does not necessarily constitute or imply its endorsement, recommendation, or favoring by the United States Government or any agency thereof. The views and opinions of authors expressed herein do not necessarily state or reflect those of the United States Government or any agency thereof. 


\section{System}

\section{Configuration:}

6 component system:

(1) 19" rack-mounted laser control and signal processing electronics box (controller), (2) 19" rack-mounted fiber amplifier (EDFA), (3) optical transceiver (telescope),

(4) digital video tracking camera, (5) GPS with data output,

(6) data-recording computer,

\section{Performance:}

General:

Able to sense natural gas leak plumes having minimum pathintegrated methane concentrations of $1000 \mathrm{ppm}-\mathrm{m}$. This is expected to be sufficient to detect the presence of a leak from a high-capacity transmission line.

Measurement technique:

Tunable diode laser absorption spectroscopy (TDLAS) using wavelength modulation spectroscopy (WMS) noise-reduction technique

Detection selectivity:

Methane. No sensitivity to any other member of the paraffin series, or vehicle exhaust fumes (gasoline and diesel), or natural atmospheric constituents $\left(\mathrm{H}_{2} \mathrm{O}, \mathrm{O}_{2}, \mathrm{CO}_{2}\right)$

Measurement range:

0 ppm-m to 100,000 ppm-m

Maximum target distance: 3000 meters $(9,900$ feet)

Min. operational target distance: 50 meters (165 feet)

Min. eye-safe operational distance: 24 meters ( 80 feet)

Detection area:

$10 \mathrm{~m}$ at $3000 \mathrm{~m}$ altitude $(0.3 \mathrm{~m}$ at $100 \mathrm{~m}$ altitude $)$

Sensitivity:

Accuracy:

1000 ppm-m at all altitudes

Noise-equivalent detection limit:

No false negatives and minimal false positives

Data sample rate:

100 ppm-m

Ground scanning rate:

10 times per second.

Fixed to air speed. Nominally $50 \mathrm{~m} / \mathrm{s}$ ( $5.0 \mathrm{~m}$ per datapoint) at $112 \mathrm{miles} / \mathrm{hr}$

Pointing/scan control:

None. Sensor points directly down, fixed to aircraft

Pointing stability: $60 \mathrm{~m}$ due to plane roll jitter of $1.0^{\circ}$

\section{Power Requirements:}

Controller:

EDFA:

6.0-8.4 VDC at $<5 \mathrm{~W}$ (or Li ion battery ( $\sim 8 \mathrm{hr}$ duration)) $28 \mathrm{VDC}$ at $<130 \mathrm{~W}$ 


\section{Safety and Regulatory:}

IR sensor laser category: $\quad$ Class IV non-Eye Safe*, $5 \mathrm{~W}$ max (CDRH, ANSI and IEC) OSHA Infrared "B" region: Danger to eye lens and cornea

IR sensor laser output:

$5 \mathrm{~W} \max \left(2 \mathrm{~W} / \mathrm{cm}^{2}\right.$ at exit aperture $)$

*Operationally eye-safe at ground level $\left(\sim 6 \mu \mathrm{W} / \mathrm{cm}^{2}\right.$ at

$3000 \mathrm{~m}$ sensor height, $\sim 6 \mathrm{~mW} / \mathrm{cm}^{2}$ at $\left.100 \mathrm{~m}\right)$

Standard: Class IV ANSI Intraocular Maximum Permissible Exposure (Doc. Z136.1-1993) $=100 \mathrm{~mW} / \mathrm{cm}^{2}$

\section{Environmental (operational):}

Temperature:

Components in plane interior:

0 to $+35 \mathrm{C}(+32$ to $+95 \mathrm{~F})$

Controller, EDFA, computer

Exterior components:

-10 to $+50 \mathrm{C}(+14$ to $+122 \mathrm{~F})$

Transciever, video camera

Humidity:

5 to $95 \% \mathrm{RH}$, non-condensing

Vibration (isolation):

Rubber isolation bushings in rack-mount components.

Rubber isolation supports on transceiver/camera

\section{Material / Housing:}

Transceiver:

Black anodized aluminum

Controller, EDFA, computer:

19 " rack-mount casings

\section{Alarms:}

Concentration:

None

Faults:

Silent alarm, fault record.

Field Verification:

Built-in wavelength test on ambient methane or sealed cell.

\section{Cabling:}

Optical fibers:

PVC-clad SM FC/APC fiber patchcord $(0.5 \mathrm{~m})$ between controller and EDFA. Steel monocoil-clad FC/APC patchcord $(1 \mathrm{~m})$ between EDFA and transceiver

Electrical: DB-9 serial between controller and computer. 4-wire braided cable with PVC jacket and weathertight connectors between controller and transceiver.

\section{PC Interface:}

LabWindows graphical interface and datalogger.. 


\section{Transceiver}

\section{Configuration:}

Reflective or catadioptric 10" diameter telescope with secondary focusing to image onto $1.5 \mathrm{~mm}$ InGaAs photodiode. Beam launch (fiber to 1"dia. OAP mirror) and videocamera are attached to and aligned with side of telescope tube.

\section{Physical:}

Dimensions:

12" diameter $\mathrm{x} 48$ " long telescope tube

Tube weight:

30 lbs.

With mounts, camera, launch: $50 \mathrm{lbs}$.

Laser Beam (IR): $\quad$ Continuously on with instrument power.

Diameter: $\quad 10 \mathrm{~m}$ dia at $3000 \mathrm{~m}$

$18 \mathrm{~mm}$ dia at launch

Output power:

$5 \mathrm{~W} \max$

Output power density:

$6 \mu \mathrm{W} / \mathrm{cm}^{2}$ at $3000 \mathrm{~m}(10 \mathrm{~m}$ spot) (ANSI eye-safe) $100 \mathrm{~mW} / \mathrm{cm}^{2}$ at $24 \mathrm{~m}$ (8cm spot) (ANSI eye-safe limit)

$2 \mathrm{~W} / \mathrm{cm}^{2}$ at launch mirror (1.8cm spot) (non-ANSI eye-safe) Standard: Class IV ANSI Intraocular Maximum Permissible Exposure (Doc. Z136.1-1993) $=100 \mathrm{~mW} / \mathrm{cm}^{2}$

Wavelength:

Nominally 1.618 micrometers.

Pointing Stability:

At best, $20 \mathrm{mrad}\left(1^{\circ}\right)(60 \mathrm{~m}$ on ground from $3000 \mathrm{~m}$ height $)$ due to plane roll jitter 


\section{Camera}

\section{Configuration:}

Monochrome CCD camera ( $\sim 1 / 2$ " format) with video output, combined with manual telephoto zoom lens.

\section{Physical:}

Size:

Weight:

3" dia. x 10" long

Less than 2 lbs.

\section{Performance:}

Field of view:

CCD format:

$75 \mathrm{~m}$ to $535 \mathrm{~m}(7 \mathrm{x})$ at $3000 \mathrm{~m}$ altitude

Spatial resolution: $\sim 500$ pixels square over $5 \mathrm{~mm}$ square

$0.15 \mathrm{~m}$ to $1.1 \mathrm{~m}$ at $3000 \mathrm{~m}$ altitude 


\section{Physical:}

\section{Controller}

Size:

Weight:

Power Supply:

Power Consumption:

On-board TDL Laser (IR):

Output power:

Output power density:

Wavelength:

Output Linewidth:

Laser warm up:

User interface / controls:

Optical Fiber Port:

Data Port: $\quad$ RS 232 serial port, DB - 9 male, DCE.

Non-Volatile Memory:

EEPROM for user's last setup, serial number and calibration.

Data Output for Logging:
17.5 x $17.5 "$ x $3 ", 19$ " rack-mount

Less than $10 \mathrm{lbs}$.

$28 \mathrm{~V} \rightarrow 8 \mathrm{~V}$ DC converter, or rechargeable Li ion battery

$<5 \mathrm{~W}$

Continuously on with instrument power. Fiber-coupled to instrument bulkhead fitting.

$25 \mathrm{~mW} \max$

$1.5 \mathrm{~W} / \mathrm{cm}^{2}$ at fiber port (1.5mm spot) (non-ANSI eye-safe)

Standard: Class IV ANSI Intraocular Maximum Permissible Exposure (Doc. Z136.1-1993) $=100 \mathrm{~mW} / \mathrm{cm}^{2}$

Nominally 1.618 micrometers.

$\sim 100 \mathrm{MHz}$

10 seconds

Power on / off switch.

RS 232 serial port.

Singlemode FC/APC
Default output format when in gas detection mode.

Serial number

Error codes

Analyte concentration (ppm-m)

Preamp DC level

F1 value

F2 orthogonal values

TDL modulation level

TDL drive current

TDL temperature 


\section{Fiber Laser Amplifer}

Type:

\section{Physical:}

Size:

Weight:

\section{Optical Specifications:}

Operating wavelength:

Input power

Output power

Input Linewidth:

Output Linewidth:

Spatial Field:

Output power stability:

Optical isolation:

\section{Power Supply:}

Power Consumption:

User interface / controls:
Benchtop erbium-doped fiber amplifier (EDFA) (Keopsys), with auomatic current control (ACC), alarms, \& status indicators

17.5 " 17.5 " $13.5 ", 19$ " rack-mount

Less than $10 \mathrm{lbs}$.

$1616-1618 \mathrm{~nm}$

$10 \mathrm{~mW}$ to $15 \mathrm{~mW}(\mathrm{CW})$

$5 \mathrm{~W}$ CW over operating wavelength range

$>1 \mathrm{MHz}$

$<1 \mathrm{GHz}$

$\mathrm{TEM}_{00}$

$<3 \%$ variation peak to peak at fixed temperature input isolator $(>20 \mathrm{~dB})$

output isolator $(>20 \mathrm{~dB})$

$28 \mathrm{VDC}$

$<130 \mathrm{~W}$

Power on / off keyswitch.

RS 232 / GPIB interface 


\section{Proposed Component Layout}

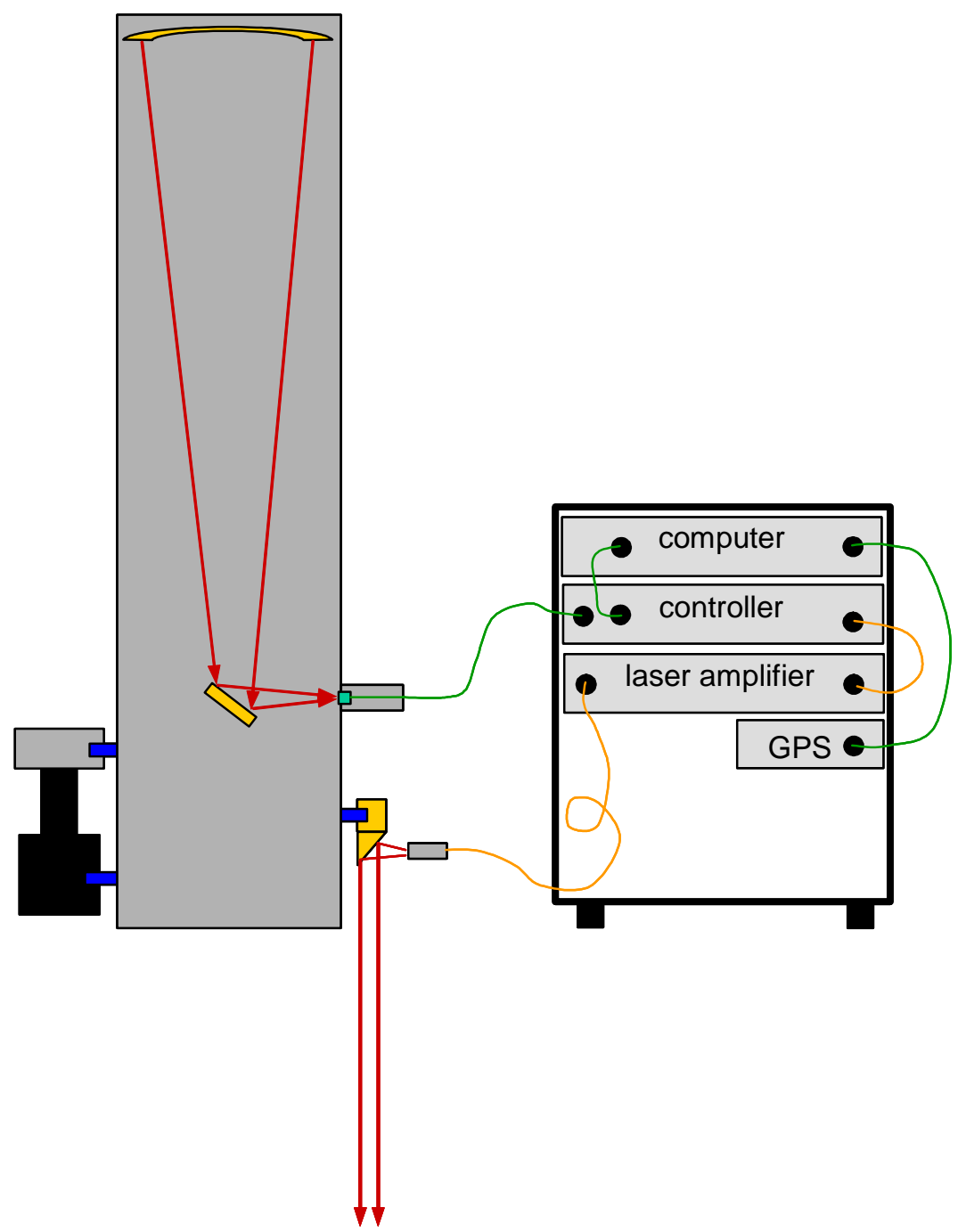

Figure 1. Airborne natural gas leak sensor component schematic. 


\section{Proposed Connectivity Layout (Functional, not actual)}

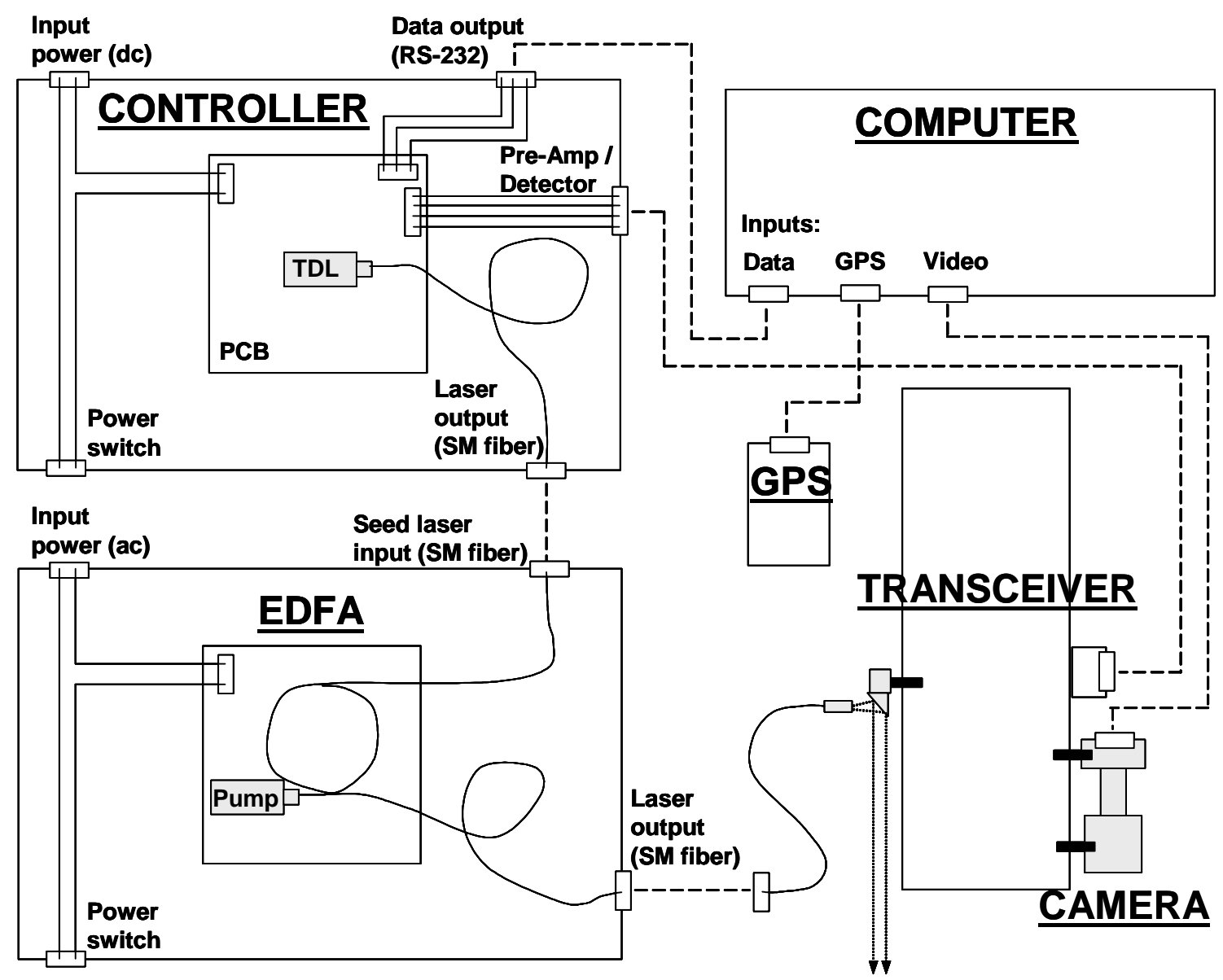

Figure 2. Airborne natural gas leak sensor cabling schematic. 


\section{Operating Procedure}

\section{$\underline{\text { Pre-flight }}$}

Alignment:

Tuning/calibration:

Installation:
In $\sim 1000 \mathrm{ft}$ open field, orient telescope horizontally and send visible alignment laser through beam launch fiber. Center spot on target at $1000 \mathrm{ft}$ distance with OAP ultrafine adjusters. Image through ocular placed in telescope output. Replace visible beam with IR beam and ocular with InGaAs detector package. Tweak alignment to maximize received signal. With video output from camera sent to a monitor, align camera with ultrafine adjusters to center image on target location. Lock all adjusters.

With signal from target at $1000 \mathrm{ft}$, tune laser wavelength with dc laser power scan on the background $\mathrm{CH}_{4}(\sim 1200 \mathrm{ppm}-\mathrm{m})$ or leak a calibration gas into telescope tube for stronger signal. Calculate and set calibration constant based on this background or purge gas.

In parked plane (tilted?) install electronics rack and video monitor. Split monitor output to small monitor for pilot. Install telescope in suspension device and lock down with a clamping support structure. Start plane, send power to system, boot up, and turn on laser/EDFA. Check performance.

\section{$\underline{\text { In-flight }}$}

Alignment:

Operate sensor:

Release telescope support structure and adjust telescope suspension, if needed, to orient field of view normal to plane.

Align plane with target (pipeline) via video monitor. Record sensor signals, GPS data, and video output $(30 \mathrm{~Hz}$ reduced to $10 \mathrm{~Hz}$, stored to DVD). 
PSI-1454

\title{
Appendix D
}

\section{Experimental Prototype (EP) Flight Test Plan \\ (Version 1)}

\section{High-Altitude, Aerial Natural Gas Leak Detection System}

Contract No. DE-FC26-04NT42268

\author{
Prepared by: \\ Mickey B. Frish \\ Physical Sciences Inc. \\ 20 New England Business Center \\ Andover, MA 01810-1077 \\ Prepared for: \\ National Energy Technology Laboratory \\ U.S. Department of Energy \\ 3610 Collins Ferry Road, P.O. Box 880 \\ Morgantown, WV 26507-0880
}

September 2006

ACKNOWLEDGEMENT STATEMENT:

This Technology Status Assessment was prepared with the support of the U.S. Department of Energy, under Award No. DE-FC26-04NT42268. However, any opinions, findings, conclusions, or recommendations expressed herein are those of the author(s) and do not necessarily reflect the view of the DOE.

\section{DISCLAIMER}

This report was prepared as an account of work sponsored by an agency of the United States Government. Neither the United States Government nor any agency thereof, nor any of their employees, makes any warranty, express or implied, or assumes any legal liability or responsibility for the accuracy, completeness, or usefulness of any information, apparatus, product, or process disclosed, or represents that its use would not infringe privately owned rights. Reference herein to any specific commercial product, process, or service by trade name, trademark, manufacturer, or otherwise does not necessarily constitute or imply its endorsement, recommendation, or favoring by the United States Government or any agency thereof. The views and opinions of authors expressed herein do not necessarily state or reflect those of the United States Government or any agency thereof. 


\section{Purpose and Overview}

This document describes the test plan for the experimental flight testing of the airborne remote methane leak detector (aRMLD). The aRMLD is envisioned as a future product, based on the currently-available handheld RMLD, to be installed on aircraft that will fly over natural gas pipelines and survey for natural gas leaks. The data storage function of the software interface will enable matching of detected leak signals with geographical location. The interface will also enable the acquisition of data that will assist in creating a clear definition of the performance required for leak detection strategy, in finding effective methods of survey, and in the assessment of potential barriers to acceptance.

The EP configuration utilizes an enhanced (over RMLD) interface, with a laptop computer for data display and storage, that incorporates input from a video camera that supplies an image of the scene that the aRMLD is surveying.

The entire operation is allocated 5 working days, 2 for installation and ground testing, 2 days for flight tests, and 1 for contingency.

\section{Installation / Ground Tests:}

\begin{tabular}{|c|c|}
\hline Air Platform: & Cessna 207 \\
\hline Contractor: & Gamm Air, Inc. (Pottstown, PA) (www.gammair.com) \\
\hline Pilot: & Len Subik \\
\hline \multicolumn{2}{|l|}{ Payload Specifications: } \\
\hline Maximum payload: & xxxxx lbs \\
\hline Surveying port: & 22 " diameter open hole (7" interior floor to external skin) \\
\hline Survey Equip. Headspace: & $46 "$ \\
\hline
\end{tabular}
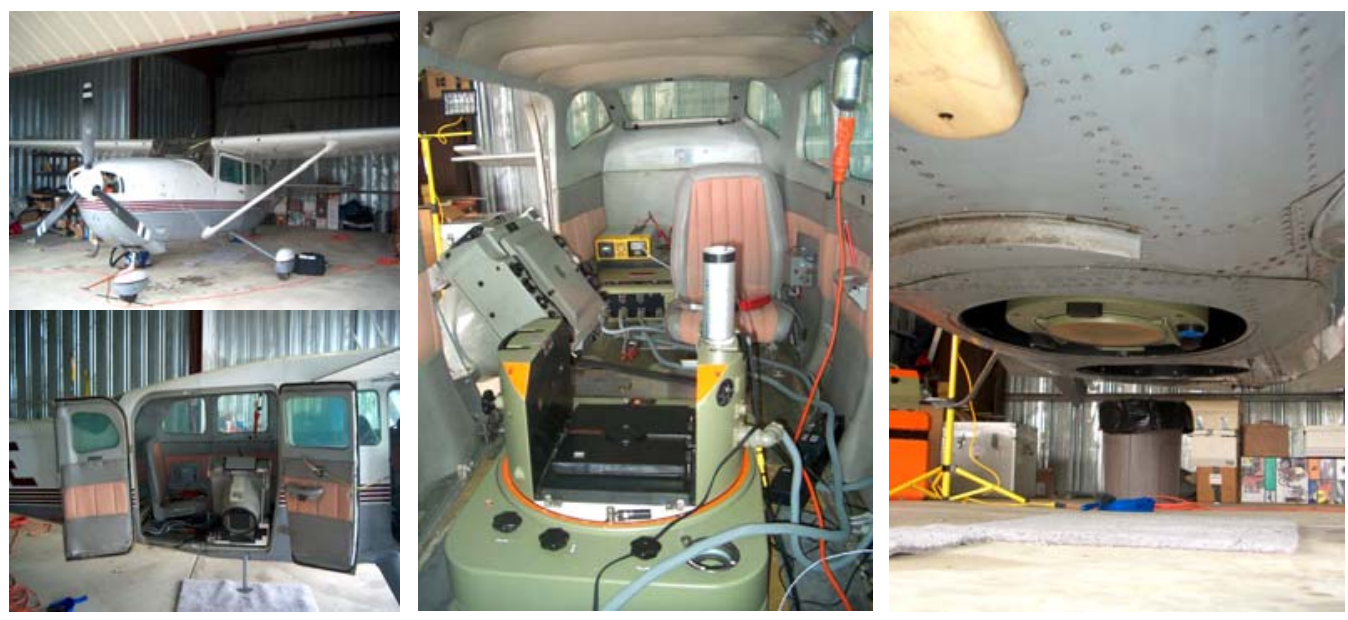

Figure 1. Gamm Air's Cessna 207, cabin with photographic equipment installed, and exterior view of camera port. 


\section{Sensor Hardware:}

Transceiver:

Laser Amplifier:

Laser / Signal Controller:

Computer:
12 inch dia. $x$ 48" Newtonian telescope (10 inch $f / 4.7$ primary) with side-mounted laser beam launch and monochrome video camera.

Keopsys 5W Erbium-doped Fiber Amplifier (EDFA) (28Vdc, 6A)

PSI single-PCB laser controller and WMS signal processor (battery-powered)

Windows-based laptop with LabWindows graphical interface and datalogger. (battery-powered)

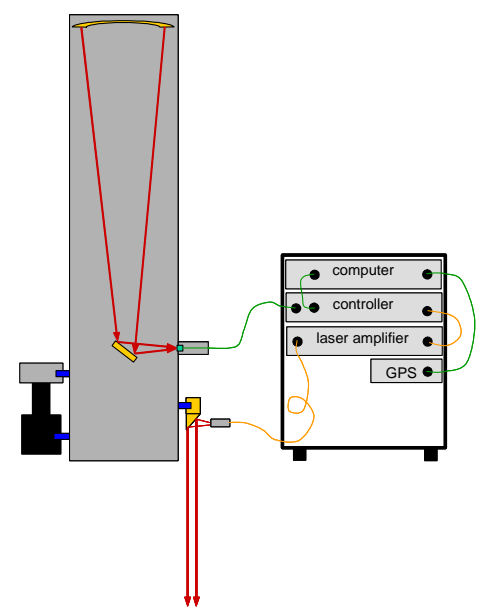

Figure 2. Mobile natural gas leak sensor computer-interfaced component schematic. (shown with optional GPS input). 

and Controller positioned near survey hole, strapped down with cargo straps. Transceiver suspended in hole with 4-legged frame with vibration-damping mounts. (see Figure 3)
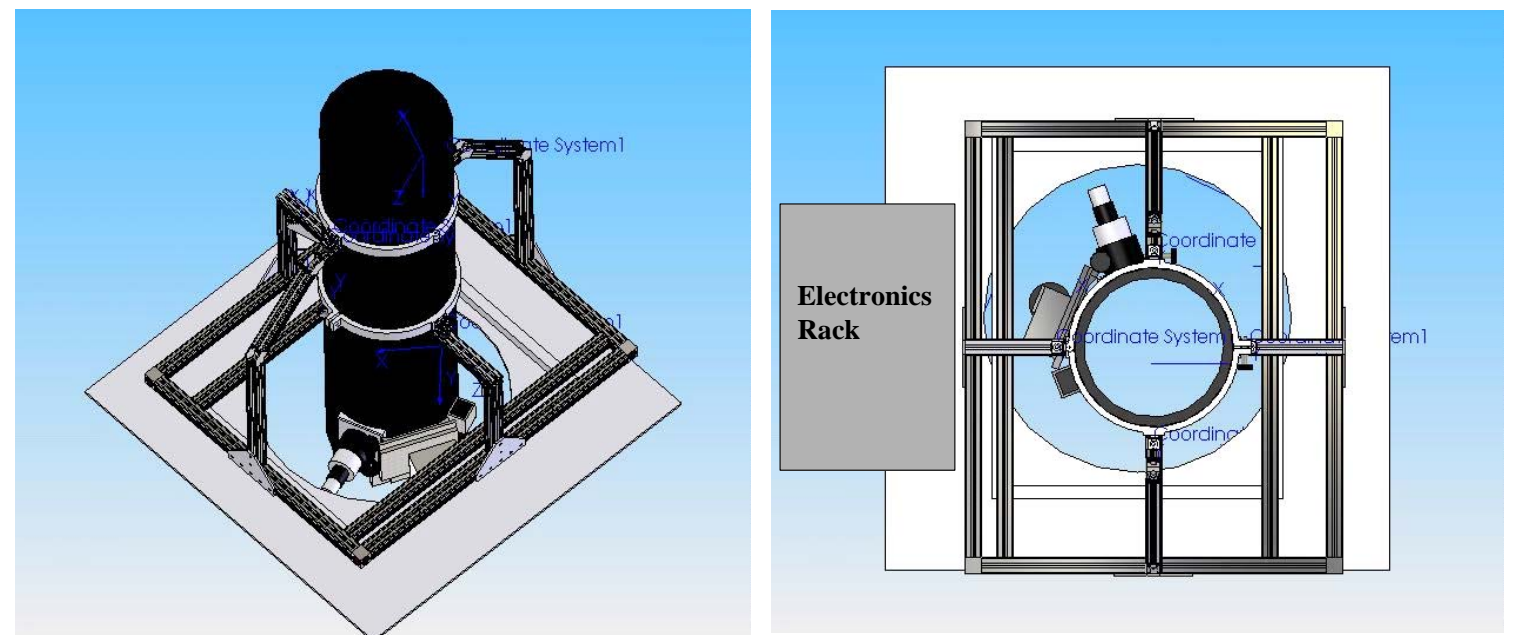

Figure 3. Airborne remote methane leak detector mounting apparatus drawing.

\section{Ground Tests:}

Operability:

Alignment:

Noise from air platform:

\section{Flight Tests:}

Ground support:

Leak Rates:
Test all equipment for operability, especially high power draw EDFA.

Transceiver will be pre-aligned. Fine alignment after installation is not possible. Check for potential misalignment during installation with a preconstructed laser beam-to-telescope f.o.v. template.

Record signals with engine off (airport electrical supply) and with engine on (aircraft electrical supply). Also determine potential for leaving EDFA running (warming up) during switchover from one supply to the other.

A person or team will be required on the ground at the predetermined leak site to control the regulation of the natural gas leak. A wind sock will be installed at the site as a leak location marker and wind indicator.

Leaks will be generated at one of two flow rates, nominally distinguished 'high' or 'low'. The high flow rate will be $\sim 5000$ SCFH and the low flow rate will be $\sim 1000 \mathrm{SCFH}$. If the leak is to be generated by gas cylinders, multiple cylinders will need to be utilized in either case. A $300 \mathrm{ft}^{3}$ cylinder will last only $18 \mathrm{~min}$ at the low flow rate and $3.6 \mathrm{~min}$ at the high flow rate. In order to develop a realistic leak plume, the leak should begin at least $15 \mathrm{~min}$ 
Flight Test 1:

Flight Test 2:

\section{Software:}

Input Signals:

WMS circuit board:

Camera:

Data Processing: before the aircraft flies over and the sensor performs its survey. Coordination with the airborne survey will be made by turning the leak on at a prescribed time, and similarly timing the first flyover to occur at a prescribed time.

The surveys on this test day will utilize the high leak flow rate. The test will be broken into 4 segments, delineating 4 different altitudes: $500 \mathrm{~m}, 1000 \mathrm{~m}, 2000 \mathrm{~m}$, and $3000 \mathrm{~m}$. At each altitude, there will be 4 passes over the leak site; 1 back $\&$ forth pass parallel to the ground wind direction, and 1 back $\&$ forth pass orthogonal to the ground wind direction. Approximately 15 minutes will be required at each altitude.

The surveys on this test day will utilize the low flow rate. Depending on observed signal responsivity to the high flow rate from the day before, this flow rate may be adjusted up or down from $1000 \mathrm{SCFH}$ to better record informative data. Again, the $1 \mathrm{hr}$ test will be broken into surveys at 4 altitudes and 8 passes at each altitude, like flight test 1 .

$10 \mathrm{~Hz}$ digital data that include:

Serial number.

Error codes.

Detected gas (PPM-M)

Battery level.

Preamp DC level.

TDL modulation level.

TDL drive current.

TDL temperature.

F1 value.

F2i, F2q, and F2 values.

$30 \mathrm{fps}$ analog $\left(1 \mathrm{~V}_{\mathrm{p}-\mathrm{p}}\right)$ video data

All input signals provided by the WMS board are to be recorded at $10 \mathrm{~Hz}$. These data will be processed after flight to evaluate leak detection success. Real-time processing algorithms will be utilized, but it is likely that temporal drifts of instrumental offsets will complicate real-time analysis.

Data analysis will identify the methane leak as a rapid change in methane signal compared to background. Each of the return-power normalized phase-orthogonal components of the F2 signal (i.e. $\mathrm{F} 2 \mathrm{i} / \mathrm{F} 1$ and $\mathrm{F} 2 \mathrm{q} / \mathrm{F} 1$ ) will be analyzed, thus minimizing the effect of phase drift on the offset. 
Data Display:

Data Storage:
LabWindows graphical interface. Includes:

Scrolling graphical displays (with adjustable axes):

Gas concentration

Received laser power (1f)

Absorption signal (2f)

Total light received at detector $(\mathrm{dc})$

Laser temperature

Numerical readouts:

Laser operating parameters: $\mathrm{I}_{\mathrm{dc}}, \mathrm{I}_{\mathrm{ac}}, \mathrm{T}_{\text {laser }}$ (useradjustable)

Battery voltage

Errors / warnings

Video rate updating image from camera

Toggle switch control in LabWindows graphical interface. Data stored:

All WMS input signals $(10 \mathrm{~Hz})$

Video image file (Compressed, if possible) (Userselectable storage rate (incl 'off'). Default 3Hz.) (Time-stamped to sub-second accuracy)

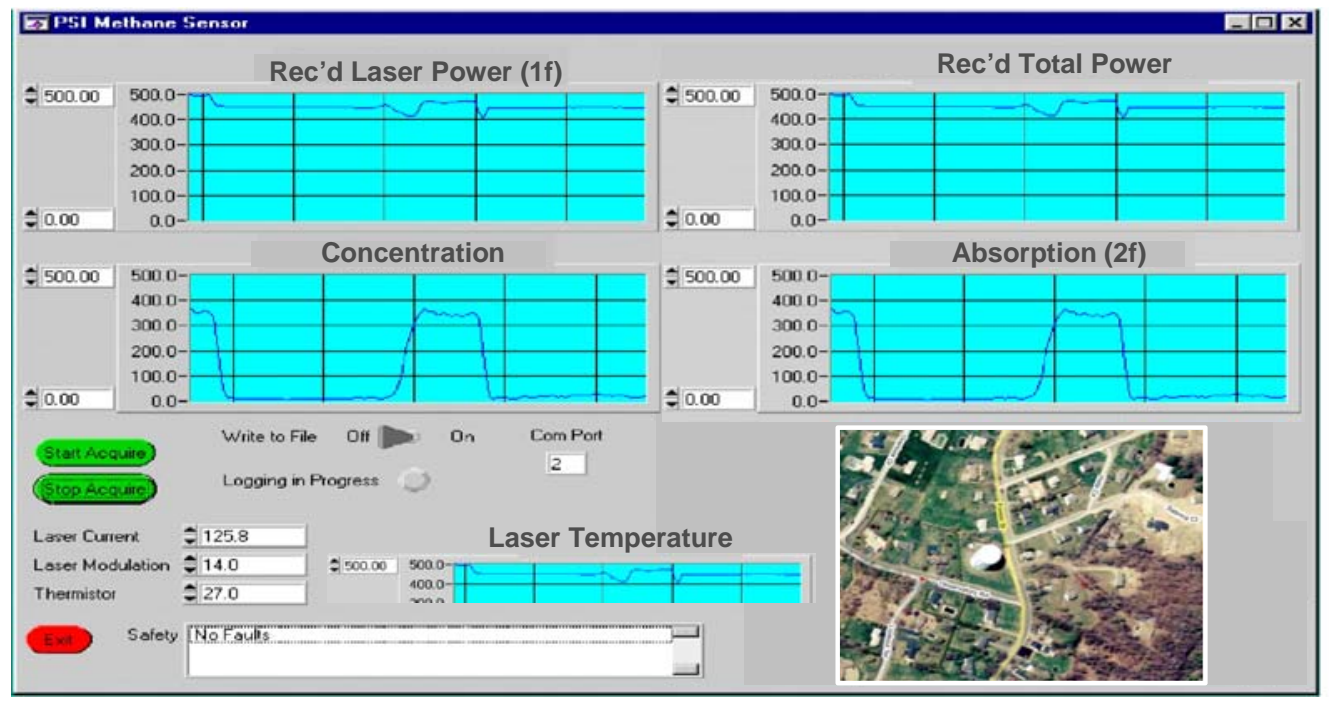

Figure 4. Example computer control software user interface. 